Abschlussbericht zur

\title{
Evaluierung von TecNet - Netzwerk für Markt- und Technologieinformationen der Innovationsagentur
}

Wien, 31. Juli 2002

\author{
Ansprechpartner \\ Andreas Fier/ Klaus Zinöcker \\ Institut für Technologie- und Regionalpolitik \\ JOANNEUM RESEARCH \\ Forschungsgesellschaft $\mathrm{mbH}$ \\ Wiedner Hauptstr. 76 \\ A-1040 Wien \\ Telefon: +43 (1)581 7520 - 2811 \\ Fax: $\quad+43(1) 5817520-2820$ \\ Email: andreas.fier@joanneum.at \\ klaus.zinoecker@joanneum.at
}


ABBILDUNGSVERZEICHNIS ........................................................................................5

TABELLENVERZEICHNIS ................................................................................................5

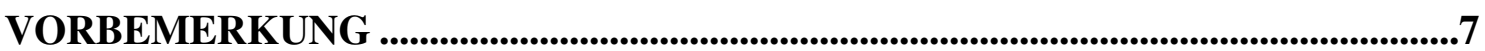

1 ZUSAMMENFASSUNG UND BEURTEILUNG ...................................................9

2 WIRTSCHAFTSPOLITISCHER HANDLUNGSBEDARF .................................. 21

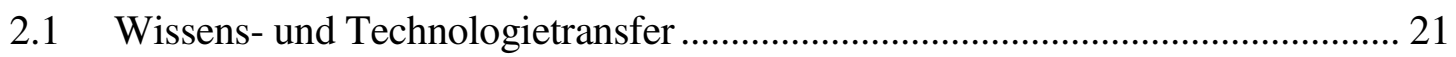

2.2 Ausgangslage und Handlungsbedarf in Österreich................................................ 22

2.3 Massnahmen des BMWA und die Entstehung des ,TecNet“ "............................ 23

3 TECNET - NETZWERK DER INNOVATIONSAGENTUR ................................ 25

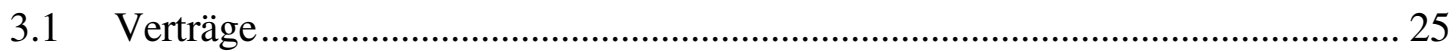

3.2 Errichtung und Organisationsstruktur ……....................................................... 25

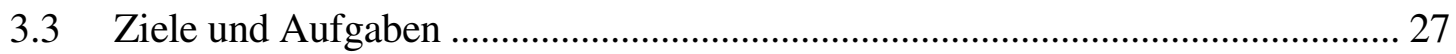

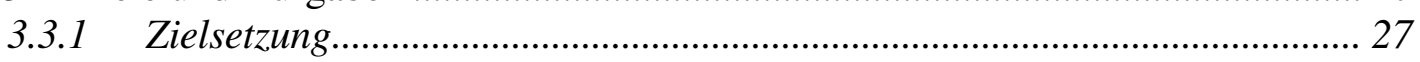

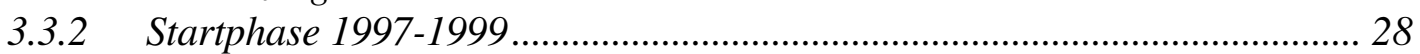

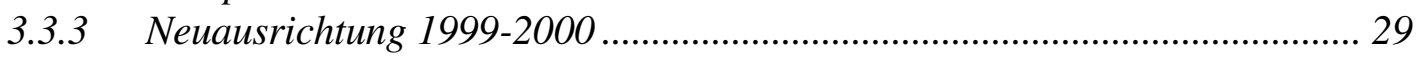

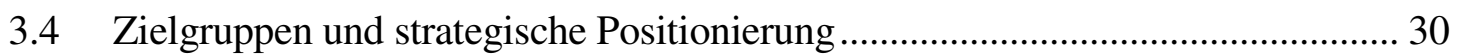

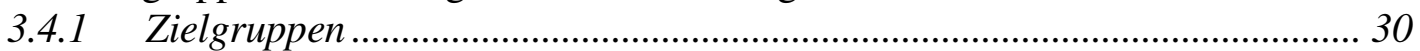

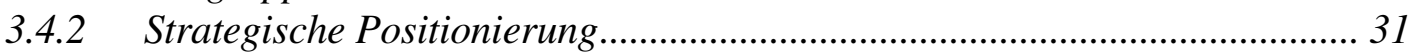

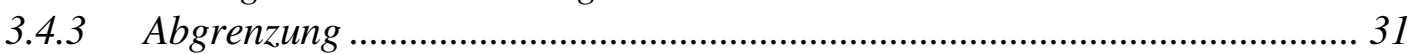

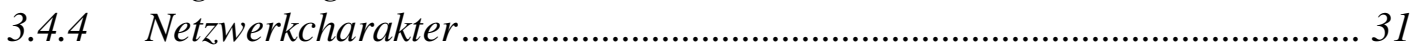

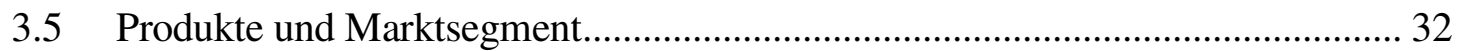

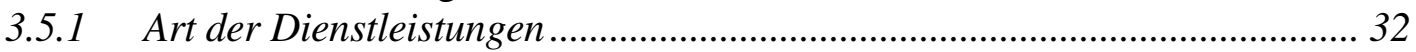

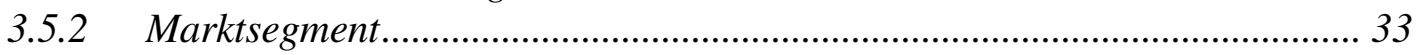

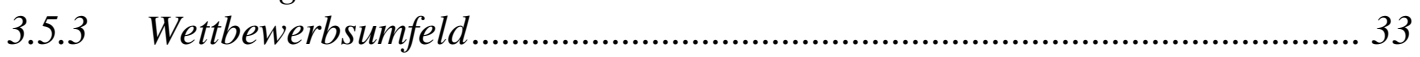

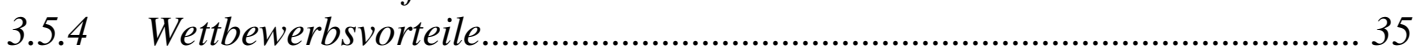

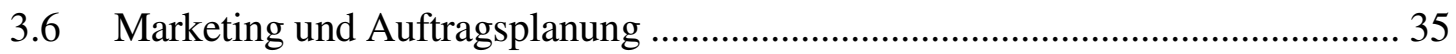

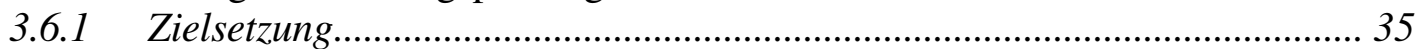

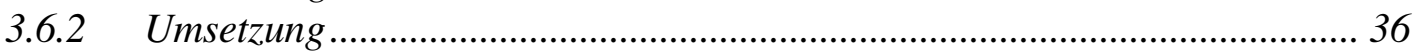

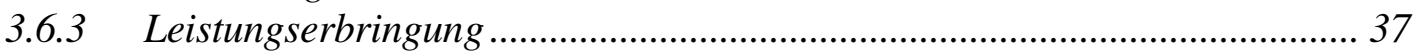

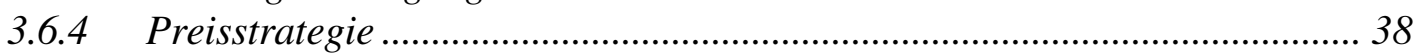

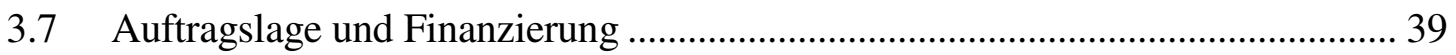

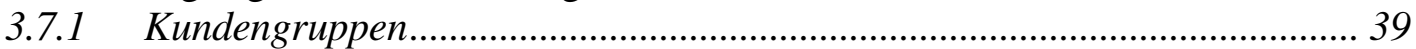

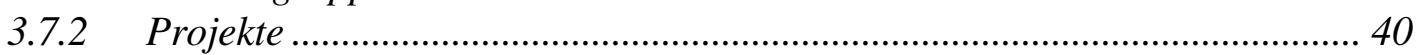

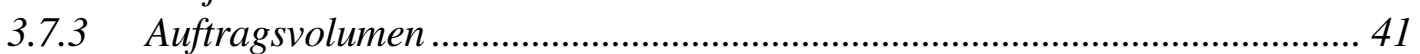

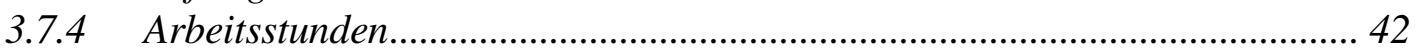

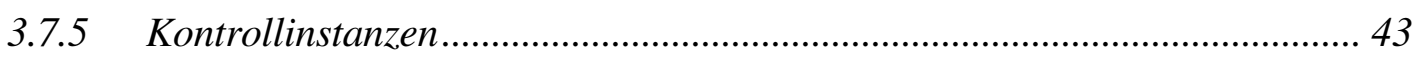

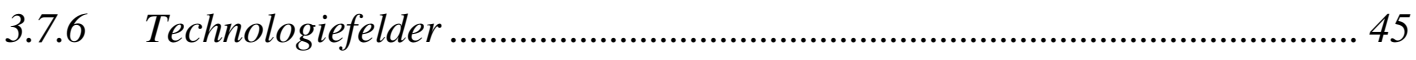




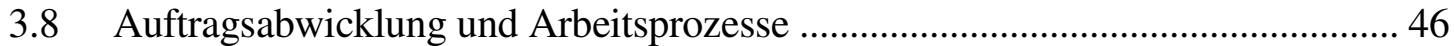

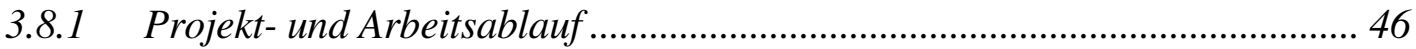

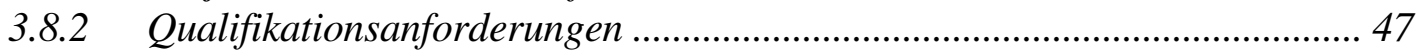

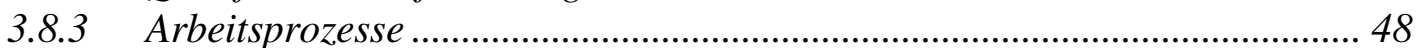

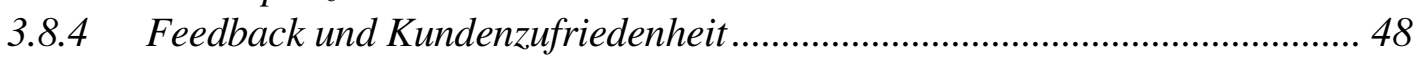

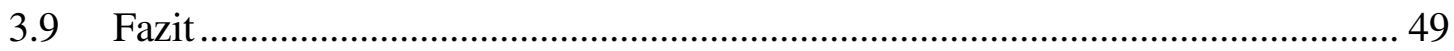

4 POTENZIALANALYSE UND UNTERNEHMENSBEFRAGUNG.................... 57

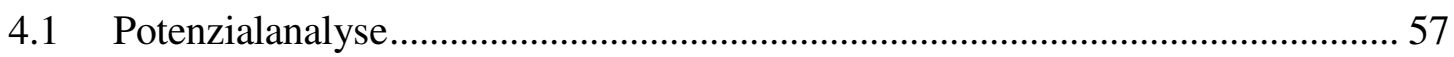

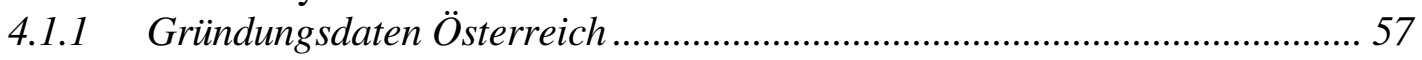

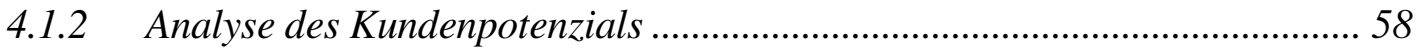

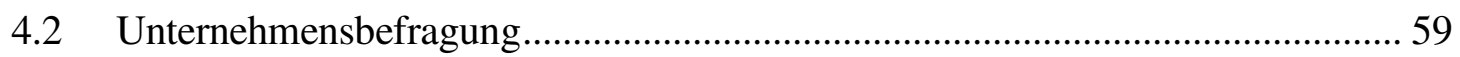

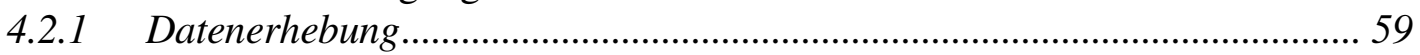

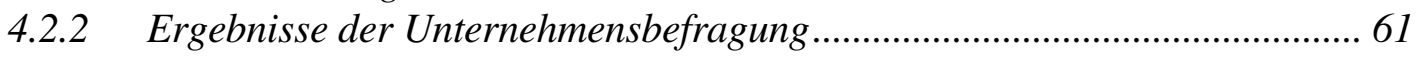

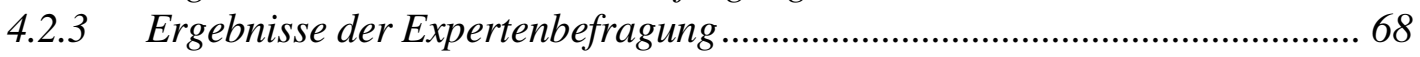

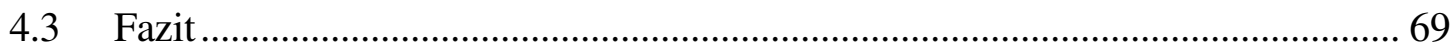

5 PROGRAMM- UND INSTITUTIONENVERGLEICH........................................... 73

5.1 Der Forschungsförderungsfonds für die Gewerbliche Wirtschaft (FFF)............ 73

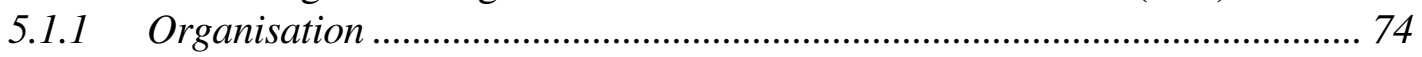

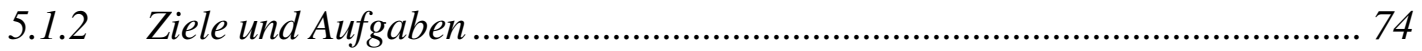

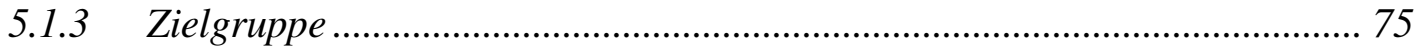

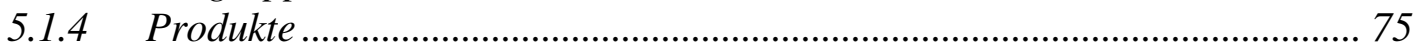

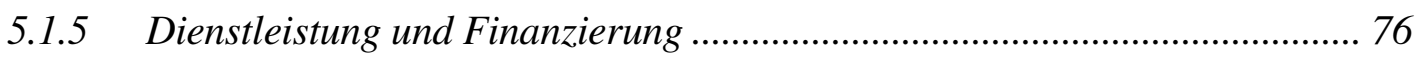

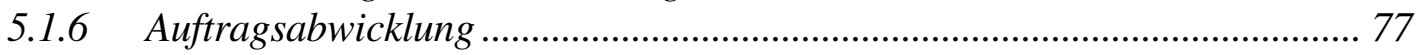

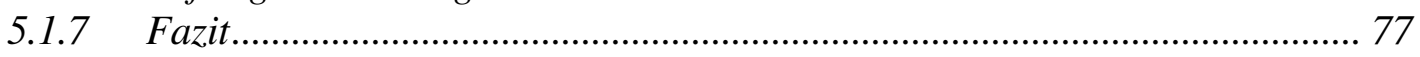

5.2 Das deutsche Fachinformationszentrum Karlsruhe (FIZ) .................................. 78

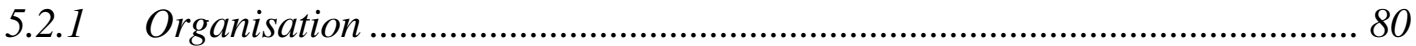

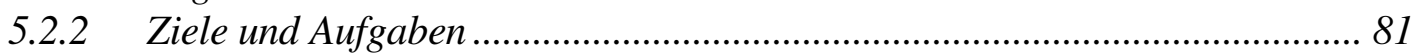

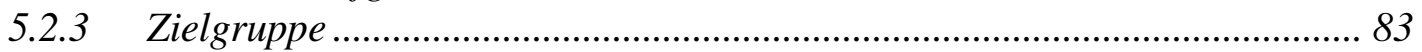

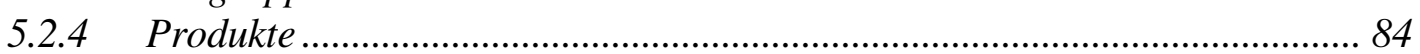

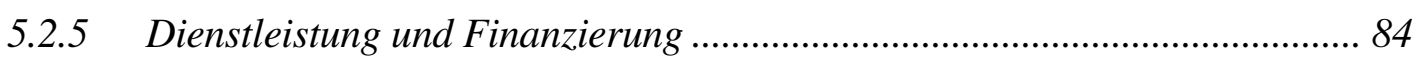

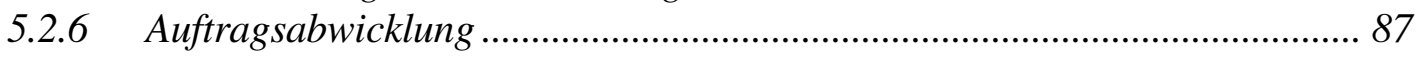

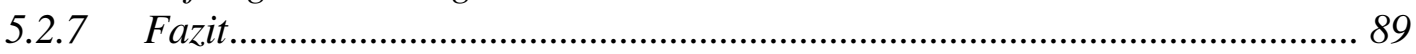

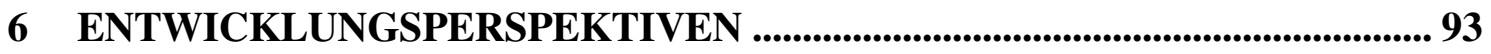




\section{ABBILDUNGSVERZEICHNIS}

Abbildung 1: TecNet - Organisationseinheit der Innovationsagentur ........................... 26

Abbildung 2: $\quad$ TecNet-Projekte nach Kundengruppen ............................................... 40

Abbildung 3: Abgeschlossene Projekte nach Technologiefeldern 1998-2001 ............. 45

Abbildung 4: Projektabwicklung des TecNet im Flussdiagramm ................................ 47

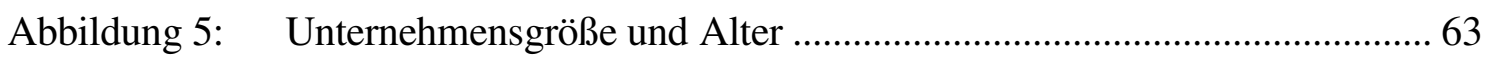

Abbildung 6: Leistungsangebot der Initiative „Feasibility Studies“ und des TecNet.. 76

Abbildung 7: „RechercheDienst“ - Organisationseinheit des FIZ Karlsruhe ............... 81

Abbildung 8: Projektabwicklung des FIZ im Flussdiagramm ...................................... 88

\section{TABELLENVERZEICHNIS}

Tabelle 1: $\quad$ Kundenansprache und Vermittlung externer Anfragen .......................... 36

Tabelle 2: $\quad$ Erträge externer Kundengruppen nach Rechtsform................................... 39

Tabelle 3: $\quad$ Anzahl interner/externer Projekte 1998-2001 …....................................... 41

Tabelle 4: $\quad$ Interne/externe Projekteinnahmen 1998-2001 ….................................... 42

Tabelle 5: $\quad$ Arbeitsstunden der Bereichsleitung 1999-2001 ....................................... 44

Tabelle 6: Anteil technologieorientierter Gründungen an allen Gründungen in \%. 58

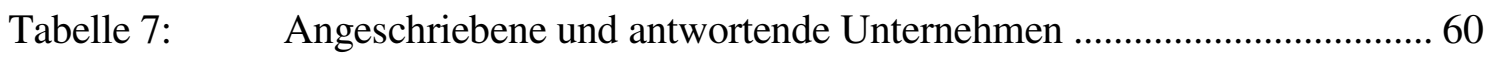

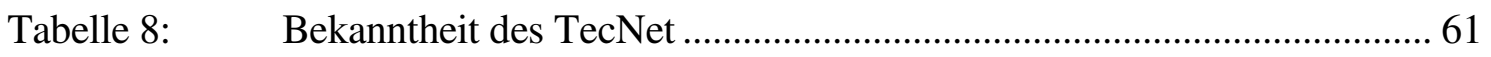

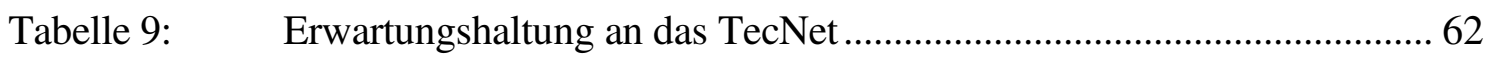

Tabelle 10: $\quad$ Nutzung der Marktinformationen des TecNet .........................................62 62

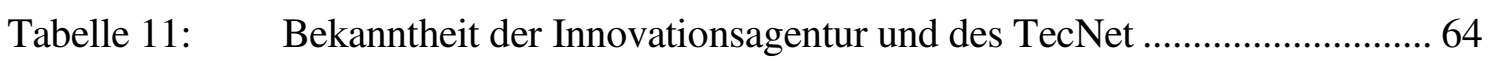

Tabelle 12: Recherche von Marktinformationen .......................................................... 65

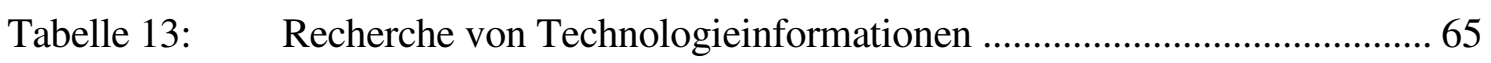

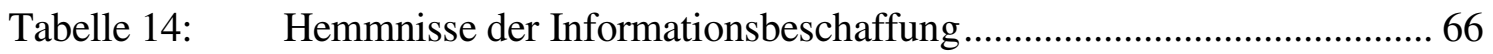

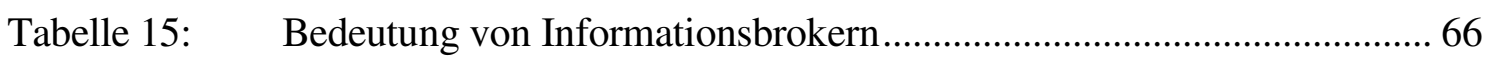

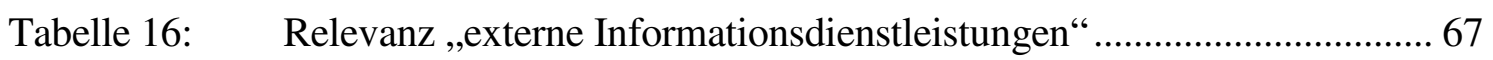

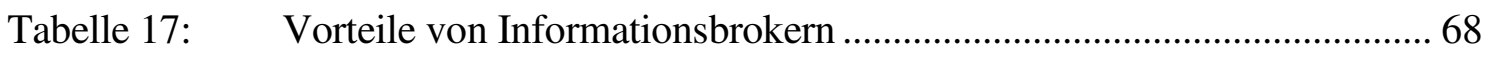

Tabelle 18: $\quad$ TecNet und FIZ Informationsdienste im Vergleich .................................. 79

Tabelle 19: $\quad$ Auftrag und Ziele des TecNet und des RechercheDienstes..................... 83

Tabelle 20: Kosten der Informationsrecherche im Vergleich ................................... 86 


\section{Vorbemerkung}

Die JOANNEUM RESEARCH Forschungsgesellschaft legt hiermit den Abschlußbericht für die Evaluation des „TecNet - Netzwerk für Markt- und Technologieinformationen der Innovationsagentur" vor. Dies stellt die erste Evaluation des betreffenden Tätigkeitsbereichs der Innovationsagentur dar. Die schriftlichen Unterlagen beurteilen die Arbeiten und Ziele von TecNet in den Jahren 1997 bis 2001. Der Ausgangspunkt für die Anfertigung dieser Unterlagen war eine Initiatives des „Rats für Forschung und Technologieentwicklung“. Auf Basis dieser Initiative wurde die Evaluation von TecNet durch das Bundesministerium für Wirtschaft und Arbeit (BMWA) ausgeschrieben. Die JOANNEUM RESEARCH Forschungsgesellschaft $\mathrm{mbH}$ hat ein Untersuchungsdesign für den Aufbau der Evaluation entwickelt und diese von Mai bis Juli 2002 durchgeführt. Das BMWA hat die Ergebnisse dieser Studie nicht beeinflusst; der Auftragnehmer trägt allein die Verantwortung.

Die Evaluation widmet sich der Zielerreichung, der Effektivität, der Effizienz sowie einem programmatischen und institutionellen Vergleich des „TecNet - Das Netzwerk für Markt- und Technologieinformationen“ (TecNet) der Innovationsagentur Ges.m.b.H. mit Sitz in Wien. Ziel ist es, die Aktivitäten des Netzwerks sowohl institutionsintern (Projektportfolio, Einnahmen, Personalstruktur etc.) als auch institutionsextern, das heißt aus Sicht der bisherigen sowie des potenziellen Klientels zu begutachten. Zur Beurteilung des internen Kostendeckungsgrads des Netzwerkes werden betriebswirtschaftliche Kennzahlen analysiert. Der externe Erfolg von TecNet wird anhand einer Kundenpotenzialanalyse und durch Befragungen bemessen. Die grundsätzliche Konzeption der Initiative wird sowohl mit Aktionslinien des „FFF“ als auch mit dem deutschen „Fachinformationszentrums Karlsruhe (FIZ)“ verglichen.

Die hier vorgelegten schriftlichen Unterlagen gliedern sich in sechs Kapitel und einen Anhang (Materialienband):

- Kapitel 1 fasst die Evaluationsergebnisse zusammen und beurteilt den TecNet-Erfolg

- Kapitel 2 skizziert die ökonomischen und politischen Hintergründe der Maßnahme

- Kapitel 3 analysiert Aufgaben, Arbeitsweisen und Zielerreichung von TecNet

- Kapitel 4 präsentiert die Ergebnisse der externen Befragung

- Kapitel 5 vergleicht die Konzeption von TecNet mit anderen Institutionen

- Kapitel 6 bietet Entwicklungsperspektiven als Diskussionsbeitrag

- Der Materialienband beinhaltet Einzeldaten und Vertragswerke dieser Evaluation.

Den Kapitel 2 bis 5 ist eine kurze Zusammenfassung der wichtigsten Ergebnisse beigefügt. Kapitel 6 dient als Diskussionsbeitrag zur zukünftigen Ausgestaltung von TecNet. Hinweise auf die verwendete Literatur sowie ergänzende Tabellen, Abbildungen und Materialien die der Evaluation zugrunde liegen finden sich im Anhang (Materialienband). 


\section{Zusammenfassung und Beurteilung}

\section{Allgemeine BemerKungen}

Ganz generell ist festzuhalten, dass das Evaluationsteam einen positiven Gesamteindruck von den Aktivitäten des TecNet gewonnen hat. Die durchweg hohe Qualität der bereitgestellten Informationen, die positive Resonanz der Kunden sowie das außerordentliche Engagement der Bereichsleitung sind besonders hervorzuheben. Die Evaluationsgespräche wurden in einer bereitwilligen und offenen Atmosphäre geführt. Auf Fragen, die sich aus dem Studium der schriftlichen Evaluationsunterlagen ergaben, antwortete die Bereichsleitung überzeugend. Das Evaluationsteam wurde bei seiner Arbeit von der Innovationsagentur und von TecNet nach Kräften und professionell unterstützt.

\section{EFFIZIENZ VON TECNET}

Zur Beurteilung der Effizienz von TecNet wurde in dieser Evaluation die vertragliche Zielsetzung herausgearbeitet, die Ausrichtung beschrieben, der Start-Up- und Entwicklungsprozess nachvollzogen und die Zielerreichung geprüft.

\section{ZIELSETZUNG UND AUSRICHTUNG}

Im Jahr 1997 wurde das TecNet als Organisationseinheit der Innovationsagentur mbH mit dem Ziel gegründet, die Finanzierungschancen von Technologieprojekten in österreichischen Unternehmen zu verbessern. Diese Zielsetzung wurde bis heute beibehalten. Das TecNet fungiert als Intermediär, um Financiers und Unternehmen über eine Technologieund Marktbewertung sowie durch eine Vermittlung von Experten zu besseren Entscheidungsgrundlagen zu verhelfen. Der aktuelle Vertrag (2001) präzisiert das Ziel, das TecNet als Anlaufstelle zu etablieren, um den Bedarf an rasch und preiswert verfügbaren, internationalen Marktinformationen zur Unterstützung von Finanzierungsentscheidungen bei High-Tech-Unternehmen oder Expertengutachten zur Einschätzung des Marktpotenzials von neuen Produkten und Technologien nachzukommen. $\mathrm{Zu}$ den Dienstleistungen des TecNet zählen (a) Recherchen zu Markt- und Technologieinformationen, (b) die Vermittlung von Expertengesprächen sowie (c) die Vermittlung von Gutachten.

Im Zeitverlauf ist die Entwicklung des TecNet durch zwei Phasen geprägt: eine Startphase (1997-1999) und eine Neuausrichtungsphase (1999-2001). In der Startphase war das Management des TecNet bemüht, institutionellen Kapitalgebern Technologieexperten zu vermitteln, die mit ihrer Expertise Finanzierungsentscheidungen verbessern helfen sollten. Gleichzeitig wurde mit diesem Vermittlungsangebot die Hoffnung verbunden, Finanzierungsrestriktionen bei Unternehmen zu beheben. Kerngedanke war es, Kapitalgebern ein qualifiziertes Expertennetzwerk und Informationen zur Verfügung zu stellen, damit aussichtsreiche Investitionsprojekte nicht mangels Technologiekompetenz abgelehnt würden. Dieses Konzept scheiterte in erster Linie mangels Nachfrage, weil die adressierten Kapitalgeber (Kreditinstitute, Venture Capital- 
Gesellschaften, Fördermittelgeber etc.) z.B. über eigene Netzwerke zu Experten verfügten, so dass an der Dienstleistung des TecNet vorbeimanövriert wurde. In der Neuausrichtungsphase verlagerte sich das Aufgabenprofil auf die Recherche von Marktund Technolgieinformationen. Der Anstoß zu diesem Angebot kam vor allem aus den anderen Organisationsbereichen der Innovationsagentur, dem TecMa und dem SeedCapital.

Im Zuge der Neuausrichtung wurde das Vermittlungsangebot im TecNet zwar beibehalten, trat aber in den Hintergrund der Aktivitäten. In diesem Zusammenhang trifft die offizielle Namensgebung „TecNet - Netzwerk für Markt- und Technologieinformationen der Innovationsagentur" heute nicht mehr den Kern der Tätigkeit. Seit dem Jahr 2000 stehen Informationsrecherchen im Zentrum der Dienstleistungen des TecNet. Hier wiederum sind es konkret Recherchen zu „technologiespezifischen Marktinformationen“ - und weniger die Recherche nach Technologie- und Marktinformationen bzw. die Vermittlung von Experten.

Grundsätzlich sei angemerkt, dass die Messlatte der Lerneffekte und Effizienz, die man an vergleichbare Organisationen nach vier Jahren ihrer Tätigkeit richtet, sich aufgrund der Neustrukturierung des TecNet auf die Jahre 2000 und 2001 reduziert.

\section{ZIELERREICHUNG}

Die Erreichung der schriftlich fixierten Ziele lässt sich wie folgt bewerten:

\section{Innovationspolitischer Beitrag}

Insgesamt leistet das TecNet zum zentralen Ziel der „Verbesserung und Verstärkung der Finanzierung von Technologieprojekten und als Hilfsmittel zur Umsetzung von Innovationen“ einen wertvollen Beitrag. Sein Dienstleistungsangebot dient Unternehmen und Kapitalgebern dazu, bessere Finanzierungsentscheidungen zu treffen. Präziser formuliert tragen die Informationen des TecNet dazu bei, dass die "Qualität von Investitionsentscheidungen“ verbessert wird. Dies hat zur Folge, dass Investitionschancen und -risiken besser abgeschätzt werden können, so dass es bei Unternehmen und Kapitalgebern weniger häufig zu Fehleinschätzungen und infolgedessen zu Fehlinvestitionen kommt. Auch wenn die Elastizität der Verbesserung in dieser Evaluation nicht quantifiziert werden kann, so sprechen sowohl der Kundenkreis als auch die Ergebnisse der Unternehmensbefragung für diese Einschätzung. Zu den unmittelbaren Kunden des TecNet zählen z.B. Fördermittelgeber wie die BÜRGES-Förderungsbank oder die Mittelstandsfinanzierungs-AG. Andere Kapitalgeber, wie Kreditinstitute und VCGesellschaften, profitieren mittelbar von den TecNet-Dienstleistungen, weil ihnen die Ergebnisse indirekt, z.B. über Business-Pläne zugehen. $81 \%$ der TecNet-Kunden geben an, die Informationen zur Abschätzung des Marktpotenzials bei Investitionsentscheidungen zu verwerten und loben die hohe Qualität der Informationen.

Das TecNet ermöglicht die frühzeitige Analyse der Marktchancen neuer Technologien. Es hilft Investoren das Ertragspotenzial zu ermessen und die Produktentwicklung an 
aussichtreiche Absatzmärkte anzupassen. Aus wirtschaftspolitischer Sicht ist das TecNet damit ein kleiner, aber wichtiger Bestandteil im Innovationssystem Österreichs.

\section{Zielgruppe}

Im Zeitverlauf ist festzustellen, dass TecNet sein Angebot stärker auf Jungunternehmen und Kapitalgeber ausrichtet und der Anteil der etablierten Firmen (älter als drei Jahre) kontinuierlich abnimmt. Dieser Trend der Jahre 2000 und 2001 erfüllt die vertragliche Zielvereinbarung, sich jungen High-Tech-Unternehmen sowie Geldgebern zuzuwenden. Trotz dieser Entwicklung sind High-Tech-Unternehmensgründer als unmittelbare Kunden jedoch eher selten. Vielfach werden diese über andere Organisationseinheiten der Innovationsagentur bedient. Die Organisationseinheiten SeedCapital und TecMa spielen dabei als Kunden des TecNet eine fundamentale Rolle. Insbesondere der hohe Anteil der von TecNet beratenen Erfinder (TecMa) sei hier genannt.

Im Zeitverlauf wurde der Adressatenkreis des TecNet in der Definition der Zielgruppe durch die Innovationsagentur bzw. das BMWA immer weiter ausgedehnt. Ursprünglich zielte das Angebot auf Banken und Venturegesellschaften ab. Heute umfasst die Zielgruppe: junge Technologieunternehmen, Geld- und Kapitalgeber (Banken, Business Angels, Beteiligungsgesellschaften etc.), Erfinder, Universitätsinstitute, KMUs, Industriebetriebe und die anderen Bereiche der Innovationsagentur. Obwohl das TecNet im Rahmen dieser Zielsetzung bemüht ist alle Einrichtungen zu erreichen, kann dies in keinem ausgewogenen Verhältnis erfolgen.

\section{Steigerung der Nettoeinnahmen}

Die Einnahmen des TecNet (ohne Studienzukauf) sind von rund 18.400 EURO im Jahr 1999 auf 22.000 EURO im Jahr 2001 angestiegen. Dennoch kann von einem Trend zur Eigenfinanzierung nicht gesprochen werden. Insbesondere der Einnahmenzuwachs zwischen den Jahren 2000 und 2001 fällt mit einer Steigerung von rund 600 EURO kaum ins Gewicht. Angesichts einer allgemeinen Verschlechterung des wirtschaftlichen Klimas in Europa, von denen die Finanzmärkte und in Folge auch Technologieinvestitionen betroffen waren, konnte das Niveau - das ist positiv zu werten - aus dem Jahr 2000 gehalten werden.

Bedenklicher stimmen dagegen die Finanzierungsquellen: Die externen Projekteinnahmen sind von rund 17.000 EURO (1999/2000) im Jahr 2001 auf knapp 10.000 EURO gesunken. Hier wurde das Vertragsziel, die Nettoeinnahmen durch externe Aufträge zu erhöhen verfehlt. Gegenfinanziert wurde dieser Einnahmenausfall mit internen Projekten. Hier erhöhte sich der Betrag von rund 3.800 EURO (2000) auf ca. 12.100 EURO (2001). Die Entwicklung ,weg vom Markt“, hin zu den ,internen Projekten“ lassen sich zwar mit Aspekten der Vertragsunsicherheit (BMWA) und mit der verschlechterten wirtschaftspolitischen Lage erklären, sollten aber im Hinblick auf eine höhere Eigenfinanzierungsquote die Ausnahme sein. 


\section{Steigerung der Projektanzahl}

In unmittelbarem Zusammenhang mit den Projekteinnahmen steht auch die Zahl der vom TecNet durchgeführten Projekte. Im Zuge der Neuausrichtung des TecNet (1999) konnte die Anzahl der durchgeführten Projekte zwar von 28 (1998) auf 41 Projekte (1999) gesteigert werden. Diese Wachstumsrate ließ in den folgenden zwei Jahren dann aber nach. Im Jahr 2000 und 2001 wurden jeweils 50 Projekte bearbeitet. Eine weitere Steigerung der durchgeführten Projekte wird dabei weniger durch den Markt, als vielmehr durch knappe Personalressourcen limitiert.

Angesichts schwächer notierter Kapitalmärkte und größerer Zurückhaltung bei Investoren, ist zu beobachten, dass es dem TecNet in 2001 schwerer fällt, kostenlose Projektanfragen in lukrative Aufträge überzuführen. Wurden 1999 von ca. 60 Anfragen noch 41 Projekte realisiert (68\%), stehen im Jahr 2001 insgesamt 99 Anfragen 50 Projekten (51\%) gegenüber. Nach einem erfreulichen Anstieg der externen Projekte mit Leistungsverrechung zwischen 1998 (3 Projekte) und dem Jahr 2000 (29 Projekte), wurde das für 2001 definierte Ziel die Zahl der durchgeführten Projekte (insbesondere für externe Auftraggeber) zu erhöhen mit nur 17 realisierten Projekten verfehlt. Dieses Verhältnis bleibt im Saldo negativ, auch wenn man die externen Projekte ohne Leistungsverrechung (Marketingmaßnahmen etc.) hinzuzählt.

\section{Erreichen von Zielvereinbarungen}

Positiv ist hervorzuheben, dass die internen Zielvereinbarungen der Bereichsleitung von TecNet mit der Geschäftsführung der Innovationsagentur punktgenau erreicht wurden. Ziel war es, im Zweijahreszeitraum 2000/2001 insgesamt 100 Projekte sowie ca. 80 Anfragen zu bearbeiten. TecNet hat dieses Ziel, trotz des schwächer notierten Technologiemarktes, erreicht. Verwunderlich ist allerdings, dass genau 50 Projekte im Jahr 2000 und 50 Projekte im Jahr 2001 bearbeitet wurden. Da nicht anzunehmen ist, dass TecNet weitere Projekte nach der Zielerreichung abgelehnt hätte, muss davon ausgegangen werden, dass große Anstrengungen unternommen wurden, genau 50 Projekte zu bearbeiten. Mit dieser Anzahl interner und externer Projekte werden, bei gegebenem Personalstand, allerdings auch Kapazitätsgrenzen erreicht. Insbesondere der Aufwand für externe Projekte ist hoch und könnte unter hoher Arbeitsbelastung dazu führen, das zur Zielerreichung interne Projekte bevorzugt werden.

\section{Anforderungen an das Marketing}

Bei den Zielsetzungen im Bereich Marketing wurden die Anforderungen weitgehend erfüllt. Bis heute hat sich das TecNet mit selbsterstellten Marketingunterlagen, einer eigenen Homepage, ,Success-Stories“ und zahlreichen Vorträgen auf Fachveranstaltungen seinen Kunden präsentiert. In einigen Fällen wurden auch kostenlose Recherchen durchgeführt, um strategisch wichtige Kundenkreise mit den Dienstleistungen des TecNet vertraut zu machen. Die Erstellung von Informationsmaterial z.B. die Drucklegung eines professionellen Image-Folders steht kurz vor der Fertigstellung.

Als sehr ansprechend präsentiert sich das TecNet im Außenbild. Das Corporate Design (Logo TecNet, Innovationsagentur, Farbgebung, Aufbau etc.) als Bestandteil der 
Marketingaktivitäten ist sehr gelungen, wobei einzig die lange Namensgebung „TecNet Das Netzwerk für Markt- und Technologieinformationen“ ggf. falsche Assoziationen hinsichtlich der Leistungen hervorruft. Die Unterlagen für Präsentationen sind vorbildlich aufbereitet und vermitteln die hohe Kompetenz im Bereich der Informationsvermittlung. Kritisch ist allenfalls anzumerken, dass hinsichtlich der Vortragsveranstaltungen und Pressearbeit einer allgemeinen Präsenz gegenüber einer gezielten Kundenansprache der Vorzug gegeben wird. Diese Omnipräsenz ist das unmittelbare Ergebnis der aus Sicht der Evaluatoren zu breit definierten Zielgruppe. Eine Konzeption der PR- und Medienarbeit, die sich in die Aktivitäten der Innovationsagentur einbettet ist schwer auszumachen. Dies ist aber nicht dem TecNet, sondern ganz allgemein bei der Innovationsagentur zu hinterfragen: Einerseits werden dem TecNet zahlreiche Marketingaktivitäten als eigenständige Zielsetzung auferlegt, andererseits wird von der Innovationsagentur ein eigener Organisationsbereich „Marketing“ aufgebaut. Hier mangelt es an einer konsistenten Gesamtstrategie.

\section{AnAlyse Der TeCNet-ProzesSe}

\section{Organisationsstruktur}

Das TecNet ist die kleinste eigenständige Organisationseinheit der Innovationsagentur. Diese Einheit wird lediglich von 1,5 Personen geführt, was angesichts der auferlegten Ziele und Aufgaben unzureichend ist. Das TecNet, das de facto aus nicht mehr als einer einzigen, als „Informationsbroker“ qualifizierten Person besteht, hat keine Vertretung, der/die bei Urlaub, im Krankheitsfall oder beim Ausscheiden der Bereichsleitung den Geschäftsbetrieb fortsetzen könnte. Die Bereichsleiterin konzentriert derzeit alle Erfahrungen, Kontakte und Geschäftsabläufe auf sich. Eine derartige Personalausstattung ist aus mehreren Gründen problematisch:

- Eine unflexible Personalpolitik verhindert Wachstumschancen und dämpft die Motivation der Mitarbeiter;

- Knappe Personalkapazitäten machen es praktisch unmöglich ein Österreichweit wahrgenommenes und nachgefragtes Dienstleistungsangebot zu offerieren. Einerseits ist die Kerntätigkeit der Informationsrecherche und Berichterstellung ortsgebunden, andererseits wird eine überregionale Vernetzung sowie ein Schulungs-/VortragsEngagement eingefordert;

- Mangelnde Arbeitsteilung überfordert auf längere Sicht selbst qualifizierte Personen;

- Mangelnde Delegationsmöglichkeiten führen zu einem Zwiespalt, sowohl interne als auch externe Kunden gleichberechtigt zu betreuen;

- Hohe „Overheads“ (Werbung, Akquisition und Verwaltungsaufgaben) beeinträchtigen das Kerngeschäft (Recherche und Dokumentation) und wirken sich negativ auf die Kostendeckung aus.

Verglichen mit anderen Organisationseinheiten der Innovationsagentur ist das TecNet personell nicht gewachsen und erweckt immer noch den Eindruck eines „Modells“. In Anbetracht der Zielsetzungen, der Zielgruppen, der internen Verwaltung- und externen Marketingaufgaben, des Wunsches nach Kostendeckung und aus pragmatischen Gründen (Vertretungsregelung etc.) ist das TecNet personell unterdimensioniert. 


\section{Vernetzung}

Immer unter dem Aspekt einer personellen Stärkung, ist aus Sicht der Evaluatoren eine Fokussierung auf die Zielgruppe der Unternehmensgründer und Kapitalgeber wünschenswert. Das heißt eine Ansprache im Rahmen von Informationsveranstaltungen sowie eine Vermittlung durch vernetzte Ansprechpartner, z.B. bei Förderinstitutionen (Technologieund Gründerzentren, dem FFF etc.). Hier könnte die arbeitsteilige Kooperation zwischen den einzelnen Vermittlungs- und Informationsstellen des Bundes und der Länder noch besser gelingen. Eine gegenseitige Wahrnehmung und intensivierte Zusammenarbeit der öffentlichen Intermediäre mit der privaten Finanzwirtschaft würde ohne Zweifel zu einer höheren Effizienz des staatlichen Unterstützungsangebots beitragen.

Eine solche Vernetzung von Vermittlungseinrichtungen zielt vor allem auf die Nutzung von Synergien ab, die sich beispielsweise aus der gemeinsamen Durchführung von Fachveranstaltungen, bei der komplementären Ansprache von Erfindern, Gründern, KMU, Industriebetrieben und Geldgebern ergeben. Die Vernetzung des TecNet ist zwar ,inhouse“ (Innovationsagentur) und mit den sie finanzierenden Einrichtungen (WiFi, BÜRGES Förderungsbank) gegeben, „extern“ erscheint diese Vernetzung jedoch noch ausbaubar. Im Vordergrund steht häufig die allgemeine Präsentation von Recherchetechniken auf einzelnen Fachveranstaltungen. Bis zum Erreichen einer wirklichen arbeitsteiligen Struktur, die auch über die Region Wien hinausreicht, sind allerdings größere Anstrengungen erforderlich, die in der aktuellen Konstruktion von TecNet kaum möglich sind.

Als Fernziel sollte sich ein System etablieren, in dem sich die Wissenschaft (Technische Universitäten, Fachhochschulen) mit ihrem Potenzial an Unternehmensgründern mit Förderern, Banken und dem TecNet als Bestandteil der Innovationsagentur regelmäßig austauschen, d.h. Interessenten und Kunden verzahnte Angebote und abgestimmte Lösungsmöglichkeiten vermitteln.

\section{Wettbewerbsumfeld}

Das TecNet bietet seine Leistungen in dem dynamischen Marktsegment der Informationsund Kommunikationsdienstleistungen an und entspricht im Profil den sog. „Informationsbrokern“. In Österreich, so zeigt eine Studie von KPMG (2001) - und das bestätigen auch die Recherchen des Evaluationsteams - gibt es keine unmittelbar mit dem TecNet vergleichbare Einrichtung. Das TecNet ist eine effiziente Marktlösung, wenn es Kapitalgeber, Erfinder, Gründer und jungen Technologieunternehmen durch bessere Informationen sowohl vor Fehlinvestitionen schützt als auch beiträgt Innovationsbarrieren $\mathrm{zu}$ überwinden. Ein solcher Beitrag gelingt durch das interne Auftragsverhältnis mit „TecMa“ (Technologie- und Patentverwertung der Innovationsagentur) und mit der BÜRGES-Förderungsbank. Für diese Kunden führt das TecNet zahlreiche Marktanalysen zum Potenzial, zur Verwertung und zur Vermarktung von Technologien und Erfindungen durch. Die success-stories der TecNet-Kunden belegen, dass sowohl Fehlinvestitionen vermieden als auch Kapital für Innovationen zur Verfügung gestellt wurde. Hier füllt das TecNet eine Marktnische aus und verbessert die Entscheidungsprozesse von Kapitalgebern, Unternehmern und kreativer Persönlichkeiten (Erfinder). 
Die Nachfrage nach den Dienstleistungen des TecNet ist kurz- bis mittelfristig als gut zu prognostizieren, weil sowohl Erfinder, Unternehmensgründer als auch junge High-TechUnternehmen das TecNet als „unabhängige Prüfstelle“ für die Marktgängigkeit ihrer Ideen bzw. neuer Produkte in Anspruch nehmen können. Unsicherheiten werden reduziert und die Markttransparenz wird erhöht. Im Rahmen von Finanzierungsfragen, BusinessPlänen oder Förderanträgen gelingt es, mit objektiven Informationen Kapitalgeber zu überzeugen. Auf der anderen Seite werden die Dienstleistungen des TecNet mit diesem Zweck auch von Kapitalgebern nachgefragt, um Marktkonzepte auf ihre objektive Realisierbarkeit hin zu prüfen. Insgesamt können sowohl die Kapital suchenden Firmen, als auch die Kapitalgeber, Finanzierungsentscheidungen durch solide Marktinformationen absichern lassen. Das es sich beim TecNet um eine „halbstaatliche“ Einrichtung (Innovationsagentur) handelt ist dabei nur von Vorteil. Die Mandanten des TecNet müssen nicht befürchten, dass Informationen an Dritte weitergeleitet werden, weil TecNet nicht profitorientiert ist.

\section{Auftragsabwicklung}

Die Ablauforganisation wurde durch eine professionellere Auftragsabwicklung in den vergangenen zwei Jahren deutlich verbessert. Inzwischen gibt es eine exakte Projektdokumentation, einheitliche Projektangebote, Endberichte und ein nachvollziehbares Abrechnungssystem. Dennoch sind die Prozesse insofern weiter verbesserungswürdig, als dass bei der Auftragsanbahnung Informationen geliefert werden, die der potenzielle Kunde unentgeltlich für eigene Recherchen nutzt. Hier besteht die Gefahr, dass das TecNet, wie bereits in der Startphase ,umlaufen“ wird. Die Tatsache, dass im Jahr 2001 rund die Hälfte aller Projektanfragen zu keinem Auftrag führten, sowie der lange organisatorische Vorlauf (Vorgespräch, Vorrecherche, Projektangebot) legen ein solches Verhalten, trotz des fairen Preisangebots nahe. Diese Form der Auftragsabwicklung ist im Hinblick auf einen ausgeglichenen Kostendeckungsgrad des Bereichs kaum effizient. Der Aspekt der Kostendeckung des TecNet ist allerdings, auch das muss erwähnt werden, kein explizites Ziel des Auftraggebers.

\section{Projektfinanzierung}

In der Projektabwicklung zeichnet sich ein Schlüsselproblem ab: Die Projektleitung des TecNet ist in der Akquisitionsphase arbeitstechnisch stark gefordert und bemüht dem Kunden in kürzester Zeit bestmögliche Ergebnisse zu präsentieren. Gleichzeitig wird ein Großteil der eigentlichen Arbeit im Vorfeld eines Vertrags erbracht - ohne dass diese Anbahnungskosten im späteren Vertrag gedeckt sind. Bei einer bereitwilligen Präsentation der Ergebnisse der Vorrecherche läuft das TecNet sogar Gefahr, den Kunden durch eine unfreiwillige Informationspreisgabe zu verlieren. Dieses Vorgehen ist nicht effizient und sollte überdacht werden.

In der Auftragsanbahnung würde man sich daher eine ausgewogenere Kosten/Ertragslage wünschen. Das TecNet sollte nicht zu viel Zeit auf die Vorrecherche legen und bei seinem Preisangebot durchaus in Kauf nehmen, dass ein Projekt auch mal nur zu 85 Prozent den Erwartungen des Kunden entspricht. Denkbar sind auch standardisierte Angebote oder Rahmenverträge, z.B. mit BÜRGES, die der Basisfinanzierung dienen. 
Ebenso könnten Leistungen auch über die Plattform „Internet“ elektronisch, z.B. als Formular zur Angebotserstellung angeboten werden. Solche Maßnahmen könnten Zeit sparen, den „Vorlaufaufwand“ reduzieren und es dem TecNet ermöglichen früher zu Marktpreisen abzurechnen bzw. früher ein Auftragsinteresse als verloren abzuhaken. Dieser Anspruch mag nach (real) zwei Jahren noch verfrüht erscheinen, sollte aber mittelfristig nicht aus den Augen verloren werden.

\section{EFFEKTIVITÄT VON TECNET}

Gemessen an den Erfahrungen mit Technologie- und Informationsvermittlungseinrichtungen in Deutschland, ist TecNet in der österreichischen Transfer-Landschaft richtig positioniert. Im Lichte der allgemeinen Zielsetzung der österreichischen Technologie- und Innovationspolitik wendet es sich jungen Technologieunternehmen, Unternehmensgründern und Erfindern sowie deren Kapitalgebern zu. Diese Zielgruppen leiten sich aus dem wichtigen Beitrag ab, den junge technologieorientierte Unternehmen zur Modernisierung der Wirtschaftsstruktur und für einen qualifizierten Arbeitsmarkt leisten. TecNet vermittelt seine Kernkompetenz „Recherche von Markt- und Technologieinformationen“" als klares Signal an Vertragspartner und Kunden. Andererseits zeigt diese Evaluation aber auch, das Neukunden zunächst von den Dienstleistungen des TecNet überzeugt werden müssen. Die Einbindung des TecNet als Organisationseinheit der Innovationsagentur ist vorteilhaft, weil auf diese Weise eine Vernetzung mit ähnlich gelagerten Förderbereichen und der BÜRGES-Förderungsbank gewährleistet ist.

Zur Beurteilung der Effektivität des TecNet wurden folgende Charakteristika herangezogen:

\section{Akzeptanz und Bekanntheit von TecNet}

Die Marktbedingungen des TecNet waren in den Jahren 1999 und 2000 für das TecNet besonders günstig, weil das allgemein positive Gründungsklima an den Kapitalmärkten dazu führte, dass sowohl Unternehmensgründer als auch Kapitalgeber die Dienste des TecNet in Anspruch nahmen. Seit Ende 2000 hat die Gründungseuphorie, die nicht zuletzt von den Erwartungen der Investoren (Business Angels, Kreditinstitute und VCGesellschaften) beflügelt wurde, merklich nachgelassen. Die Potenzialanalyse dieser Studie geht jedoch davon aus, dass in Österreich auch in 2001 mindestens 1.300 technologieintensive Unternehmen gegründet werden. Diese Unternehmen bilden ein ausreichendes Marktpotenzial für die Aktivitäten des TecNet. Der Standort Wien kommt dem TecNet zusätzlich zugute, da hier die höchsten Gründungsraten im gesamten österreichischen Bundesgebiet zu verzeichnen sind. Angesichts dieser Prognosen und in Anbetracht eines mäßigen Wettbewerberumfeldes sind die zukünftigen Vermarktungschancen der TecNet-Dienstleistungen als gut einzuschätzen.

In der schriftlichen Befragung geben die Kunden des TecNet an, nahezu ausschließlich Marktinformationen angefragt und erhalten zu haben. Diese Marktinformationen wurden von $81 \%$ der Klienten zur Abschätzung ihres Marktpotenzials verwertet. Das Kosten/Nutzen-Verhältnis wird als „fair“ oder sogar ,ausgezeichnet“ bezeichnet. Auch bei der Qualität sind die Kunden des TecNet außerordentlich zufrieden: 63 \% der Kunden 
beurteilen die Informationsqualität als „gut“; vier Kunden (25\%) vergeben sogar die Bestnote. Die vom TecNet aufbereiteten Informationen gehen bei allen Kunden nahezu vollständig in Entscheidungsprozesse ein. Die hohe Nutzungsfrequenz zeigt, dass die Leistungen des TecNet nicht nur angefragt, sondern auch verwertet werden. Sie sind damit ausgesprochen effektiv.

Darüber hinaus wird dem TecNet ein wirkungsvolles Management attestiert, welches dazu beiträgt, dass sich die meisten Firmen eine regelmäßige Kooperation wünschen. Bis auf einen Unternehmensgründer, der seine Auftragsziele erreicht hat, würden alle bisherigen Kunden das TecNet wiederholt mit einem Rechercheauftrag betrauen.

Wenig effektiv ist das TecNet angesichts des Anspruchs, dass es allen Firmen und Kapitalgebern in Österreich offen stehen soll. Den meisten Unternehmen und Kapitalgebern ist das „Netzwerk TecNet“ unbekannt: von 66 zufällig in Österreich ausgewählten technologieintensiven Unternehmen, Kapitalgebern und Wissenschaftseinrichtungen geben $86 \%$ an, dass sie das TecNet nicht zu kennen. Anhand dieser Befragung zeigt sich, dass sich das TecNet noch nicht als „Marke“ bzw. als Leistungsträger der Innovationsagentur etablieren konnte. Insbesondere Unternehmensgründern und KMU ist das TecNet als Institution neu.

Grundsätzlich betrachten die meisten Unternehmen die Leistungen eines Informationsbrokers als Ergänzung der eigenen Recherchen (56\% TecNet-Kunden; $48 \%$ NichtTecNet-Kunden). Insbesondere die Nicht-TecNet-Kunden sehen in diesem Angebot keine Alternative $\mathrm{zu}$ eigenen Recherchen. Ein Informationsbroker, so die verbreitete Auffassung, kann zwar umfassender, nicht aber aktueller recherchieren als das Unternehmen selbst.

\section{Vergleich zu anderen Informationsdienstleistern}

Das TecNet wurde in seinem Leistungsangebot (a) programmatisch mit den Feasibility Studies des FFF in Österreich und (b) institutionell mit dem RechercheDienst des Fachinformationszentrums Karlsruhe in Deutschland verglichen

Ein programmatischer Vergleich der Angebote des TecNet und des FFF zeigen Unterschiede im Dienstleistungsschwerpunkt. Obwohl die technologiepolitischen Ausgangsüberlegungen die gleichen sind, werden in beiden Maßnahmen unterschiedliche Informationsinhalte erhoben. Während sich TecNet in den vergangenen zwei Jahren auf die Recherche von Marktinformationen konzentriert hat, ist die Förderung des FFF auf die Prüfung und Umsetzung einzelner Technologien ausgerichtet. Das TecNet deckt mit seinem Leistungsangebot somit die ,wirtschaftlichen Perspektiven“ eines Finanzierungsprojekts ab, während der FFF mit seinen Feasibility Studien die „technische Realisierung“ auslotet. Es handelt sich um ein komplementäres Förderinstrument.

Anzuregen wäre dennoch eine Kooperation des TecNet mit Forschungsinstitutionen, die Studien im Rahmen der FFF-Förderung für Unternehmen durchführen. Durch die Vernetzung erlangen spezialisierte Forschungseinrichtungen nicht nur den Einblick in das technologische Potenzial einer Erfindung, sondern werden auch auf die Bedeutung der Absatzmärkte hingewiesen. Hier sind auf Seiten der theorielastigen Forschungsinstitute

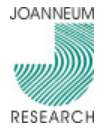


Lern- und Erfahrungseffekte zu erwarten, weil zukünftig bei der Beurteilung neuer Technologien auch die Marktpotenziale hinterfragt würden. Das Unternehmen selbst profitiert von einer Expertise mit hoher Informationsqualität. Es würden nicht nur technologische Aspekte berücksichtigt, sondern auch wichtige Informationen zu Märkten und Wettbewerbern erhoben. In einer solchen Expertise würden zum einen die FuEKosten der Erfindung abgeschätzt, und zum anderen die Verwertungs- und Marktchancen beurteilt. Die Kausalkette von der „Invention zur erfolgreichen Innovation“ würde somit um eine Entscheidungshilfe, die Unsicherheiten reduziert, bereichert. Die Kombination der Technologieinformationen der Programme „Feasibility Studies“ bzw. „Start UpFörderung“ mit den technologiespezifischen Marktinformationen des TecNet verbessern Rentabilitätsrechnungen und helfen bei der Kalkulation des ROI (Return-on-Investment).

Im Institutionenvergleich zeigt sich, dass das österreichische TecNet und der deutsche „RechercheDienst“ zwei eigenständige Organisationseinheiten staatlicher Informationsinfrastruktur sind. Beide Dienste recherchieren wissenschafts- und unternehmensrelevante Informationen in Datenbanken mit dem Ziel, Prozesse der Wissens- und Entscheidungsfindung zu verbessern.

Ausgangspunkt des deutschen Dienstleistungsangebots ist das Hauptprodukt des FIZ Karlsruhe: der Aufbau, die Pflege und die Erweiterung umfangreicher, internationaler Technologiedatenbanken. Auf diesen Produkten aufbauend, hat sich der RechercheDienst als Unterstützungsservice für informationssuchende Kunden etabliert. Qualifizierte Experten sind beim RechercheDienst für die Recherche in technologiespezifischen Fachdatenbanken verantwortlich. Aufgrund identischer Abfragestrategien und angesichts einer fachübergreifenden Zusammenarbeit, können sich die Rechercheure im Bedarfsfall gegenseitig vertreten und auch Fachvertreter aus anderen Abteilungen des FIZ hinzuziehen. Hier zeigt sich eine größere Effizienz als sie beim TecNet vorherrscht.

Der Kunde zahlt in Deutschland grundsätzlich für das Hauptprodukt: den Datenbankzugriff, die enthaltene Information und den Datenbankoutput. Er hat allerdings die Wahl, ob er Datenbankzugriffe selbständig (Online-Service) ausführt, oder durch die Experten des RechercheDienstes ausführen lässt. Die Dienstleistung des RechercheDienstes wird dann gesondert in Rechnung gestellt. Diese systematische Ablauforganisation wird von einem detaillierten Preissystem flankiert, das Interessenten und Kunden die Informationskosten transparent macht. Die aktuellen Preise lassen sich - im Unterschied zum TecNet - über das Internet abrufen.

Das TecNet vermarktet gegenüber dem RechercheDienst nicht seine Datenbanken sondern seine Recherchedienstleistung als Hauptprodukt. Die Informationsleistung wird marktspezifisch und kundenorientiert erbracht. Sie ist nicht auf Spezialdatenbanken beschränkt, sondern erfordert eine Sichtung mehrerer Informationsquellen. Ein effiziente Arbeitsteilung ist aufgrund der aktuellen Personalausstattung nicht möglich. Die Informationsrecherche ist vergleichsweise weniger formalisiert und systematisiert. Im Unterschied zum deutschen RechercheDienst werden Skaleneffekte dadurch kaum aufgebaut bzw. genutzt. Darüber hinaus wird nicht Schlüsselbegriff sondern im Ziel- 
kontext des Kunden recherchiert. Die Marktrecherche unterscheidet sich von der reinen Technologierecherche durch eine höhere Komplexität und einen höheren Aufwand.

Wie bereits an anderer Stelle ausgeführt, kommt hinzu, dass beim TecNet vor einer Auftragsvergabe Vorrecherchen durchgeführt werden. Da das TecNet diese aufwendigen Vorrecherchen nicht kalkuliert, Informationskosten (Datenbankzugriffe) nicht separat berechnet und vor der Auftragsvergabe Informationsquellen preisgibt, ist der Deckungsbeitrag gering. Zudem werden einzelne Arbeitsschritte nach einer Auftragsvergabe wiederholt, so dass es zu Redundanz kommt. Auch wenn man argumentiert, dass somit die Kosten der Vorrecherche im eigentlichen Projekt berücksichtigt werden, ist dieser Gedankengang nicht stichhaltig: Da der eigentliche Aufwand in der Suche der Informationen liegt, die nach erfolgreicher Vorrecherche in Teilbereichen abgeschlossen ist, werden in der Hauptrecherche nur noch die erfolgreichen Suchschritte wiederholt und berechnet. Die nicht erfolgreichen Rechercheschritte sind für das TecNet „sunk costs“, die zwar entstanden, nicht aber auf den Kunden abgewälzt werden. Hier besteht ein wesentlicher Unterschied zu anderen Informationsbrokern wie z.B. dem RechercheDienst.

\section{ABSCHLIESSENDE BEMERKUNGEN}

Das Evaluationsteam der JOANNEUM RESEARCH Forschungsgesellschaft mbH ist zu der Überzeugung gelangt, dass die Dienstleistungen des TecNet den an eine derartige Institution zu stellenden innovationspolitischen Anforderungen genügen und der Beitrag zur Verbesserung von Investitionsentscheidungen bei der Finanzierung von Technologieprojekten in vielen Fällen gegeben ist. Die Evaluatoren interpretieren den erhaltenen Auftrag aber auch so, dass die erhaltenen Eindrücke und daraus entwickelte Anregungen für die weiteren Arbeiten bzw. für die strategische Ausrichtung von TecNet Bestandteile des Evaluationsberichts sein sollen. Die Evaluationsgruppe ist davon überzeugt, dass durch eine Verbesserung der Organisation, die Fokussierung auf eine begrenzte Zielgruppe und durch eine größere Bekanntheit das vorhandene Potenzial des TecNet noch besser erschlossen werden kann.

In diesem Sinne sind die an verschiedenen Stellen des Berichts angebrachten kritischen Anmerkungen zu verstehen. Es sei nochmals darauf hingewiesen, dass einige Defizite dem TecNet nicht direkt anzulasten sind, sondern die Folge einer nicht immer konsequenten Strategie der Innovationsagentur, aber auch des Auftraggebers sind. Unklare Vertragssituationen (Finanzierungsentscheidungen) wirken sich immer nachteilig auf die Motivation der Betroffenen und auf das mühevoll erarbeitete Außenbild aus. Gerade unter diesem Gesichtspunkt wünschen die Evaluatoren dem TecNet eine bessere Planungsperspektive sowie eine erfolgreiche Fortführung seiner Rechercheleistungen. Sie sind davon überzeugt, dass sich die Dienste auch in Zukunft ihre hohe Relevanz bei Erfindern, Technologieunternehmen, Förderern und Kapitalgebern beibehalten. Die Bereichsleitung des TecNet bringt die dazu notwendigen Voraussetzungen mit und hat die erforderliche Kompetenz durch ihre Arbeiten nachgewiesen. 


\section{Wirtschaftspolitischer Handlungsbedarf}

\subsection{WISSENS- UND TECHNOLOGIETRANSFER}

Wissen ist heute - neben Arbeit und Kapital - ein wichtiger Produktionsfaktor. Wissen ist der Rohstoff für Innovationen und Ausgangspunkt für die ökonomische und technologische Leistungsfähigkeit moderner Volkswirtschaften. Die Wissensgenerierung erfolgt sowohl durch ein differenziertes Bildungssystem mit ausreichenden Qualifizierungsmöglichkeiten als auch durch die alltägliche Verarbeitung von Informationen, insbesondere von Markt- und Technologieinformationen. Seit Mitte der achtziger Jahre wird dieses Wissen über Märkte und Technologien mit Hilfe der Informations- und Kommunikationstechnologien gesammelt, kanalisiert und von denjenigen als Wettbewerbsvorteil eingesetzt, die über einen Zugang zu diesem Wissen verfügen.

Wirtschaftlicher Erfolg und gesellschaftliche Kohäsion hängen, so die Innovationsforschung, zu einem großen Teil von der Fähigkeit ab, sich spezifische Informationen anzueignen und zielgerichtet weiterzuverarbeiten. Die Effizienz einer Volkswirtschaft wird damit maßgeblich von der Fähigkeit der Menschen, Wissenschaftseinrichtungen und Unternehmen determiniert, die in der Lage sind, sich externes Wissen zu eigen zu machen. Dieses Problem, das in Fachkreisen unter dem Begriff der „Absorptionskapazität" diskutiert wird, ist jedoch nicht nur an eine technologische Kompetenz geknüpft. Insbesondere bei Unternehmen sind die vorherrschende Innovationskultur als auch die Prozesskompetenz wesentliche Faktoren, die die Absorptionskapazität ausmachen. Prozesskompetenz meint dabei die Fähigkeit, Erfolgspotenziale strategisch zu planen, Innovationsideen systematisch $\mathrm{zu}$ generieren und $\mathrm{zu}$ selektieren sowie die systematische Informationsbeschaffung über neue Technologien sicher $\mathrm{zu}$ stellen. Weiterhin sind Entscheidungen über die Art der Technologiebeschaffung zu treffen, die Innovationsprojekte zu steuern und innovationsorientierte Organisations- und Anreizstrukturen zu entwickeln.

Mit einer verantwortungsbewussten Legislative und der Bereitstellung einer leistungsfähigen Infrastruktur (Bildung, Wissenschaft, Verkehrswege, Kommunikation etc.) verfolgt der Staat das Ziel, optimale Bedingungen für wirtschaftliches Wachstum, gesellschaftliche Entwicklung und kulturelle Vielfalt zu schaffen. Sein vitales Interesse ist es, ein Fundament für sozialverträgliches Wirtschaftswachstum zu legen, das Arbeitplätze schafft und soziale Gerechtigkeit sichert - grundsätzlich ohne die Absicht in die freie Entfaltung und Selbstregulierungskräfte des Marktes einzugreifen. Im Rahmen dieser Ziele ist die Bereitstellung von und der Zugang zu Informationen, die das Wissen über Märkte und Technologien ausmachen, von steigender Bedeutung. Angesichts zunehmend kürzerer Innovations- und Produktzyklen entscheidet sich mit dem Zugang zu Informationen die Wettbewerbsfähigkeit einer Volkswirtschaft. Es ist daher nicht erstaunlich, dass die führenden Industrienationen dem „Wissens- und Technologie- 
transfer" eine hohe Aufmerksamkeit schenken. Im Verantwortungsbereich der Wirtschafts- und Innovationspolitik haben die meisten OECD-Länder es sich zur Aufgabe gemacht, der nationalen Wirtschaft und Wissenschaft im internationalen Informationswettlauf gute Startbedingungen $\mathrm{zu}$ verschaffen. Insbesondere junge, sowie kleine und mittlere Unternehmen sollen durch bessere Informationskanäle in die Lage versetzt werden, Wissen über Märkte und Technologien zu absorbieren die ihnen - häufig in Nischen - Marktvorteile sichern und Innovationen ermöglichen.

\subsection{AUSGANGSLAGE UND HANDLUNGSBEDARF IN ÖSTERREICH}

Ziel der österreichischen Technologie- und Innovationspolitik ist es, die technologischen und wirtschaftlichen Rahmenbedingungen für Unternehmen durch Wissens- und Technologietransfermaßnahmen zu verbessern. Die Förderung dieses Transfers ist dabei von besonderer Bedeutung, weil der beschleunigte Zugang zu Know-how es Industrieunternehmen und Dienstleistern ermöglicht auf neue Entwicklungen schneller zu reagieren und diese in eigene Produkte oder Herstellungsprozesse einfließen zu lassen. Die beschleunigte Verfügbarkeit von Informationen ist für die österreichische Wirtschaft essentiell, da sie dazu beiträgt innovative Produkte bzw. Prozesse in kürzeren Zeitphasen zu entwickeln, zu implementieren und zu vermarkten.

$\mathrm{Zu}$ diesem Zweck haben sich in Österreich mehrere Technologietransfereinrichtungen etabliert, die in erster Linie eine Vermittlerfunktion zwischen Wissensangebot und Wissensnachfrage wahrnehmen sollen. Eine der bedeutendsten Initiativen, Technologietransfer in Österreich zu fördern, war in den letzten Jahren das Schwerpunktprogramm des ITF, das zur Zeit in einer von der TIG und dem ERP abgewickelten Programm des BMWA, „Protec“, seine Fortsetzung findet. Des weiteren haben sich in Österreich mehrere Technologietransfereinrichtungen etabliert, die die beschriebene Vermittlerfunktion zwischen Wissensangebot und Wissensnachfrage wahrnehmen sollen. Erwähnenswert sind hier Transfereinrichtungen im universitären Bereich (Außeninstitute der Universitäten), die außeruniversitäre Forschungsszene, Christian Doppler Labors, eine etablierte Szene von Technologie-, Innovations- und Gründerzentren, des weiteren Einrichtungen wie Profactor Steyr, aber auch die Kompetenzzentrenprogramme Kplus, Kint und Knet.

Es besteht damit ein Netzwerk aus Institutionen (,Intermediäre in Wissen und Technologie“), die in öffentlichen Forschungseinrichtungen, oder bei wirtschaftsnahen Einrichtungen beheimatet sind. Darüber hinaus wurden eigenständige Wissens- und Technologietransferinstitutionen in Form von Transferagenturen und Transfernetzwerken eingerichtet. Ein wichtiges Ziel dieser Intermediäre ist es, den Austausch von Wissen und Technologie zwischen wissenschaftlichen Einrichtungen und Unternehmen zu fördern, die Forschungsergebnisse aus der Wissenschaft einer kommerziellen Nutzung zugänglich zu machen und die Innovationsaktivitäten von Unternehmen durch die Verbesserung des Zugangs zu Fremdkapital zu erhöhen. 


\subsection{MASSNAHMEN DES BMWA UND DIE ENTSTEHUNG DES „TECNET“}

Der wichtigste Indikator für die steigende Bedeutung der Erschließung und Nutzung von Wissen in Form externen Know-hows ist das Instrument des institutionalisierten Wissensund Informationstransfers. Die „Innovationsagentur“ zählt zu den beschriebenen Institutionen und befindet sich zu 50\% im Besitz des Bundesministeriums für Wirtschaft und Arbeit (BMWA), der Wirtschaftskammer Österreich (20\%), der BÜRGES Förderungsbank Gesellschaft m.b.H. (20\%) sowie anderer Sozialpartner (9\%). Als Institution des Bundes, der die Mehrheitsanteile hält, hat sie den Auftrag mit ihren Geschäftsbereichen Unternehmen bei der Finanzierung von Technologien sowie bei der Verwirklichung von Innovationen und Ideen zu unterstïtzen.

Gegen Ende der 90er Jahre identifizierte die Innovationsagentur aus den Geschäftsbereichen, die mit Kreditinstituten und Förderbanken kooperieren den Bedarf, ,das technische und wirtschaftliche Bewertungspotenzial von Universitäten, Beratern, etc. und hinsichtlich Qualität, Umfang, Zeit und Kosten“ zu eruieren (Materialien 2, S. 6). Etwa zur gleichen Zeit bestand der politische Wille, die Technologie- und Wirtschaftsaktivitäten in Österreich zu forcieren. Aus Privatisierungserlösen wurde die sogenannte erste Technologiemilliarde gespeist. Durch die Erfahrungen der Innovationsagentur und ausländischer Transferzentren motiviert, entschied sich das BMWA 1997 - auch nachdem die „Technologiebewertung“ im Expertenentwurf zum technologiepolitischen Konzept der Bundesregierung als Aktionsfeld definiert worden war - auf Anregung der Innovationsagentur für den Aufbau einer Patentverwertungsagentur und eines Netzwerkes für Technologiebewertung. ${ }^{1}$ Die Innovationsagentur bot für dieses Netzwerk eine geeignete Plattform, da hier bereits Experten mit Unternehmen, Wissenschaft und Wirtschaftsförderern kooperierten.

Der wirtschaftpolitische Handlungsbedarf für den Aufbau eines Netzwerkes für Technologiebewertung wurde aus einem mangelnden Technologieverständnis auf Seiten von Kapitalgebern (Kreditinstitute, Venture-Capital Gesellschaften) einerseits, und immer komplexeren Innovationen bzw. Technologien auf Seiten der kapitalsuchenden Unternehmen (Start-Up Firmen, KMU) andererseits, abgeleitet. Mit dem geplanten Netzwerk zur „Technologie- und Marktbewertung“ sollte nicht nur die Finanzierungsentscheidung der Financiers verbessert und erleichtert werden, sondern auch Technologienehmern ein Hilfsmittel zur Umsetzung ihrer Innovationen an die Hand gegeben werden (Materialien 25, Expertengespräch [3]).

Den Ideenanstoß zu TecNet kam aus einer Studie der Steinbeiss-Stiftung heraus, die einen solchen Bedarf ortete und aus der Beobachtung, dass die Banken sich aus der Technologiebewertung sukzessive zurückzogen. Die Ansiedelung bei der Innovationsagentur hatte unter anderem den (praktischen) Grund, dass kurz zuvor die „Verschwesterung“ der BÜRGES-Förderbank mit der Innovationsagentur stattgefunden hatte. Andere Banken werteten dies als ein Signal, dass die BÜRGES-Förderbank nun Know-how im technologischen Bereich aufweisen könne (Materialien 25, Expertengespräch [4]). 


\section{TecNet - Netzwerk der Innovationsagentur}

Das TecNet als Organisationseinheit der Innovationsagentur mbH ist Gegenstand dieses Kapitels. Chronologisch werden die Verträge, die Ziele und Aufgaben sowie die Entwicklung des TecNet analysiert (Materialien 1-4). Die Fakten dieses Abschnitts resultieren aus einem umfangreichen schriftlichen Fragenkatalog, den das TecNet im Rahmen dieser Evaluation beantwortet hat (Materialien 5). Aufbauend auf diesem Fragebogen wurden längere Gespräche mit der Bereichsleitung des TecNet, wie auch mit der Geschäftsführung der Innovationsagentur geführt (Materialien 25). Darüber hinaus wurden die zuständigen Mitarbeiter des BMWA zu den Hintergründen und zur technologiepolitischen Zielsetzung befragt. Die Leistungen des TecNet werden an den schriftlich fixierten Zielen gemessen und in einem abschließenden Fazit diskutiert.

\subsection{VERTRÄGE}

Das BMWA hat mit der Innovationsagentur Ges.m.b.H. als Auftragnehmer über den Evaluierungsgegenstand zwei Verträge abgeschlossen:

- „Aufbau einer Patentverwertungsagentur und eines Netzwerkes für Technologiebewertung“ (GZ: 98.322/1-IX/B/10/97), basierend auf den Inhalten des Angebots der Innovationsagentur Ges.m.b.H vom 10. Juli 1997. Das Vertragsverhältnis begann rückwirkend zum 1. November 1997 und endete mit dem 31. Juli 2000.

- „TecNet - Das Netzwerk für Markt- und Technologieinformationen“ (GZ 98.100/12-IV/B/17/01), basierend auf den Inhalten des Angebots der Innovationsagentur GmbH vom 30. Mai 2001. Das Vertragsverhältnis begann rückwirkend zum 1. Januar 2001 und endet - vorbehaltlich einer vorzeitigen Auflösung des Vertragsverhältnisses als Folge negativer Evaluierungsergebnisse - mit 31. Dezember 2003.

Die Angebote der Innovationsagentur Ges.m.b.H bilden einen integrierenden Bestandteil der Verträge mit dem BMWA (Materialien 1 - 4).

\subsection{ERRICHTUNG UND ORGANISATIONSSTRUKTUR}

Die Errichtung einer Institution zur Technologie- und Marktbewertung wurde 1997 von der Innovationsagentur angeregt und in engem Zusammenhang mit Aktivitäten der Patentverwertung gesehen. Aufgrund einer hohen Affinität zu modernen Technologien sollten beide Institutionen unter dem Dach der Innovationsagentur als kooperierende Einheiten integriert werden. Im Umfeld der übrigen, bei der Innovationsagentur beheimateten Förderbereiche plante das Management „ein Netzwerk von Experten aufzubauen, das zur Beurteilung der mit der Einführung, Anwendung und Vermarktung 
von neuen Technologien verknüpften Risiken“ dient. Von Beginn an war vorgesehen, das heutige „TecNet“ als eigenständige Organisationseinheit, gleichberechtigt neben den existierenden vier bzw. im Aufbau befindlichen Geschäftsbereichen der Innovationsagentur, einzugliedern. Die Geschäftsbereiche werden mittels eigener Auftragsverträge als Programme von verschiedenen Ministerien finanziert. Das TecNet positioniert sich 2002 wie im folgenden Organigramm abgebildet:

Abbildung 1: TecNet-Organisationseinheit der Innovationsagentur

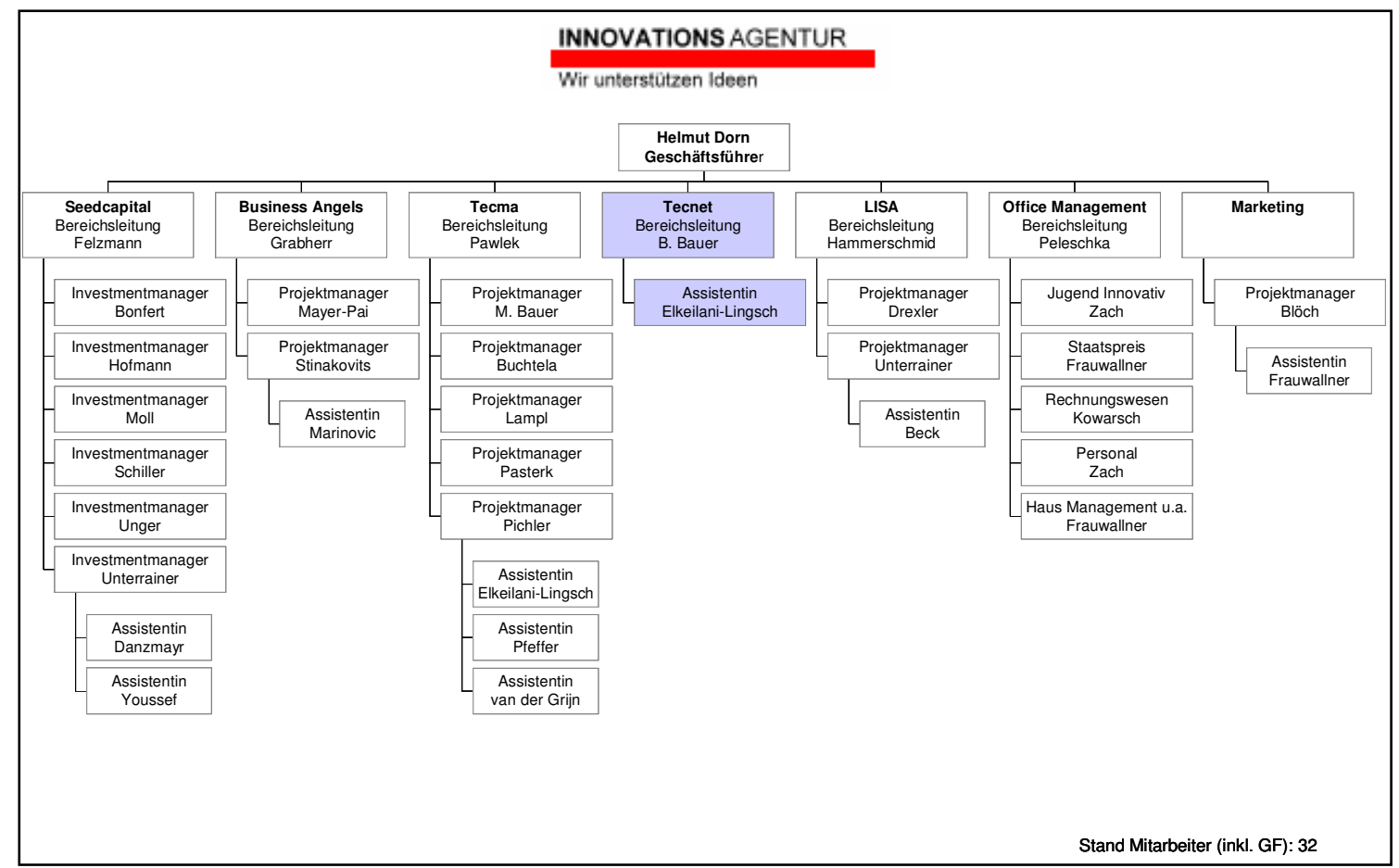

Quelle: Innovationsagentur (2002)

Das TecNet besteht nicht als eigene Rechtsform, sondern ist eine Organisationseinheit der Innovationsagentur Ges.m.b.H. Die Leitung der Innovationsagentur hat Herr Dr. Dorn inne; die Leitung von TecNet wurde 1997 Frau Dr. Hölbing übertragen und 1999 von Frau DI Bauer übernommen. Da es sich bei der Errichtung von TecNet um ein bis dato in Europa einmaliges Projekt handelte, zu dem keine institutionellen Erfahrungen vorlagen, wurde der Organisationsbereich kompetent, aber bewusst „klein“ angelegt. Insgesamt sind im TecNet 1,5 Personen beschäftigt. Hierbei handelt es sich seit 1997 um eine projekterfahrene Akademikerin sowie um eine zuarbeitende Sekretärin, wobei letztere der Organisationseinheit zu 50 Prozent ihrer Vollzeitbeschäftigung zur Verfügung steht. Die „Gleitzeit mit Kernarbeitszeit“" ist die vorherrschende Arbeitszeitregelung. Der zentrale Arbeitsort mit der für TecNet notwendigen Infrastruktur befindet sich am Sitz der Innovationsagentur in Wien.

Das TecNet hat zwischen seiner Errichtung 1997/1998 und 2001 keine personelle Aufstockung erfahren. Im Vergleich mit den übrigen Organisationseinheiten der Innovationsagentur ist es der personell kleinste Bereich. Dies verwundert insofern, als dass im Unterschied $\mathrm{zu}$ den übrigen Bereichen die Projektakquisition mit 
Kostenerstattung zu seinen zusätzlichen Aufgaben zählt. Obwohl die Zielsetzungen damit um einiges anspruchsvoller sind, spiegelt sich dieser Anspruch nicht in der Personalstruktur wieder (vgl. Abbildung 1).

\subsection{ZIELE UND AUFGABEN}

\subsubsection{Zielsetzung}

Der Vertrag des BMWA mit der Innovationsagentur vom 10. Juli 1997 formuliert die „Verbesserung und Verstärkung der Finanzierung von Technologieprojekten“ als wichtigste Zielsetzung (Materialien 1, § 5.3.2. und § 5.4.2). Durch eine „Technologieund Marktbewertung“, die das Aufgabenspektrum von TecNet umschreibt, sollten „Finanzierungsentscheidungen von Financiers verbessert und erleichtert werden, andererseits Technologieunternehmen ein Hilfsmittel zur Umsetzung ihrer Innovationen“ an die Hand gegeben werden.

Der derzeit gültige Vertrag des BMWA mit der Innovationsagentur vom 30. Mai 2001 greift diese Ziele erneut auf und betont zusätzlich, dass kleine Unternehmen, die sich weder Infrastruktur für aussagekräftige Marktanalysen leisten können, noch das Knowhow haben, ,gleiche Chancen wie große Unternehmen“ erhalten sollen (Materialien 3, § 3.1, Abs. II, S. 2). In diesem Zusammenhang ist es das Ziel das TecNet als Anlaufstelle zu etablieren ,um den Bedarf an rasch und preiswert verfügbaren, internationalen Marktinformationen zur Unterstützung von Finanzierungsentscheidungen bei High-TechUnternehmen oder Expertengutachten zur Einschätzung des Marktpotenzials von neuen Produkten und Technologien nachzukommen [...]“ (Materialien 4, § 1, Abs. II). Darüber hinaus wurde in 2001 der Ziel- und Aufgabenkatalogs des TecNet explizit um Marketingaktivitäten erweitert, die TecNet, neben den genannten Zielen, zur Erstellung von Informationsmaterialien und Vorträgen verpflichtet. Diese Marketingaktivitäten sollten zur selbstgesetzten Zielerreichung in 2001 beitragen (vgl. Materialien 3, § 4):

- Erhöhung der Nettoeinnahmen (insbesondere durch externe Aufträge)

- Erhöhung der Zahl der durchgeführten Projekte (insbesondere für externe Auftraggeber)

- Gezielte Öffentlichkeitsarbeit und Aufbau von TecNet als Marke.

Die jährlichen Zielvereinbarungen der Bereichsleitung von TecNet mit der Geschäftsführung der Innovationsagentur umfassen (a) die Einnahmen, als Maß für Nachfrage und Kundenzufriedenheit, (b) die Zahl der durchgeführten Projekte sowie (c) eine Erhöhung des Bekanntheitsgrads des TecNet. Bei der Zahl der durchzuführenden Projekte wurden für den Zweijahreszeitraum 2000/2001 insgesamt 100 Projekte sowie ca. 80 Anfragen als Messlatte vereinbart. 


\subsubsection{Startphase 1997-1999}

In der Anfangsphase von TecNet (1997) wurden Anstrengungen unternommen, vor allem Experten, Gutachter bzw. Gutachten an Kapitalgeber (Kreditinstitute, Venture Capital Gesellschaften, Business-Angels, Förderbanken) zu vermitteln. Die Innovationsagentur plante in diesem Zusammenhang ,im Umfeld von $\mathrm{i}^{2}$, der Börse für Business Angels, dem Seedfinancing Programm und der Patentverwertungsagentur ein Netzwerk von Experten aufzubauen, das zur Beurteilung der mit der Einführung, Anwendung und Vermarktung von neuen Technologien verknüpften Risiken diente. Es ging dabei um eine Technologieund Marktbewertung (TEMA), die den Financiers von Technologieunternehmen und Technologieprojekten deren Finanzierungsentscheidungen erleichtern“ sollte (Materialien $2, \S 6.1 .2$, Abs. II).

Dabei war vorgesehen die Leistung nicht, wie ansonsten bei öffentlich finanzierten Gesellschaften üblich, kostenlos bereitzustellen: „Die Vermittlungstätigkeit zwischen Experten und Financiers wird von der Innovationsagentur gegen teilweisen Kostenersatz durchgeführt. Die Kosten (Honorare) für die Technologie- und Marktbewertung sind vom jeweiligen Auftraggeber zu bezahlen“ (Materialien 2, § 6.1.2, Abs. III).

Das geplante Dienstleistungsangebot baute auf einer Bedarfsanalyse bei Kreditinstituten sowie auf den bis dahin gesammelten Erfahrungen der Innovationsagentur in den Programmen $\mathrm{i}^{2}$ und SeedCapital auf. Die Zielgruppe waren vor allem Banken und Venturegesellschaften, zu denen die Innovationsagentur im Rahmen von SeedCapital und $\mathrm{i}^{2}$ und die BÜRGES Förderungsbank seit langem regelmäßige Kontakte pflegte. „Die bereits bewährte Zusammenarbeit mit diesen Zielgruppen in den Geschäftsbereichen der Innovationsagentur und BÜRGES Förderungsbank kann in Marketingaktionen für die Technologiebewertung eingebracht werden“ (Materialien 2, § 6.1.2, Abs. IV).

Der Bedarf wurde im Wesentlichen diagnostiziert aus (a) regelmäßigen Anfragen von Kreditinstituten an die BÜRGES Förderungsbank sowie (b) aus einer Reduzierung der Technologiekompetenz bei den üblichen Kapitalgebern (Banken). Kreditinstitute, so die Diagnose für 1997/98, investierten nicht weiter in ihre Organisationseinheiten bzw. technologiekompetente Mitarbeiter, da technologisches Wissen - insbesondere in den IuK-Technologien - zu schnell veraltet. Dieser Trend wurde nicht nur in Österreich, sondern auch im benachbarten Ausland beobachtet. Aufgrund dieser Marktlage, bot es sich an, das TecNet als „Expertennetzwerk“ bzw. „Gutachterpool“ zu etablieren, um die vorhandene bzw. entstehende Marktlücke im Segment Technologiebewertung mit einem eignen Angebot zu schließen. Es erschien rentabel, Kapitalgebern ein Expertennetzwerk als Alternative zu eigenen Ressourcen (Mitarbeiter, Weiterbildung etc.) anzubieten. Die Zielgruppe waren daher vorrangig Banken und VC-Gesellschaften, die für Finanzierungsentscheidungen auf Expertenurteile angewiesen sind, und diese als Humankapital „,inhouse“ nicht mehr vorhalten wollten. Gleichzeitig zielte das TecNet mit seinem Angebot darauf ab Finanzierungsrestriktionen bei Unternehmensgründungen zu beheben, weil mit einem Expertennetzwerk die Hoffnung verbunden wurde, Kapitalgeber von Finanzierungsrisiken bzw. -unsicherheiten zu befreien, so dass Kredite gewährt würden, die zuvor aufgrund mangelnder Informationen ablehnt worden waren. 
Das TecNet sah es als seine Aufgabe an, den Kreditinstituten, innovationsbereiten Unternehmen und Erfindern, einschlägige Experten an Universitäten, technischen Hochschulen, außeruniversitären Forschungseinrichtungen etc. im In- und Ausland zu vermitteln. In diesem Verständnis wollte das TecNet Netzwerkpartner, Vermittler und Moderator sein, um Kapitalgebern Finanzierungsentscheidungen $\mathrm{zu}$ erleichtern und Innovatoren kompetente Ansprechpartner anzubieten (Materialien 25, Expertengespräch [2] und [4]).

\subsubsection{Neuausrichtung 1999-2000}

Der gewünschte Erfolg des beschriebenen Konzepts der Startphase von TecNet blieb bis 1999 jedoch im Dienstleistungsangebot „Vermittlung von schriftlichen Gutachten über High-Tech-Produkte und -dienstleistungen“" aus. Aufgrund mangelnder externer Nachfrage musste man das ursprüngliche Arbeitskonzept zur definierten Zielerreichung als gescheitert einstufen: Die Dienstleistungen ,,wurden von Banken und anderen Geldgebern nicht nachgefragt“" (vgl. Materialien 3, § 3.3, Abs. I, Satz 2).

Die Gründe des Scheiterns bei externen Kunden lagen vor allem in (a) der unzureichenden Bereitschaft von Kreditinstituten bzw. Firmen für eine reine Vermittlungsleistung einen Preis zu zahlen sowie in (b) bereits etablierten Kontakten von Kapitalgebern mit der Wissenschaft, öffentlichen Technologiezentren und Experten. Existierende Netzwerke bei den ursprünglich als Kunden vermuteten Kreditinstituten und Finanzierungsgesellschaften führten dazu, das z.B. Gutachter von den Auftraggebern direkt angesprochen wurden, ohne die Leistungen von TecNet in Anspruch zu nehmen. Aufgrund dieser Direktansprache gelang es TecNet nicht, sich als ein „Makler für Sachverständige“" zu positionieren.

In dieser Erkenntnis kam es zu einer Zäsur, die sich gravierend auf die Aufgaben und Arbeitsinhalte von TecNet auswirkte. Die intern geleisteten Recherchen zu Markt- und Technologieinformationen für die Bereichen TecMa und SeedCapital führten dazu, dass sich ein neuer Tätigkeitsschwerpunkt entwickelte: die Durchführung von Rechercheprojekten. Die Nachfrage von Recherchediensten bzw. Informationen ergab sich aus der Problematik, dass start-up Firmen potenzielle Kapitalgeber mit Markt- und Technologieinformationen (Wettbewerbsumfeld, Marktpotenzial etc.) versorgen müssen, aufgrund ihrer dünnen Kapitaldecke aber keine spezialisierten Consultants beauftragen können. Da bei Business-Plänen, im Rahmen der Kreditantragstellung oder bei Förderanträgen jedoch verlangt wird, nicht nur das technologische, sondern auch das Vermarktungspotenzial abzuschätzen, entdeckte TecNet auf diesem Feld seine Marktnische: „Ein Bedarf an rasch und preiswert verfügbaren, internationalen Marktinformationen zur Unterstützung von Finanzierungsentscheidungen bei High-Tech-Unternehmen wurde dagegen deutlich“ (Materialien 3, § 3.3, Abs. II). Nach Analyse des Marktes und Durchführung der ersten Projekte wurden im Laufe des Jahres 1998 die bis heute von TecNet angebotenen Dienstleistungen definiert. Zu diesen zählen (Materialien 3, § 3.1, Abs. I):

- Recherche zu Markt- und Technologieinformationen (inkl. Aufbereitung der Informationen und Bericht) 
- Vermittlung von Gesprächen mit Experten und Analysten

- Vermittlung von Gutachten

Inzwischen lassen im Bereich des ,seed capital“ Förderbanken und Kapitalgeber über TecNet z.B. Markt- bzw. Technologieinformationen durch Rechercheaufträge auf ihren Wahrheitsgehalt prüfen, oder vermitteln Kunden im Vorfeld eines Kredit-/Förderantrags. Aber auch etablierten Unternehmen wird das Angebot unterbreitet, aufwendige Marktrecherchen professionell durchführen $\mathrm{zu}$ lassen: Informationen $\mathrm{zu}$ einer angestrebten Internationalisierung, neuen Produktstrategien oder die Marktmacht der Wettbewerber etc. sind bedeutende Entscheidungshilfen, die Unternehmen als Grundlage für ihre Planungen benötigen. Darüber hinaus sieht das TecNet für seine Klienten eine bessere Chance, um bei öffentlichen Förderungen z.B. bei EU-Anträgen letztlich erfolgreich zu sein.

Das TecNet formuliert seine Leistungen wie folgt: „Mit der Vermittlung von Markt- und Technologieinformationen werden Business-Pläne von High-Tech-Start-ups untermauert, strategische Entscheidungen hinsichtlich Produktentwicklung und Vermarktung von Technologie-Unternehmen unterstützt und Finanzierungsentscheidungen von Geldgebern erleichtert" (Materialien 4, § 3.1, Abs. II, S. 1). Darüber hinaus versteht sich das TecNet als Beratungseinheit innerhalb der Innovationsagentur. Hier unterstützt es die benachbarten Bereiche TecMa und SeedCapital bei der Umsetzung wissenschaftlicher Forschungsergebnisse in kommerzielle Produkte bzw. bei der Suche nach Lizenznehmern.

\subsection{ZIELGRUPPEN UND STRATEGISCHE POSITIONIERUNG}

\subsubsection{Zielgruppen}

In der Startphase des TecNet (1997-1999) zählten vor allem Geld- und Kapitalgeber zur Zielgruppe des TecNet. Im Einzelnen werden im Angebot vom 10. Juli 1997 (Materialien 1, § 6.1.2, Abs. I, S. 1; Abs. VI, S. 1) die folgenden zu adressierenden Kunden genannt:

- Banken

- Venturegesellschaften und

- andere Bereiche der Innovationsagentur (hier: Patentverwertung)

Nach der Neuausrichtung des TecNet (1999-2001) wurde die Zielgruppe ausgeweitet auf (Materialien 3):

- Junge High-Tech-Unternehmen,

- Geld- und Kapitalgeber (Banken, Business-Angels, Beteiligungsgesellschaften,...)

- Erfinder, Universitätsinstitute, KMUs, Industriebetriebe und

- andere Bereiche der Innovationsagentur 
Im Selbstverständnis des TecNet umfasst diese Zielgruppe explizit High-Tech-Gründer und neben jungen Firmen auch kleine Technologieunternehmen sowie Consultants.

\subsubsection{Strategische Positionierung}

Das Klienten-/Kundensegment von TecNet setzt sich zusammen aus (a) internen Projektaufträgen (andere Organisationseinheiten der Innovationsagentur) sowie (b) aus externen Anfragen bzw. Aufträgen.

Während in der Startphase von TecNet die erhofften Aufträge von externen Geld- und Kapitalgebern ausblieben, wurde in der Folgezeit das Kundensegment auf High-TechStart-up-Unternehmen sowie auf Erfinder, Universitätsinstitute, KMUs, und Industriebetriebe ausgedehnt. Im Übergang von der Startphase zur Neuausrichtung änderte sich auch das Dienstleistungsspektrum: Während ursprünglich die Vermittlungstätigkeit zwischen Experten und Financiers im Vordergrund stand, wurde diese Leistung um die Recherche zu Markt- und Technologieinformationen erweitert. Es ist zu betonen, dass der Klientenkreis sowie das Dienstleistungsangebot erweitert und nicht, wie man angesichts der Entwicklung in der Startphase hätte vermuten können, ersetzt wurde. Der Schwerpunkt der Leistungen hat sich eindeutig auf die „Ermittlung von Marktinformationen für High-Tech-Bereiche“ verlagert, die nunmehr gelegentlich durch Technologieinformationen ergänzt werden (vgl. Materialien 3, § 3.1, Abs. III, S. 2).

\subsubsection{Abgrenzung}

Das TecNet grenzt sich seit 1999 stärker gegenüber anderen Beratungseinrichtungen wie dem Techinform, den WiFis, den Außeninstituten der Universitäten sowie gegenüber freien Beratern ab. Schwerpunkt sind, ,internationale Marktinformationen für High-TechProjekte“. Das TecNet sieht seine Aufgabenschwerpunkte auch in 2000 und 2001 darin, sich von vergleichbaren Anbietern abzugrenzen. So werden weder reine Patentrecherchen, noch reine Technologierecherchen durchgeführt. Ebenso wenig will das TecNet Umfragen durchführen, juristische Recherchen anbieten oder aufwendige Marktanalysen betreiben.

\subsubsection{Netzwerkcharakter}

Das TecNet versteht sich als Partner und Bestandteil eines verzweigten Netzwerks von Unternehmen, Wirtschaftsförderern und Geldgebern.

- Kooperationsverträge wurden mit Landesorganisationen und AplusB-Zentren (Wirtschaftsstandort Vorarlberg, EKS Salzburg, Tec Tirol, CATT Linz etc.) abgeschlossen.

- Durch die Gesellschafterstruktur (20\% der Innovationsagentur sind im Besitz der Wirtschaftskammer, weitere $20 \%$ im Besitz der BÜRGES Förderungsbank) besteht ein direkter Kontakt zu Fördermittelgebern.

- Über informelle Kooperationen mit dem WiFi bestehen Kooperationsverträge z.B. mit dem Stanford Research Institute und dem Massachusetts Institute of Technology sowie mit der Gartner Group, die auch Consultingleistungen beinhalten. 
- Die Innovationsagentur bzw. das TecNet ist Mitglied des TIN-Netzwerkes des FFF.

- Enge, netzwerkähnliche Kontakte bestehen auch zu Datenbankanbietern, Technologie-Experten und Fachverbänden von Industrie und Wirtschaft.

\subsection{PRODUKTE UND MARKTSEGMENT}

\subsubsection{Art der Dienstleistungen}

Grobe Schätzungen gehen davon aus, das sich das weltweite Wissen innerhalb von sechs bis sieben Jahren verdoppelt. Täglich werden über 3.000 Bücher publiziert und mehr als 200.000 wissenschaftliche Journale verbreiten weltweit Informationen. Diese werden in rund 8.500 Datenbanken, Zeitungsarchiven, Wissenschafts- und Dokumentationszentren oder in Patentämtern gespeichert. Hinzu kommt eine unüberschaubar hohe Zahl an wirtschaftswissenschaftlichen Printmedien, die in Form von Berichten, Magazinen, Fachzeitschriften, Working-Papers etc. publiziert werden. Die Recherche in Datenbanken, die Informationssuche im Internet und das Bündeln von Informationen ist für Unternehmen, Wissenschaft, Forschung und Behörden inzwischen eine große Herausforderung. Das TecNet sieht seine Leistung darin, Informationen so verfügbar zu machen und derart zusammenzustellen, dass sie von Unternehmen und Kapitalgebern sinnvoll genutzt werden können. Unternehmen, die sich Informationen nur mit großem Zeitaufwand beschaffen könnten, sparen durch die Tätigkeit des TecNet wichtige Ressourcen (Zeit, Geld, Personal etc.). Darüber hinaus werden sie in die Lage versetzt sich durch den gewonnenen Informationsvorsprung Wettbewerbsvorteile zu verschaffen.

Das „Produkt“ des TecNet besteht aus Leistungen, die in der Recherche, Vermittlung und Beratung liegen und in einem schriftlichen Zwischen- bzw. Endbericht zusammengefasst werden (Materialien 16). Im einzelnen bietet das TecNet folgende Serviceleistungen (Materialien 19) an:

- Recherche zu allenfalls vorhandenen Markt- und/oder Technologieinformationen bzw. Studien. Durch Zugang zu internationalen Markt- und Technologiedatenbanken wird ermittelt ob und welche Studien bereits verfügbar sind. Diese Studien werden als ganzes oder in Auszügen für den Auftraggeber beschafft.

- Nach Absprache und falls erforderlich, Kontaktaufnahme mit TecNet-Netzwerkpartnern (Nationale und internationale Berater, Experten aus universitären und außeruniversitären Forschungsbereichen, Experten aus der Industrie). Recherche im Internet, Anfragen bei Fachverbänden, Außenhandelsstellen etc.

- Vermittlung von Gesprächen mit Experten und Analysten zu Markt- und/oder Technologiefragen

- Vermittlung von Gutachten

- Suche nach potenziellen Lizenznehmern

- Vorträge/Workshops (Recherchieren/Informieren für innovative Produkte) 
Ein Endbericht bildet das abschließende Produkt von TecNet an einen Auftraggeber. Der Abschlußbericht ist in einer einheitlichen, übersichtlichen und ansprechenden Form gestaltet. Er gliedert den Auftrag in (a) eine Beschreibung der Aufgabenstellung, (b) eine Zusammenfassung der Ergebnisse sowie (c) Einzelergebnisse. Darüber hinaus werden (d) Referenzen und ein (e) Anhang mit Zusatzinformationen ausgewiesen.

\subsubsection{Marktsegment}

Das TecNet ist gemäß der NACE-Klassifizierung den Klassen

- 72 Datenverarbeitung und Datenbanken (72.60.1 Informationsvermittlung) und

- 74 Erbringung von unternehmensbezogene Dienstleistungen (74.13.1 Marktforschung)

zuzurechnen. Die NACE Rev.1 dient dazu, die wirtschaftlichen Tätigkeiten von Unternehmen, Betrieben und anderen statistischen Einheiten in allen amtlichen Statistiken einheitlich zu erfassen. Sie baut auf der durch EG-Verordnungen verbindlich eingeführten statistischen Systematik der Wirtschaftszweige in der Europäischen Gemeinschaft auf. Statistik Austria verzeichnet im Bereich „72 Datenverarbeitung und Datenbanken“ bis 1998 rund 10.320 umsatzsteuerpflichtige Unternehmen, wobei die Zahl der Firmen von 1995 (3.830 Firmen) an deutlich gestiegen ist. Obwohl keine offiziellen Zahlen bis 2001 vorliegen ist davon auszugehen, das sich diese Zahl weiter gesteigert hat, so dass TecNet mit seinen Dienstleistungen in einem dynamischen Wachstumsmarkt anzusiedeln ist. Im spezifischen Marktsegment „Informationsvermittlung“ sind 1997 lediglich 11, 1998 insgesamt 19 und 1999 insgesamt 20 Unternehmen in der ISIS-Datenbank von Statistik Austria registriert. Aufgrund des Gründungsbooms im Jahr 2000/2001 bei IKTUnternehmen ist die Anzahl heute auf 30 bis 40 Unternehmen zu schätzen. Die Wirtschaftsprüfungs- und Beratungsgesellschaft KPMG stellt in einem Gutachten zu wirtschaftspolitischen Aktivitäten in Europa dennoch fest, dass mit den Leistungen des TecNet vergleichbare Angebote privater Informationsbroker in Österreich nicht existieren (vgl. Materialien 22, S. 12).

\subsubsection{Wettbewerbsumfeld}

Auf internationaler Ebene sind vor allem Unternehmen wie REUTERS Ltd. zu nennen, die auf Informationen über Finanz- und Onlinemärkte spezialisiert sind. In Österreich sind Unternehmen, die sich ausschließlich auf die Recherche von Markt- und Technologieinformationen spezialisiert haben nur schwer zu identifizieren, weil die meisten kommerziellen Anbieter entweder ein breiteres oder ein spezialisierteres Spektrum an Dienstleistungen aufweisen. So werden Marktinformationen beispielsweise von CREDITREFORM AUSTRIA oder DUN \& BRADSTREET AUSTRIA in Form von Wirtschaftsdaten angeboten, um Unternehmen $\mathrm{zu}$ helfen ihr Kreditrisiko bei Handelsgeschäften zu reduzieren. Gegenüber diesen Firmen verfügt TecNet über ein umfassenderes Informations- und Berichtssystem. Andererseits gibt es auch zahlreiche EDV-/Informationsdienstleister, wie z.B. die „S@F EDV Dienstleistungen Mag. Josef Aschauer“, die „Weigl \& Weigl Informationsvermittlung“ oder das „WWW Searchcenter Prinzersdorf“ die sehr branchenspezialisiert ihre Leistungen anbieten. Die Firma S@F hat 
sich z.B. auf die Bereiche Wirtschaft und Reisen konzentriert. Weigl \& Weigl beschäftigt sich mit der Vermittlung von Ausschreibungsunterlagen, und das WWW Searchcenter Prinzerdorf bietet Unternehmen ein „Infobroking“ in Form von Informationen über Mitbewerber und Patentauskünften an. Bei all diesen Unternehmen gibt es einmal engere, einmal weitere Überschneidungen mit dem Leistungsangebot des TecNet.

Insgesamt muss man den jungen Markt der Informationsbroker in Österreich als heterogen beschreiben: es sind derzeit keine kommerziellen Firmen auszumachen, die sich mit dem gleichen Profil wie das TecNet am Markt präsentieren. Darüber hinaus gibt es keine Interessensverbände, Vereine oder Lobbys, die speziell das Berufsbild des Informationsbrokers oder hierauf spezialisierte Firmen vertreten. In vielen Fällen gibt es Überschneidungen im Dienstleistungsangebot mit etablierten Unternehmen sowie mit Wissenschaft- und Fördergesellschaften. Die Kernkompetenzen von Unternehmensberatungsgesellschaften, Wirtschaftsberatern, Handelsauskunfteien oder anderen Einrichtungen liegen im Allgemeinen auf spezifischen Produkten, bei denen Markt- und Technologierecherchen als eine Komponente bzw. als Vorarbeiten für das eigentliche Angebot (Produkt) zu werten sind. Beispielsweise kann ein Berater seine unternehmensindividuelle Leistung nur dann erbringen, wenn er über Erfahrungen und detaillierte Kenntnisse über den Markt, die Prozesse und die Technologie seinen Kunden verfügt. Somit führt er in seinem Beratungssegment regelmäßig Recherchen und Marktbeobachtungen durch, die als ein Auftrag unter vielen auch der Leistung von TecNet entsprechen könnte.

$\mathrm{Zu}$ den unmittelbaren Wettbewerbern bzw. zu den Anbietern mit gleichwertigen Dienstleistungen wie das TecNet zählen allenfalls

- kommerzielle Unternehmensberatungsgesellschaften,

- kommerzielle „Informationsbroker“ sowie

- öffentliche Förder- und Wissenstransferagenturen

Aufgrund des beschriebene Leistungsspektrums von TecNet ist dieses mit dem noch jungen Unternehmenszweig der „Informationsbroker“ zu vergleichen. Informationsbroker verstehen sich als externe Informationsvermittler die im Kundenauftrag in externen Datenbanksystemen bzw. Berichtssystemen Auftragsrecherchen übernehmen. Zu diesen Recherchen zählen beispielsweise:

- Firmenprofil-Recherchen

- Handelsregister Recherchen

- Markenrecherchen

- Marktrecherchen

- Monitoring von Datenbanken

- Patentrecherchen

- Presserecherche

- Technische Fachinformationsrecherchen (Maschinenbau- und Verfahrenstechnik) 
Als Referenz zur Einordnung des Wettbewerbsumfeldes von TecNet ist festzustellen, dass in Deutschland zur Zeit ca. 6.000 bis 7.000 InformationsbrokerInnen als Angestellte in privaten und öffentlichen Unternehmen arbeiten. Zusätzlich schätzt die Deutsche Gesellschaft für Informationswissenschaft und Informationspraxis e.V. (DGI), dass etwa 1.000 Personen als Freiberufler tätig sind und dass der Bedarf an qualifizierten Bewerbern in den kommenden Jahren weiter zunehmen wird.

\subsubsection{Wettbewerbsvorteile}

Die nachfragerelevanten Vorteile des TecNet liegen in seinem Recherche-Know-how, sowie in der schnellen und diskreten Auftragsabwicklung. Es verfügt über den Zugang zu spezialisierten Marktdatenbanken, die sich aufgrund langfristiger Verträge und Kosten insbesondere für junge bzw. kleine Unternehmen nicht lohnen. Der Zugang zu international renommierten Marktforschungsinstituten sichert die Seriosität der Informationen. Darüber hinaus lassen sich Studien auch kapitel- und seitenweise über das TecNet beziehen, so dass Informationskosten nur bedarfsgerecht für den Kunden anfallen. TecNet recherchiert nicht allgemein für einen breiten Markt, sondern auftragsbezogen und damit individuell. Ein wesentlicher Vorteil des TecNet gegenüber kommerziellen Konkurrenten ist die öffentliche Förderung. Obwohl das TecNet die Philosophie verfolgt, das die erbrachte Dienstleistung auch wertgeschätzt und damit einen Preis erhalten muss, wird die Infrastruktur (Geschäftsräume, IKT-Ausstattung, Personal etc.) vom Staat finanziert. Kooperationen mit Programmbausteinen des WiFi Österreich ermöglichen es zudem - ergänzend zu den Marktrecherchen von TecNet - auch Patent- und Technologierecherchen zu vermitteln bzw. durchzuführen.

\subsection{MARKETING UND AUFTRAGSPLANUNG}

\subsubsection{Zielsetzung}

Die Werbemaßnahmen des TecNet wurden im Vertrag vom 30. Mai 2001 explizit als Eigenleistung von TecNet geregelt. Die Etablierung von TecNet als Anlaufstelle für Unternehmen etc. soll durch gezielte Marketingaktivitäten erfolgen. $\mathrm{Zu}$ diesen zählen (Materialien 4, §1, Abs. II, S. 2):

- das Erstellen von Informationsmaterial (Folder, Texte mit neuen success stories)

- das Erstellen eines Konzepts für eine gezielte PR- und Medienarbeit

- die gezielte Pressearbeit (Pressetext und Artikel für relevante Printmedien)

- Vorträge im Rahmen von Gründer-Veranstaltungen (vier Vorträge im Rahmen des „BOB - Best-of-Biotech Businessplanwettbewerbs“)

- Vorträge bei Kredit und Kapitalgebern sowie Multiplikatoren wie dem WiFi

TecNet beschreibt dieses Angebot als Bestandteile seiner langfristigen Werbestrategie, wobei Empfehlungen bzw. Referenzen von Kunden zu den wichtigsten Marketing- 
instrumenten zählen. Das Netzwerk zielt darauf ab, mit guter Arbeit und Endberichten zu überzeugen. Die „Werbemessage“ an Kunden und potenzielle Kunden lautet:

- TecNet bietet den Zugang zu internationalen, professionellen Marktanalysen und Marktinformationen.

- Das Angebot von TecNet ist zugeschnitten auf

- High-Tech-Unternehmensgründer oder

- High-Tech-Produktentwickler oder

- ihre Geldgeber

- TecNet ist Spezialist - liefert Qualität, ist fachlich kompetent, seriös, diskret, preiswert und kostenpflichtig.

\subsubsection{Umsetzung}

Die Umsetzung dieser Werbemitteilung erfolgt nach Angaben von TecNet durch Projektarbeit, Kooperationen, Vorträge/Workshop-Beiträge, Internet (Homepage, Mailing), Folder und Pressearbeit. Bei der differenzierten Betrachtung der Instrumente die TecNet zur Kundenansprache nutzt, zeigt sich, dass der persönliche Kontakt (,face to face“) bzw. die Ansprache auf Fachveranstaltungen etwa $80 \%$ der Werbeaktivitäten ausmacht. Rund $20 \%$ der Werbung entfällt auf Informationsmappen und diverses Werbematerialien der Innovationsagentur. Eine Verbreitung der Bekanntheit durch öffentliche Träger oder Ministerien im Rahmen eigener Publikationen ist nicht festzustellen. Um das Verhältnis interner und externer Projektanfragen im Rahmen des Marketing zu bemessen, wurden die in Tabelle 1 gelisteten Vermittlungspartner identifiziert.

Tabelle 1: $\quad$ Kundenansprache und Vermittlung externer Anfragen

\begin{tabular}{lcccc}
\hline & $\mathbf{1 9 9 8}$ & $\mathbf{1 9 9 9}$ & $\mathbf{2 0 0 0}$ & $\mathbf{2 0 0 1}$ \\
Ansprache durch & $\mathbf{\%}$ & $\mathbf{\%}$ & $\mathbf{\%}$ & $\mathbf{\%}$ \\
\hline Extern: & & & & \\
$\quad$ - frühere Kunden/Nachfolgeprojekte & - & - & 20 & 10 \\
$\quad$ - Netzwerkspartner/Kooperationspartner & - & - & 5 & 7 \\
$\quad$ - Referenzen/Empfehlungen & - & 7 & 10 & 6 \\
$\quad$ - Veranstaltungen & - & 10 & 4 & 18 \\
$\quad$ - Homepage & - & 2 & 15 & 7 \\
$\quad$ - direkter Kontakt/keine Angaben & 50 & 20 & 11 & 27 \\
Intern: & & & 35 & 25 \\
$\quad$ - andere Abteilungen (Innovationsagentur) & 50 & 60 & $100 \%$ & $100 \%$ \\
Summe & $100 \%$ & $100 \%$ & Schätzungen/Werte gerundet
\end{tabular}

Marketing und Projektakquirierung liegen in der Verantwortung der einzelnen Bereichsleiter. Bis Mai 2002 gab bei der Innovationsagentur keine zentral verantwortliche Person für Marketing und keine zentral geführten, allgemein zugänglichen Aufzeichnungen über Veranstaltungen, potentielle Kooperationspartner, Medien, etc. Daraus resultiert ein relativ hoher Marketingaufwand für jeden einzelnen Geschäftsbereich. 1999 
wurden, um den Marketingaufwand möglichst gering zu halten, ein großer Teil der extern verrechneten Projekte durch den Geschäftsbereich SeedCapital vermittelt. 2001 war ein höherer Marketingaufwand erforderlich - durch die allgemein schlechte wirtschaftliche Lage der Finanz- und IT-Industrie und durch große Programmunsicherheiten bei den anderen Geschäftsbereichen der Innovationsagentur.

\subsubsection{Leistungserbringung}

Der Entwurf des Informationsmaterials (Image-Folder) ist professionell gelayoutet und verspricht die Dienstleistungen des TecNet ansprechend zu vermitteln. In den ,success stories" finden sich Großunternehmen wie die Siemens AG, kleine und mittlere Firmen wie die Ikarus Software GmbH oder junge Unternehmen wie die Funktionswerkstoffe F\&E GmbH. Diese Referenzen zeigen, dass zwar technologieintensive Unternehmen im Vordergrund stehen, das TecNet sich jedoch nicht auf bestimmte große oder kleine Unternehmen(-sgruppen) konzentriert. Ein Konzept für eine gezielte PR- und Medienarbeit liegt bislang nicht vor bzw. ist nicht erkennbar, befindet sich nach Auskunft von TecNet aber im Aufbau. In den Jahresberichten 2000 und 2001 werden folgende Aktivitäten unter die Rubrik Öffentlichkeitsarbeit/Marketing genannt:

Präsentation von TecNet (Vorträge)

- im Rahmen einer WiFi-Veranstaltung (2000),

- bei einer Vortragsreihe „Innovation lebt von Information“ (2000),

- einem BAWAG-Schulungs-Seminar (2000),

- beim achten $\mathrm{i}^{2}$-Forum (2000),

- für die BÜRGES Förderungsbank (2000)

- ZAT/Zentrum für angewandte Technologie, Leoben (2001)

- für die BÜRGES Förderungsbank (2001)

- für neue Mitarbeiter der Innovationsagentur (2001).

Folgt man der strengen Definition eines Marketing, als „Unternehmenskonzeption, bei der im Interesse der Erreichung der Unternehmensziele alle betrieblichen Aktivitäten konsequent auf die gegenwärtigen und zukünftigen Erfordernisse der Märkte bzw. der Abnehmer (Kundenorientierung) ausgerichtet werden“, so ist eine externe Kundenorientierung nicht offensichtlich. Sowohl das WiFi als auch die BÜRGES Förderungsbank sind Gesellschafter der Innovationsagentur. Das i ${ }^{2}$-Forum ist ebenfalls eine Aktivität der Innovationsagentur und interne Vorträge vor neuen Mitarbeitern sind keine kundenorientierten Werbemaßnahmen im eigentlichen Sinne. 


\subsubsection{Preisstrategie}

Das TecNet berechnet seine Leistung nach Aufwand gemäß der Formel:

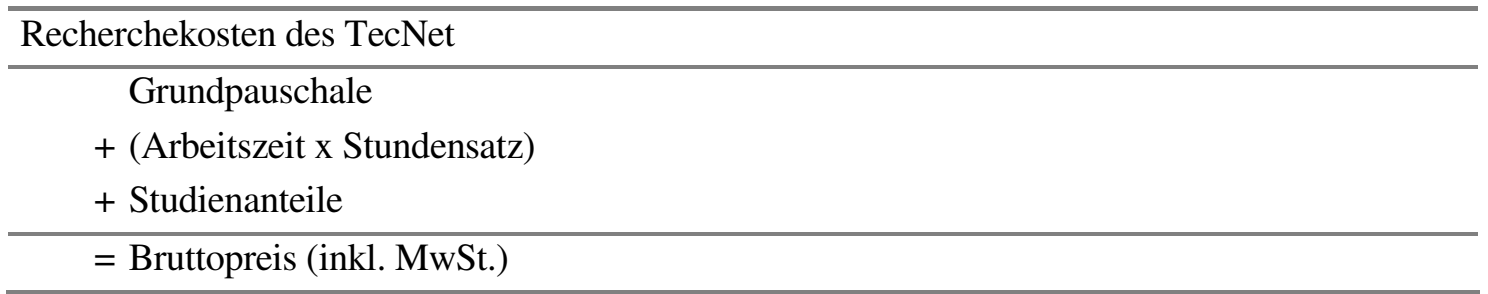

Die Grundpauschale in Höhe von $€ 181,68$ fällt nur bei externen Aufträgen an. Der Aufwand bemisst sich für reine Datenbankrecherchen auf eine Dauer von zwei bis acht Arbeitsstunden. Umfangreichere Recherchen werden mit einem Arbeitsaufwand von ca. 30 bis 80 Stunden (Suche in Datenbanken, Internet, Expertenanfragen etc. und Endbericht) angegeben (Materialien 13, S. 1, Punkt 3). Die Kosten bzw. Arbeitsstunden des TecNet werden wie folgt verrechnet (Materialien 15, S. 2):

- Kosten der Recherche/Studienkosten

Die Grundpauschale für den Datenbankzugang sowie für die Entwicklung einer Suchstrategie beträgt $€ 181,68$. Der von TecNet angesetzte Stundensatz beträgt für die weitere Arbeiten $€$ 90,84. Die externen Kosten der Markt- und/oder Technologiestudien bzw. der Studienauszüge beträgt je nach Quelle zwischen $€ 14,53$ und $€ 2.906,91$.

- Kosten der Vermittlung von Expertengesprächen/Gutachtern

Die Bearbeitungskosten sind hier mit einer Grundpauschale von $€ 181,68$ sowie einem Stundensatz von $€ 90,84$ angesetzt. Zu diesen Kosten trägt der Kunde die Honorare der angefragten Experten, die für Gespräche bzw. Gutachten zu Marktund/oder Technologiefragen vermittelt werden.

Die Preise wurden nach anfallenden Kosten und nach einer vermuteten „Marktakzeptanz“ gestaltet. Das TecNet verfolgt keine „Low- oder High-price“-Strategie sondern bietet im Mittelfeld, d.h. zwischen den üblichen Beraterhonoraren und den nahezu oder kostenlosen Informationsdiensten anderer öffentlicher Einrichtungen an. $\mathrm{Zu}$ diesen zählen z.B. Techinform/WiFi, einzelne Landesstellen der Wirtschaftskammer (Tirol, NÖ, OÖ), ARC Seibersdorf, Bereuter (Informationsbroker). Die Preisgebung der TecNet-Dienstleistung hat die Funktion die Ausgaben der Gesellschaft zumindest teilweise zu decken, sowie seriöse Anfragen sicherzustellen. Ferner wird durch die Preisgebung erreicht, dass der kostenbewusste Kunde von vornherein seine Fragestellung bzw. seine Wünsche fokussiert - und es nicht zu einem diffusen Auftragsverhältnis kommt. Der Instrumentalisierung durch kommerzielle Beratungsgesellschaften oder unseriöser Auftraggeber wird auf diese Weise entgegengewirkt. 


\subsection{AUFTRAGSLAGE UND FINANZIERUNG}

\subsubsection{Kundengruppen}

Grundsätzlich lassen sich zwei Kundengruppen des TecNet unterscheiden: Etwa die Hälfte aller Projekte finden sich in den TecNet benachbarten Organisationseinheiten der Innovationsagentur (TecMa, SeedCapital). Die andere Hälfte aller Projekte erfolgt durch externe Auftraggeber (Unternehmen, Erfinder etc.).

Innerhalb der Innovationsagentur verteilen sich rund $80 \%$ der Projekte auf den internen „Kunden“ TecMa, die übrigen $20 \%$ finden sich bei SeedCapital. Hier eröffnet sich dem Evaluationsteam das Problem, dass es über kein Mandat verfügt, die Kundengruppen dieser Bereiche zu prüfen, da diese Bereiche nicht Bestandteil der Evaluation sind. Aussagen zu den „Kunden der Kunden“ sind daher nur sehr eingeschränkt möglich. Die vorliegenden Zahlen beschränken sich auf anonymisierte Angaben von TecNet im Rahmen der Projekte sowie auf die veröffentlichen Success-Stories.

In Tabelle 2 wurde die Rechtsform der Kundengruppen des TecNet für die Jahre 2000 und 2001 nach ihren Ertragswerten unterschieden. Insgesamt dominieren GmbHs und Aktiengesellschaften das Gesamtbild. Im Jahr 2001 entfällt erstmals knapp ein Drittel der Erträge auf Universitäten, internationale Organisationen und andere Einrichtungen. In dieser Auflistung verwundert, dass in 2001 keine Privatperson, GbR mehr zu finden ist, obwohl gerade hier eine wichtige Zielgruppe des TecNet, die der Unternehmensgründer und Erfinder, zu vermuten sind. Andererseits ist es nicht ungewöhnlich, dass BusinessPläne, Finanzierungs- und Förderanträge nur von Unternehmen (i.d.R. GmbHs), nicht aber von Privatpersonen erstellt bzw. eingereicht werden dürfen, so dass der „Gründungsakt" vor der Kontaktaufnahme mit dem TecNet stand.

Tabelle 2: $\quad$ Erträge externer Kundengruppen nach Rechtsform

\begin{tabular}{lcc}
\hline $\begin{array}{l}\text { Verteilung der Erträge } \\
\text { bei externen Kunden }\end{array}$ & $\begin{array}{c}\mathbf{2 0 0 0} \\
\mathbf{\%}\end{array}$ & $\begin{array}{c}\mathbf{2 0 0 1} \\
\mathbf{\%}\end{array}$ \\
\hline Privatpersonen, GbR & 3 & 0 \\
Ges.m.b.H., AG & 94 & 71 \\
Universitäten & 3 & 11 \\
Internationale Organisationen & 0 & 11 \\
Andere ……………………….... & 0 & 7 \\
\hline Summe & 100 & 100 \\
\hline
\end{tabular}

TecNet Angaben gemäß Evaluationsunterlagen/Gespräche

Im Projektportfolio des TecNet finden sich überwiegend kleine und mittlere High-TechUnternehmen, die jedoch nicht immer als „Start-Ups“ einzustufen sind. Ein Jungunternehmen wird als Firma mit einem Alter von bis zu drei Jahren definiert.

Im Zeitverlauf (vgl. dazu Abbildung 2) ist festzustellen, dass TecNet seine Tätigkeit stärker auf Jungunternehmen und Erfinder ausrichtet und der Anteil etablierter Firmen als Kundengruppe kontinuierlich abnimmt. Die wichtige Zielgruppe der Kapitalgeber wird 
nach dem Boomjahr 2000 (20 \% aller Projekte) in 2001 leider nicht mehr so oft erreicht. Hier finden sich aktuell noch sechs Projekte, wobei sich die Anzahl der Kunden durch mehrfache Projektbeauftragung z.B. durch die BÜRGES-Förderbank auf ein bis zwei Kunden reduziert. Angesichts der Zielsetzung verschiedene Technologie-Financiers, d.h. Kreditinstitute, Förderbanken, Business-Angles und Venture-Capital Gesellschaften mit dem Angebot des TecNet zu erreichen, ist diese Resultat vergleichsweise unbefriedigend.

Abbildung 2: TecNet-Projekte nach Kundengruppen

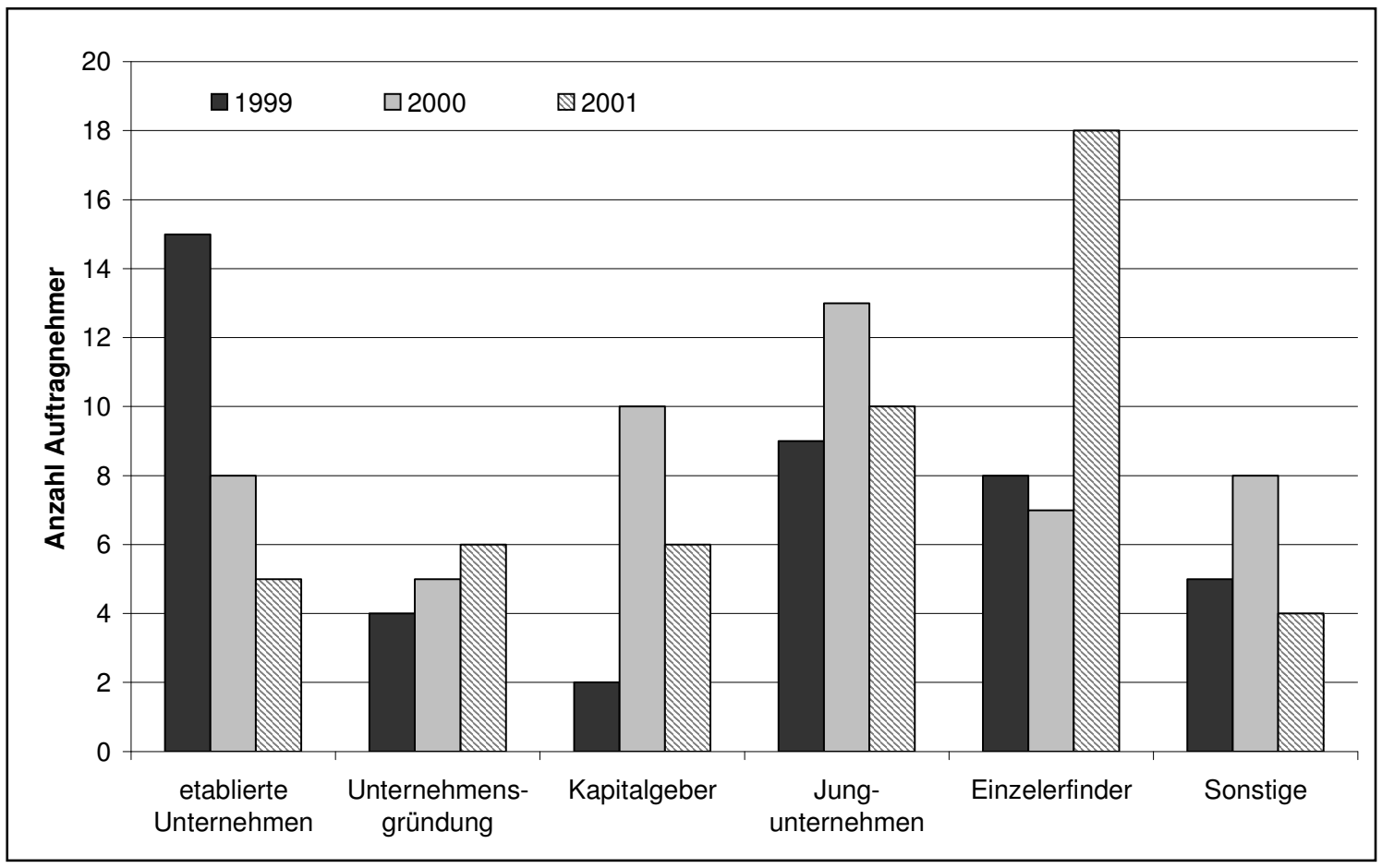

TecNet Angaben gemäß Evaluationsunterlagen/Gespräche

Im Gesamtbild machen die Projekte der High-Tech Unternehmensgründer über die Jahre einen Kundenanteil von rund elf Prozent aus, bei Jungunternehmen beträgt der Anteil zwischen $20 \%$ und $25 \%$. Stark angestiegen ist das Projektaufkommen durch den internen Kunden TecMa an das TecNet. Das TecMa zählt inzwischen mit Abstand zu den bedeutendsten Kunden des TecNet. Während die Projekte der „Erfinder“ im Jahr 2000 noch $14 \%$ aller Projekte ausmachten ist diese Zahl in 2001 auf $38 \%$ angewachsen (vgl. auch Tabelle 3).

\subsubsection{Projekte}

Seit dem Jahr 2000 werden jährlich zwischen 80 und 100 Anfragen an das TecNet gerichtet, wobei rund die Hälfte der Anfragen bearbeitet wurden, aber nach Erstellung des projektbezogenen Angebots zu keinem Auftrag führten (Materialien 12 und Materialien 13, S. 1, Punkt 2.). Das TecNet führt jährlich rund 50 Projekte unterschiedlicher Größenordnung durch. Diese Projekte werden, je nach Auftraggeber, innerhalb der Innovationsagentur ,,intern“ verrechnet oder ,externen“ Kunden in Rechnung gestellt. Eine Ausnahme stellen Projekte für die BÜRGES Förderungsbank dar. Da die BÜRGES Förderungsbank räumlich, inhaltlich, gesellschaftsrechtlich und personell unmittelbar mit 
der Innovationsagentur verbunden ist, werden BÜRGES Aufträge zwar wie externe Projekte durchgeführt, jedoch wie interne Projekte ohne Grundpauschale verrechnet. ${ }^{2}$

Im Zeitverlauf ist festzustellen, dass jährlich zwischen vier und acht Projekte ohne Leistungsverrechnung (Demonstrationsprojekte, Marketingaktivitäten für Referenzkunden) durchgeführt werden. Gleichzeitig ist zu beobachten, dass der Anteil Projekte mit interner Leistungsverrechnung in 2001 nach einem ausgeglichenen intern/externVerhältnis der Vorjahre überwiegt.

Tabelle 3: $\quad$ Anzahl interner/externer Projekte 1998-2001

\begin{tabular}{|c|c|c|c|c|c|c|c|c|}
\hline & \multicolumn{2}{|c|}{1998} & \multicolumn{2}{|c|}{$1999 *$} & \multicolumn{2}{|c|}{2000} & \multicolumn{2}{|c|}{ 2001* } \\
\hline & abs. & $\%$ & abs. & $\%$ & abs. & $\%$ & abs. & $\%$ \\
\hline Anzahl Projektanfragen & $-^{* *}$ & & ca. 60 & & 81 & & 99 & \\
\hline Anzahl durchgeführte Projekte & 28 & 100 & 41 & 100 & 50 & 100 & 50 & 100 \\
\hline ... davon interne Verrechung & 2 & 7 & 11 & 27 & 17 & 34 & 23 & 46 \\
\hline ... intern ohne Leistungsverrechnung & 15 & 54 & 8 & 20 & 2 & 4 & 3 & 6 \\
\hline ... davon externe Verrechnung & 3 & 11 & 18 & 43 & 29 & 58 & 17 & 34 \\
\hline ... extern ohne Leistungsverrechnung & 8 & 29 & 4 & 10 & 2 & 4 & 7 & 14 \\
\hline Anzahl externer Kunden & 10 & & 20 & & 25 & & 18 & \\
\hline
\end{tabular}

* Projekte für die BÜRGES Förderungsbank werden als externe Projekte gewertet, da es sich bei BÜRGES und der Innovationsagentur GmbH formal um zwei eigenständige Unternehmen handelt. Dennoch wird bei Aufträgen durch die BÜRGES Förderungsbank keine Grundpauschale, wie sonst üblich, erhoben.

** Daten nicht recherchierbar.

Grundsätzlich ist anzumerken, dass die Zahl der Projekte nicht mit der Anzahl unterschiedlicher Auftraggeber (Unternehmen) gleichgesetzt werden darf. Insgesamt entfallen im Jahr 2000 (2001) auf 25 (18) unterschiedliche Unternehmen 31 (24) Projekte. Dieses Verhältnis erklärt sich daraus, dass einige Unternehmen wiederholt bzw. mehrere Projekte bei TecNet in Auftrag gegeben haben. TecNet bediente zwischen 1998 und 2001 insgesamt 73 mal die Privatwirtschaft. Es ist jedoch auch hierbei zu beachten, dass einige Unternehmen das TecNet nach ca. einem Jahr erneut beauftragt haben (z.B. BÜRGES Förderungsbank). Ohne Doppelzählungen hat TecNet im gesamten Bestandszeitraum für 65 unterschiedliche Unternehmen gearbeitet.

\subsubsection{Auftragsvolumen}

In der Finanzierungsstruktur der Projekte ist erkennbar, dass nach den Jahren 1999 und 2000 die externen Einnahmen in 2001 deutlich zurückgegangen sind. Wurden 1999/2000 noch rund $€ 17.000$ durch externe Aufträge (TecNet-Aufwand) umgesetzt, so sind es 2001 nur noch rund $€ 10.000$. Demgegenüber hat sich die interne Auftragsarbeit von rund $€ 3.700$ auf rund $€ 12.100$ mehr als verdreifacht. Der Großteil dieser Aufträge wurde von der Schwesterorganisation TecMa vergeben.

\footnotetext{
${ }^{2}$ Bei der Zuordnung der BÜRGES Förderungsbank besteht seitens des TecNet die Unsicherheit ob man die Bank als „interne“ oder als „externe“Einrichtung in den Büchern führt.
} 
Tabelle 4: Internelexterne Projekteinnahmen 1998-2001

\begin{tabular}{|c|c|c|c|c|c|c|c|c|c|}
\hline & \multicolumn{2}{|c|}{1998} & \multicolumn{2}{|c|}{1999} & \multicolumn{2}{|c|}{2000} & \multicolumn{2}{|c|}{2001} & \multirow{2}{*}{$\begin{array}{r}\text { Summe } \\
E U R\end{array}$} \\
\hline & EUR & $\%$ & EUR & $\%$ & EUR & $\%$ & EUR & $\%$ & \\
\hline Projekteinnahmen (Summe in $€$ ) & 2.180 & 100 & 22.973 & 100 & 35.470 & 100 & 25.491 & 100 & \\
\hline ... davon intern (TecNet-Aufwand) & & & 1.908 & 8 & 3.747 & 11 & 12.105 & 47 & 18.849 \\
\hline ... davon intern (Studienanteile etc.) & 1.090 & 50 & 0 & 0 & 5.324 & 15 & 605 & 2 & 5.929 \\
\hline ... davon extern (TecNet-Aufwand) & & & 16.438 & 72 & 17.575 & 50 & 9.889 & 39 & 44.991 \\
\hline ... davon extern (Studienanteile etc.) & 1.090 & 50 & 4.628 & 20 & 8.824 & 25 & 2.893 & 11 & 16.345 \\
\hline
\end{tabular}

Setzt man diese Zahlen in Relation zu den Projekten, dann wurden im Jahr 2000 durchschnittlich $€ 197,00$ pro internes Projekt verrechnet, während dieser Betrag im Jahr 2001 auf durchschnittlich $€ 466,00$ je internes Projekt angestiegen ist. Da es sich um verrechnete Arbeitsstunden handelt, ist der interne Arbeitsaufwand überproportional angestiegen, während der Aufwand für externe Projekte von durchschnittlich 567,00 $€ /$ Projekt auf 412,00 €/Projekt gesunken ist. Diese Fakten lassen verschiedene Schlüsse $\mathrm{zu}:$

- Die „willingness to pay“ der internen Kunden scheint höher als erwartet zu sein;

- Die Diskrepanz der internen Projekteinnahmen (TecNet-Aufwand und Studienanteile) erklärt sich u.a. aus einzelnen Großprojekten die belegen, dass ein standardisierter Rechercheablauf noch nicht vorherrscht.

- Es steht zu vermuten an, dass wegen des hohen Arbeits- und Akquisitionsaufwands bei gegebenen Ressourcen (Personal) die internen Projekte bevorzugt werden.

\subsubsection{Arbeitsstunden}

In Tabelle 5 wurden die intern und extern verrechneten Erträge des TecNet durch den verrechneten Arbeitsstundensatz (90,98 EURO) dividiert, um einen Überblick über die Produktivität zu erhalten.

Es zeigt sich, dass von jährlich etwa 1.800 Arbeitsstunden zwischen 1999 und 2001 im Mittel lediglich rund 143 Stunden extern (ca. $8 \%$ ) und etwa 67 Stunden intern (ca. $4 \%$ ) mit einem Preis bewertet werden. Insgesamt können im durchschnitt 675 Stunden pro Jahr konkreten, internen oder externen Projekten zugerechnet werden. Die übrigen Arbeitsstunden fallen für Verwaltungsaufgaben, Vorträge, Weiterbildung, Marketing und andere Aktivitäten an. $37 \%$ der Arbeitszeit entfiel also im Durchschnitt über die Jahre auf konkrete Projektarbeit, $63 \%$ auf Verwaltungsaufgaben.

Ähnlich das Verhältnis zwischen verrechneten und nicht verrechneten Projektarbeitsstunden: Während 68\% der Zeit, in der konkret auf Projekten gearbeitet wurde, nicht weiterverrechnet wurde, sind nur $32 \%$ der Projektarbeitszeit gem. Aufwand entschädigt worden.

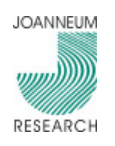


Nicht eingerechnet wurde in diese Kalkulation die regelmäßige Assistenz für Bibliothek und administrative Aufgaben sowie die Projektassistenz. Ferner wurden in den Anfangsjahren 1998/1999 geschätzte 50 Überstunden p.a. geleistet, die sich in den Boomjahren technologieintensiver Unternehmensgründungen 2000 und 2001 auf mehr als 200 Überstunden p.a. steigerten. Aufgrund des persönlichen Engagements der Bereichsleiterinnen wird die effektive Anzahl der geleisteten Überstunden jedoch noch deutlich höher eingeschätzt. Es zeigt sich, dass zahlreiche Aufgaben von der Bereichsleitung selbst wahrgenommen werden müssen und es nur wenige Möglichkeiten gibt, Arbeitsaufgaben zu delegieren. Die Projektassistenz ist zwar bei internen Arbeiten (Verwaltung, Sekretariatsdienste etc.) ein Hilfe, die Akquisition, die Fachgespräche mit Kunden, Vorträge etc. verbleiben jedoch in den Arbeitsstunden bei der Bereichsleitung.

Das heißt der Kostendeckungsgrad ist allein in Bezug auf die Arbeitskosten der Bereichsleitung mit durchschnittlich $12 \%$ sehr gering (Bei Berücksichtigung der Gemeinkosten der Innovationsagentur für Miete etc. in dieser Kalkulation würde der Kostendeckungsgrad noch zusätzlich sinken).

\subsubsection{Kontrollinstanzen}

Die von TecNet erwirtschafteten Erträge sowie die angefallenen Kosten werden innerhalb der Innovationsagentur (Verwaltung) und vom BMWA regelmäßig geprüft. Darüber hinaus werden Tätigkeitsberichte (inkl. Kosten-/Ertragslage) quartalsweise an das BMWA und den Aufsichtsrat der Innovationsagentur übermittelt, um den Berichts- und Kontrollpflichten zu genügen. Die Kunden gewinnen anhand der individuellen Recherchedokumentation einen Einblick in den vom TecNet betriebenen Aufwand (Datum, Uhrzeit, Tätigkeit, Person, Ergebnisse). 
Tabelle 5: $\quad$ Arbeitsstunden der Bereichsleitung 1999-2001

\begin{tabular}{|c|c|c|c|c|c|c|}
\hline & 1999 & in $\%$ & 2000 & in $\%$ & 2001 & in $\%$ \\
\hline TecNet-Einnahmen ohne Studienzukauf [€] & 18.168 & & 21.438 & & 21.802 & \\
\hline Arbeitstage (abzgl. 30 T Urlaub, 7 T. Krankheit) & 210 & & 210 & & 210 & \\
\hline Arbeitsstunden pro Tag (38,5 h/Woche) & 7,7 & & 7,7 & & 7,7 & \\
\hline Arbeitsstunden/Jahr (38,5 h/Woche $)^{\mathrm{a}}$ & 1.617 & & 1.617 & & 1.617 & \\
\hline + Überstunden & 50 & & 200 & & 200 & \\
\hline = Summe Arbeitsstunden p.a. & 1.667 & & 1.867 & & 1.867 & \\
\hline $\begin{array}{l}\text { Arbeitsstunden für konkrete Anfragen } \\
\text { Aufwand nicht verrechnet }\end{array}$ & 30 & 4 & 90 & 13 & 150 & 25 \\
\hline $\begin{array}{l}+ \text { Arbeitsstunden für externe Projekte } \\
\text { Aufwand verrechnet }\end{array}$ & 165 & 23 & 170 & 24 & 95 & 16 \\
\hline $\begin{array}{l}+ \text { Arbeitsstunden für externe Projekte } \\
\text { Aufwand nicht verrechnet } \\
\text { (z.B. Vorrecherche, Referenzprojekte, Testprojekte) }\end{array}$ & 250 & 35 & 380 & 53 & 170 & 29 \\
\hline $\begin{array}{l}+ \text { Arbeitsstunden für interne Projekte } \\
\text { Aufwand verrechnet }\end{array}$ & 25 & 4 & 40 & 6 & 135 & 23 \\
\hline $\begin{array}{l}\text { Arbeitsstunden für interne Projekte } \\
\text { Aufwand nicht verrechnet } \\
\text { (z.B. Referenzprojekte, interne Testprojekte) }\end{array}$ & 240 & 34 & 40 & 6 & 45 & 8 \\
\hline Summe Projekte + Anfragen [1] & 710 & 100 & 720 & 100 & 595 & 100 \\
\hline $\begin{array}{l}\text { Marketing von TecNet } \\
\text { (z.B. Marketingkonzept, Homepage, Folder, Vorträge) }\end{array}$ & 200 & 21 & 220 & 19 & 320 & 25 \\
\hline $\begin{array}{l}\text { + Vorträge/Fachveranstaltungen/Reisen } \\
\text { (z.B. Vorträge Fachveranstaltungen; Organisation } \\
\text { Veranstaltungsreihe „Innovation lebt von Information“) }\end{array}$ & 100 & 10 & 150 & 13 & 120 & 9 \\
\hline $\begin{array}{l}\text { Planung/Strategie TecNet } \\
\text { (z.B. Suchen/Testen neue Informationsquellen; } \\
\text { Vertragsverhandlungen mit Informationsanbietern; } \\
\text { Gespräche mit potenziellen Kooperationspartnern) }\end{array}$ & 250 & 26 & 280 & 24 & 250 & 20 \\
\hline $\begin{array}{l}\text { + externe und interne Kommunikation/ } \\
\text { Organisation/Management } \\
\text { (z.B. Bereichsleiterbesprechungen, Frühbesprechungen } \\
\text { mit Assistentin; Zieldefinition mit Geschäftsführung, } \\
\text { Mitarbeitergespräch mit Assistentin; Budgeterstellung, } \\
\text { Bereichscontrolling; Statistiken und Fragebögen für } \\
\text { Ministerien, etc.; ... Beantworten diverser allgemeiner } \\
\text { Anfragen nach Förderstellen; Mail, Post) }\end{array}$ & 250 & 26 & 300 & 26 & 300 & 24 \\
\hline $\begin{array}{l}+\quad \text { Verwaltungsaufgaben }^{\mathrm{b}} \\
\quad \text { (z.B. Berichte für Ministerien und Aufsichtsrat) }\end{array}$ & 54 & 6 & 54 & 5 & 54 & 4 \\
\hline $\begin{array}{l}\text { + Weiterbildung } \\
\text { (z.B. Seminare, Einschulung durch Vorgängerin, } \\
\text { Schulungen durch Datenbankanbieter) }\end{array}$ & 50 & 5 & 90 & 8 & 30 & 2 \\
\hline $\begin{array}{l}\text { + Sonstiges } \\
\text { (z.B. Arbeiten verbunden mit der Einführung eines } \\
\text { Work-Flow-Systems in der IA (Notes); Ablage, } \\
\text { Störungsbehebung von Datenbankanbindungen) }\end{array}$ & 53 & 6 & 53 & 5 & 198 & 16 \\
\hline$=$ Summe Arbeitsstunden p.a. [2] & 957 & 100 & 1.147 & 100 & 1.272 & 100 \\
\hline Saldo $[1]+[2]$ & 1.667 & 100 & 1.867 & 100 & 1.867 & 100 \\
\hline
\end{tabular}

Anmerkung: Die Arbeitsstunden sind z.T. geschätzt und wurden auf fünf Stunden gerundet. 


\subsubsection{Technologiefelder}

Eine Aufschlüsselung der von TecNet abgeschlossenen Projekte nach Technologiefeldern zeigt, dass die Informations- und Kommunikationstechnologien bis ins Jahr 2000 den dominierenden Anteil ausmachen. In 2001 wurden überwiegend Biotechnologie-Projekte bearbeitet (34 \%), gefolgt von Projekten aus dem Bereich der Physik (27 \%) und - immer noch stark vertreten - der IuK-Technolgien (24\%).

Abbildung 3: Abgeschlossene Projekte nach Technologiefeldern 1998-2001

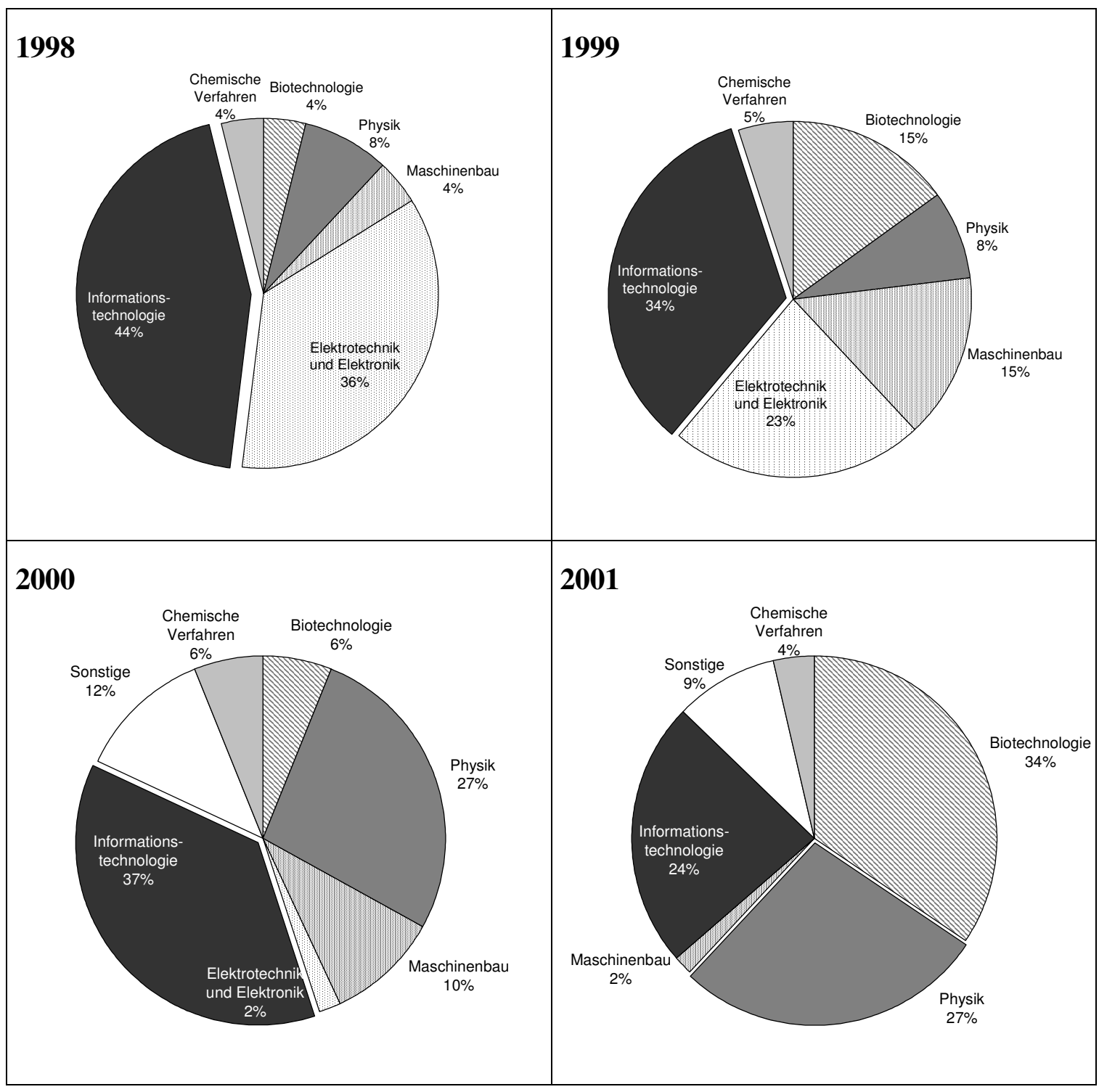

Die Auftragsverteilung in diesen „modernen Technologiezweigen“ ist als Indiz zu werten, dass alle Kunden des TecNet entweder in High-Tech Branchen beheimatet sind oder dass es sich um Kapitalgeber handelt, die Finanzierungsentscheidungen von Marktrecherchen abhängig machen. 


\subsection{AUFTRAGSABWICKLUNG UND ARBEITSPROZESSE}

\subsubsection{Projekt- und Arbeitsablauf}

Die Auftragsabwicklung im TecNet lässt sich wie folgt beschreiben:

1. Kundenanfrage (telefonisch, email oder persönlich)

2. Führen eines Vorgesprächs/Vorabinformationen

3. Präsentation des Projekts und Besprechung der Fragestellung

4. Rückfragen von TecNet (telefonisch oder via email)

5. Präzisierung der Fragestellung durch TecNet und Kunden

6. Unverbindliches Angebot (Stundensätze, Kosten für Studienteile)

7. Vorrecherche

8. Projektangebot

9. Auftragserteilung

10. Durchführung der Recherche (Delegieren an Projektassistenz)

11. Zwischenergebnisse

12. Abstimmung mit Kunden (Rechercheerfolg, zusätzliche Recherchen/Studien)

13. Endbericht

14. Rechnungserstellung

Das TecNet schätzt den Beratungsaufwand für die Mandanten vor Vertragsabschluß (Punkt 5) als gering ein. Gründerpersönlichkeiten, Start-Up Firmen und wachsende Unternehmen benötigen kaum eine zusätzliche Beratungsleistung durch das TecNet. Überwiegend verständlich und präzise sind auch die Anfragen von etablierten Gesellschaften, so dass hier nur eine geringe Beratung erforderlich ist. Hingegen muss sich TecNet den Fragen und Vorstellungen von privaten Erfindern in umfangreicherer Form widmen. Hier ist der Beratungsaufwand vor Vertragsabschluss zu 60 Prozent vergleichsweise hoch.

Im Bearbeitungsaufwand gibt das TecNet zwei Schwerpunkte an: Einerseits werden Datenbankrecherchen mit einer Dauer von zwei bis acht Stunden durchgeführt, andererseits erfordern umfangreiche Recherchen einen Arbeitsaufwand von ca. 30 bis 80 Stunden (Materialien 13, S. 1, Punkt 3). Dabei ist jedoch unklar, ob dieser Aufwand die Arbeit der Vorrecherchen (Auftragsanbahnung) einschließt oder nicht. Der prinzipielle Ablauf eines Projekts ist in Abbildung 4 skizziert. In diesem Ablaufdiagramm fällt auf, dass einem Kunden vor einer Vertragsvereinbarung vier Exit-Optionen offen stehen: Zum einen kann ein Auftrag scheitern, weil die Dienstleistungen des TecNet falsch eingeschätzt wurden [1]. Im Anschluss eines Vorgesprächs kann es dann zu unterschiedlichen Erwartungshaltungen kommen, die den Kunden Abstand vom Auftrag nehmen lassen [2]. Die Vorrecherche kann zu mangelnden Ergebnissen führen, oder den 
Kunden dazu bewegen selbständig weiter zu recherchieren [3]. Ferner ist es möglich, dass das Projektangebot dem Kunden zu teuer oder zu wenig erfolgversprechend für seine Bedürfnisse erscheint [4]. Für das TecNet sind Auftraganbahnungen daher immer mit hoher Unsicherheit behaftet.

\section{Abbildung 4: Projektabwicklung des TecNet im Flussdiagramm}

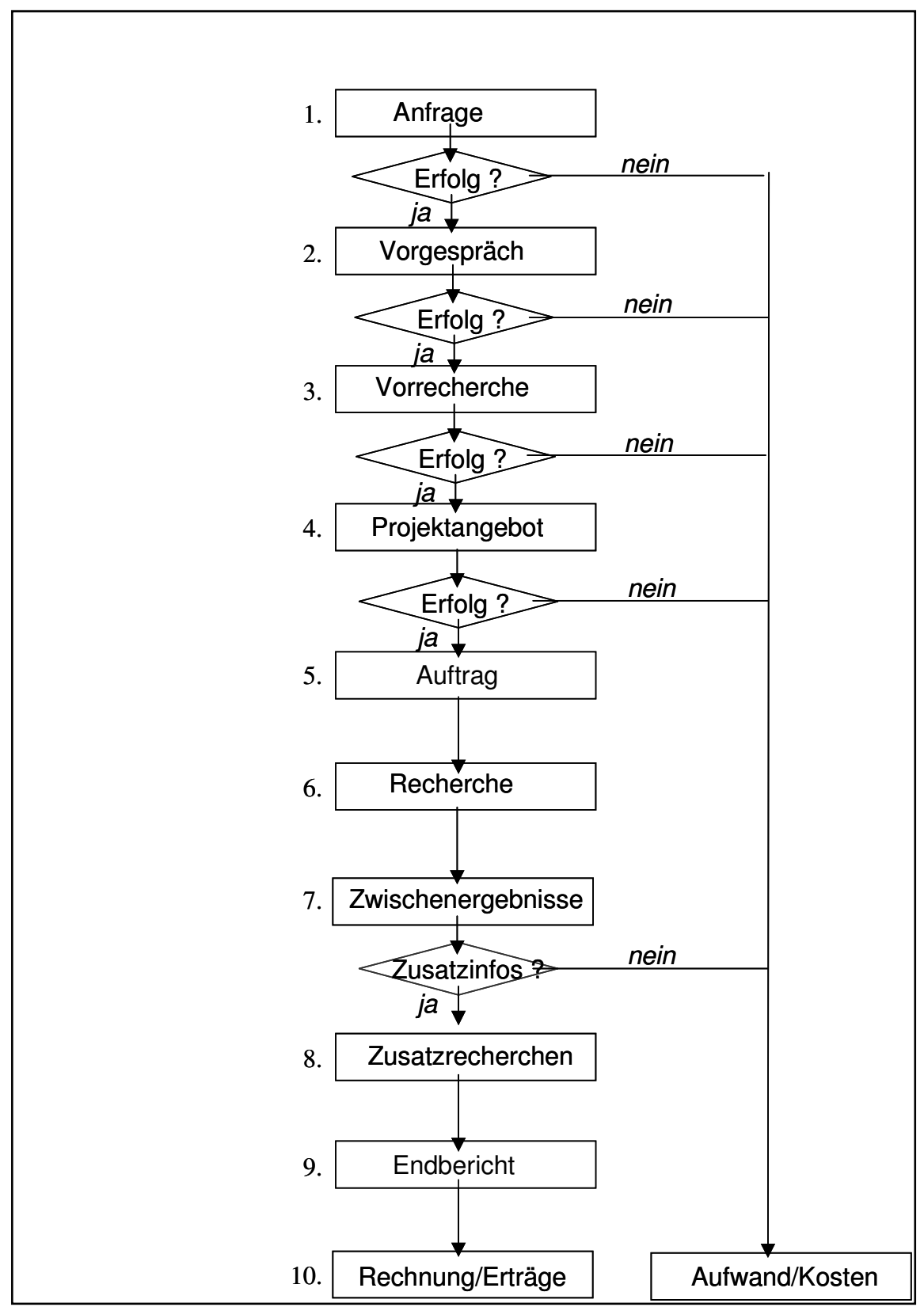

\subsubsection{Qualifikationsanforderungen}

Für die Recherche in technologieintensiven Wirtschaftszweigen ist eine ingenieurwissenschaftliche Qualifikation eine wichtige Voraussetzung. Der technische Hintergrund erleichtert die Verständigung und das Verständnis über den $\mathrm{zu}$ recherchierenden Sachverhalt. Da ein Informationsbroker im Internet und mit Datenbanken weltweit agiert, sind sehr gute englische und nach Möglichkeit Sprachkenntnisse in weiteren Sprachen 
von Vorteil. Darüber hinaus muss ein Informationsvermittler Geduld mitbringen und über ein ausgeprägtes strategisches und analytisches Denkvermögen verfügen. Frau DI Bauer wird als Bereichsleiterin von TecNet diesen Anforderungen voll gerecht.

\subsubsection{Arbeitsprozesse}

$\mathrm{Zu}$ den wichtigsten Arbeitswerkzeugen von Informationsbrokern zählen ein Telefonanschluss, ein leistungsfähiger PC sowie ein Internetzugang. Mit diesen Arbeitsmitteln, aber auch mit Erfahrung und persönlichen Kontakten, sichert sich das TecNet den Zugang zu Informationsquellen weltweit. Es werden Datenbanken abgefragt, aktuelle Informationen gesammelt, gebündelt und bewertet. TecNet recherchiert überwiegend „online“, aber auch mittels zugekaufter Datenträger sowie über Printmedien. Die Handelsware bzw. das Produkt ist das Wissen, das durch Fachkompetenz und die Fähigkeit Netzwerke zielgerichtet $\mathrm{zu}$ nutzen mit jedem Auftrag erweitert wird. Durch die umfangreiche Recherche $\mathrm{zu}$ bestimmten Themen generiert TecNet mit jedem Projekt neues, komprimiertes Wissen.

Vor einer Recherche wird eine Suchstrategie entwickelt und mit zunehmender Erfahrung erkennt ein erfahrener Rechercheur immer schneller, wo welche Information zu suchen sind, wie diese strukturiert sind und welche Schlüsselworte bei einer Suche Aussicht auf eine überschaubare Trefferzahl bietet. Auf diese Weise werden Lern- und Skaleneffekte erzeugt, die dem TecNet eine höhere Produktivität bei der Informationssuche gewährleisten, als sie von Unternehmen bzw. Personen erzielt werden können, die nur gelegentlich in Datenbanken oder im Internet recherchieren.

Der Arbeitsprozess sollte einerseits standardisiert sei, was die Vorrecherchen, das Projektangebot, die Projektdurchführung sowie den Projektabschluss betrifft. Andererseits muss ein Informationsbroker aber immer auch mit Kreativität und Sachverstand nach Informationen suchen und sich dem rasanten Wandel bei elektronischen Medien (z.B. bei Menüführung, Abfrage-Prozeduren, Installationen, Zugriffskontrollen etc.) anpassen.

\subsubsection{Feedback und Kundenzufriedenheit}

Zur Beurteilung der Arbeit und der Qualität der gelieferten Informationen ist ein Feedback bzw. ist die Kundenzufriedenheit ein wichtiger Indikator. Das TecNet führt zwar keine eigene Leistungsbewertungen durch, erfährt jedoch unterschiedliche Hinweise (persönliche Ansprache, schriftlich, mündlich) auf die Zufriedenheit seiner Kunden. Positiv hervorgehoben wird nach Aussage des TecNet, dass die Zusammenfassungen der Ergebnisse in den Endberichten gelungen ist, das die Rechercheergebnisse einen guten Marktüberblick vermitteln und das Informationen recherchiert wurden, auf die insbesondere kleine Unternehmen für gewöhnlich keinen Zugang finden. Die Organisationseinheiten innerhalb der Innovationsagentur wünschen sich - natürlich - einen kostenlosen Service von TecNet, um diesen stärker als bisher in ihr eigenes Dienstleistungsangebot einzubinden. 


\section{$3.9 \quad$ FAZIT}

\section{Errichtung und Organisationsstruktur}

Das TecNet wurde 1997 als ein in Europa einmaliges Modell und als eigenständige, Organisationseinheit der Innovationsagentur gegründet. Es hat seither eine Start- sowie eine Neuorientierungsphase durchlaufen. $\mathrm{Zu}$ den bedeutendsten Zielen des TecNet zählt die Verbesserung der Finanzierung von Technologieprojekten und die Unterstützung von Innovationsvorhaben durch Vermittlungsdienste und Informationsrecherchen. Der Versuch, das TecNet als reinen Vermittler zwischen Technologieexperten, Financiers und Unternehmen zu etablieren, musste mangels Nachfrage nach ca. zwei Jahren Laufzeit aufgegeben werden. Seit 1999 konzentriert sich das TecNet bei der Zielerreichung auf die Recherche von Markt- und Technologieinformationen.

Das TecNet wird lediglich von 1,5 Personen geführt, was angesichts der auferlegten Aufgaben unzureichend ist. Zum einen ist es mit dieser Personalstärke nicht möglich, ein Österreichweit wahrgenommenes und nachgefragtes Dienstleistungsangebot zu offerieren. Zum anderen muss die Bereichsleitung - neben internen Arbeitsaufgaben auch externe Kunden akquirieren, die bereit sind, die Leistungen des TecNet zu entlohnen. Neben dem Kerngeschäft der Recherche und Dokumentation werden der Bereichsleitung auch Werbung, Akquisition und die Erfüllung von Verwaltungsaufgaben abverlangt. Darüber hinaus wird eine intensivere Vernetzung und ein Schulungs-/VortragsEngagement eingefordert. Hier befindet sich das TecNet mit seinem „gewachsenen“ Angebot aber seiner nicht mitgewachsenen Organisation im Zwiespalt:

- Zum einen zählen Existenzgründer, die man auf die TecNet-Dienstleistungen aufmerksam machen will, zu seiner Zielgruppe.

- Zweitens will man junge Technologieunternehmen erreichen, denen man mit Marktinformationen bei der externen Finanzierung (Business-Pläne etc.) weiterhelfen will.

- Drittens will man Kapitalgeber und etablierte Unternehmen erreichen, denen man mit Marktinformationen Entscheidungshilfen für rentable Innovationen und neue Märkte erschließen will.

So muss sich eine Person (Bereichsleitung) auf allen Feldern gleichzeitig engagieren, was umso schwieriger ist, als dass die Aktivitäten des TecNet auf das gesamte Bundesgebiet Österreichs abzielen.

Das TecNet, das de facto aus nicht mehr als einer einzigen, als „Informationsbroker“ qualifizierten Person besteht, hat keine Vertretung, der/die bei Urlaub, im Krankheitsfall oder beim Ausscheiden der Bereichsleitung die bis dahin aufgelaufenen Projekte fertig stellen könnte. Alle Erfahrungen, Kontakte und Geschäftsabläufe konzentrieren sich auf eine Person. Scheidet diese Person aus, so löst sich im selben Moment - mehr oder weniger - auch das TecNet in seiner bis dahin bekannten Struktur auf. Es bedarf keiner Erläuterung, dass eine solche Organisationsstruktur problematisch ist. Von diesem Problem war die Evaluation unmittelbar betroffen: der persönliche Erfahrungsschatz aus 
der Startphase des TecNet 1997 bis 1999 konnte von der aktuellen Bereichsleitung nur fragmentiert wiedergegeben werden.

Verglichen mit anderen Organisationseinheiten der Innovationsagentur ist das TecNet personell nicht gewachsen und erweckt immer noch den Eindruck eines „Modells“. In Anbetracht der Zielsetzungen, der Zielgruppen, der internen Verwaltung- und externen Marketingaufgaben, des Anspruchs auf Kostendeckung und aus pragmatischen Gründen (Vertretungsregelung etc.) ist das TecNet personell unterdimensioniert.

\section{Ziele und Zielgruppen}

Bei der Begutachtung des TecNet erstaunt angesichts der unzulänglichen Personalsituation umso mehr, dass wichtige Ziele erreicht wurden. Diese Leistung ist nicht zuletzt Verdienst des großen Engagements und zahlreicher Überstunden der Bereichsleitung. Insgesamt leistet das TecNet zum zentralen Ziel der „Verbesserung und Verstärkung der Finanzierung von Technologieprojekten“ und als „Hilfsmittel zur Umsetzung von Innovationen“ einen wichtigen Beitrag. Die zahlreichen internen Anfragen der Organisationseinheit TecMa, wie auch die regelmäßigen Aufträge der BÜRGES-Förderungsbank sprechen für sich. Diese internen Aufträge sind für diese Beurteilung alleine aber nicht ausschlaggebend. Vielmehr überzeugen die externen Aufträge von Jungunternehmen an das TecNet die, wie die Fallstudien (success-stories) belegen, technologiespezifische Finanzierungsentscheidungen bedeutend geprägt haben. Hier liegt eine wesentliche Leistung des TecNet, die trotz anderer Unzulänglichkeiten nicht unterschätzt werden sollte.

Die ambitionierten Ziele, die sich die Innovationsagentur bzw. das TecNet für das Jahr 2001 im Vertrag mit dem BMWA gesetzt hat, sind nur eingeschränkt erreicht worden. Obwohl die Nettoeinnahmen des TecNet von rund 18.400 EURO im Jahr 1999 auf 22.000 EURO im Jahr 2001 angestiegen sind, sind die externen Projekteinnahmen von rund 17.000 EURO (1999/2000) im Jahr 2001 auf knapp 10.000 EURO gesunken. Hier wurde der Zielanspruch, die Nettoeinnahmen durch externe Aufträge zu erhöhen, nicht erfüllt. Gegenfinanziert wurde dieser Einnahmenausfall mit internen Projekten. Hier erhöhte sich der Betrag von rund 3.800 EURO (2000) auf ca. 12.100 EURO (2001).

In diesem Zusammenhang steht auch die Zahl der vom TecNet durchgeführten Projekte. Im Zuge der Neuausrichtung des TecNet (1999) konnte die Anzahl der durchgeführten Projekte zwar von 28 (1998) auf 41 Projekte (1999) gesteigert werden. Diese Wachstumsrate ließ aber in den folgenden zwei Jahren nach. Im Jahr 2000 und 2001 wurden jeweils 50 Projekte bearbeitet. Nach dem bemerkenswerten Anstieg der externen Projekte mit Leistungsverrechung zwischen 1998 (3 Projekte) und dem Jahr 2000 (29 Projekte), wurde das Ziel für das Jahr 2001, die Zahl der durchgeführten Projekte insbesondere für externe Auftraggeber zu erhöhen mit nur 17 realisierten Projekten verfehlt. Dieses Verhältnis bleibt auch im Saldo negativ, wenn man die externen Projekte ohne Leistungsverrechung (Marketingmaßnahmen etc.) hinzuzählt.

Für eine ausgeglichene Betrachtung sind diese Ergebnisse jedoch in den wirtschaftlichen und politischen Gesamtzusammenhang zu stellen: Nach einer Euphorie an den Technologiemärkten im Jahr 2000 und in Erwartung der Fortführung dieses Trends (Zielsetzung 
des TecNet) führte eine allgemeine Konjunkturschwäche in 2001 dazu, dass die Ziele zu hoch gesteckt worden waren. Darüber hinaus hatte die Innovationsagentur zu diesem Zeitpunkt Unwägbarkeiten bei der weiteren Finanzierung des TecNet in Kauf zu nehmen. Da die Vertrags- und Finanzierungssituation lange Zeit ungeklärt war, konnte auch das TecNet seine Aktivitäten nicht mit dem erforderlichen Rückhalt weiterverfolgen.

Die jährlichen Zielvereinbarungen der Bereichsleitung von TecNet mit der Geschäftsführung der Innovationsagentur (interne Ziele) wurden dagegen punktgenau erreicht. Ziel war es, im Zweijahreszeitraum 2000/2001 insgesamt 100 Projekte sowie ca. 80 Anfragen zu bearbeiten. TecNet hat dieses Ziel, trotz des schwächer notierten Technologiemarktes, erreicht. Verwunderlich ist allerdings, dass genau 50 Projekte im Jahr 2000 und 50 Projekte im Jahr 2001 bearbeitet wurden. Da nicht anzunehmen ist, dass TecNet weitere Projekte nach der Zielerreichung abgelehnt hätte, muss davon ausgegangen werden, dass große Anstrengungen unternommen wurden, genau 50 Projekte zu bearbeiten. Der hohe Anteil interner Projekte in 2001 birgt allerdings auch die Gefahr, dass das ,50 ProjekteZiel" nach Absprache/Abstimmung mit anderen Organisationseinheiten der Innovationsagentur erfolgte. In diesem Fall müsste man von einer unerwünschten Quersubventionierung ausgehen. Da die anderen Organisationseinheiten jedoch nicht Gegenstand dieser Evaluation waren, liegen dem Evaluationsteam keine Hinweise auf Quersubventionierung vor.

Die ursprüngliche Zielgruppe der Geld- und Kapitalgeber (1997) wurde in der Phase der Neuausrichtung des TecNet (1999) um junge High-Tech-Unternehmen, Erfinder, Universitätsinstitute, KMUs und andere Industriebetriebe erweitert. Darüber hinaus wurde im Jahr 2001 der Ziel- und Aufgabenkatalogs des TecNet auf Marketingaktivitäten ausgedehnt, die das TecNet, neben seiner Hauptaufgabe der Markt- und Technologierecherche, zur Erstellung von Informationsmaterialien und Vorträgen verpflichtet. Diese Ausweitung des Ziel- und Arbeitsprogramms von Seiten der Innovationsagentur bzw. des Auftraggebers (BMWA) ist ohne eine personelle Verstärkung ist nicht nachvollziehbar.

Die Vielzahl der Adressaten erscheinen dem Evaluationsteam als ungerechtfertigt. Aus wirtschaftspolitischer Sicht, ist eine Förderung allenfalls für Erfinder, Unternehmensgründer und junger Technologieunternehmen legitim. Es wäre zwar wünschenswert, wenn etablierte bzw. Großunternehmen durch ertragsstarke Projekte die kapitalschwachen Unternehmensgründer ausgleichend subventionieren, aber nur dann, wenn das TecNet über eine entsprechende Organisation verfügt und unterschiedliche Preise diese Konstruktion stützen. Ansonsten ist zu befürchten, das TecNet - auch aufgrund der Popularität und des Erfolgs großer Unternehmen wegen - einzelne Großprojekte bevorzugt anstatt sich der bedürftigen Zielgruppe zu widmen. Im Tagesgeschäft des TecNet ist zu vermuten, dass diese Preisdifferenzierung indirekt durch die Abrechnung einer unterschiedlichen Zahl Arbeitsstunden praktiziert wird. Ein Großunternehmen zahlt eher für den realen Arbeits- und Rechercheaufwand, als ein KMU, bei dem der Arbeitsaufwand in die kostenlose Vorrecherche verlagert wird (s. Finanzierung). Diese Form der ausgleichenden Verrechnung ist zwar lobenswert, beinhaltet aber aufgrund ihrer Beliebigkeit auch zahlreiche Schwachpunkte. 
Darüber hinaus will das TecNet zwar Klienten (potenzielle Gründer) im Hochschulsektor ansprechen, es sollte jedoch nicht zu seinen Aufgaben zählen hier Vorlesungen zu Rechercheverfahren anzubieten. Ein Image-Folder, einzelne Präsentationen auf Gründertagen wäre angemessen, ein mehrtägiges Seminar oder ein Recherche-Workshop angesichts der knappen Kapazitäten aber verfehlt. Die angepeilte Kooperation mit den AplusB-Zentren (vorbehaltlich der konkreten Ausgestaltung) scheint allerdings ein Weg in die richtige Richtung zu sein.

\section{Wettbewerbsumfeld}

Die Preis-/Leistungsrelation bei EDV-Geräten und die neuen Möglichkeiten der Kommunikationsmedien versetzen sowohl Unternehmensgründer als auch kleine und mittlere Unternehmen zunehmend besser in die Lage auf elektronische Informationen zuzugreifen. Datenbankanbieter, Marktforschungsinstitute, Wirtschaftsauskunfteien, Universitätseinrichtungen etc. bieten ihre Produkte und Leistungen in steigendem Umfang im Internet an. Für die Dienstleistungen des TecNet ist diese Entwicklung marktentscheidend, da die Zielgruppe technisch grundsätzlich in der Lage ist Informationen eigenständig zu recherchieren. Gleiches gilt für Drucksachen, wie z.B. Markt- und Branchenberichte die sich über Verbände oder öffentliche Transfereinrichtungen beschaffen lassen, zunehmend sogar als geschützte PDF-Dateien über das Internet.

Trotz dieser Entwicklung ist das Marktpotenzial von TecNet als gut einzuschätzen. TecNet agiert in einem Wachstumsmarkt und bietet wesentliche Vorteile, die am Markt für Informationsdienstleistungen einen Preis ermöglichen: (a) Rechercheerfahrung im Umgang mit Datenbanksystemen, (b) die fachlich-kompetente Bewertung der Informationsqualität, (c) die daraus resultierenden Zeitersparnisse sowie (d) Kostenersparnisse bei langfristigen Datenbankverträgen bzw. durch Auszüge kostenpflichtiger Marktstudien.

\section{Marketing}

Bei den Zielsetzungen im Bereich Marketing wurden die Anforderungen weitgehend erfüllt. So ist z.B. die Drucklegung eines professionellen Image-Folders in Vorbereitung. Bis heute hat sich das TecNet mit selbsterstellten Marketingunterlagen, einer Homepage, „Success-Stories“ und zahlreichen Vorträgen auf Fachveranstaltungen seinen Kunden präsentiert. In einigen Fällen wurden auch kostenlose Recherchen durchgeführt, um strategisch wichtige Kundenkreise mit den Dienstleistungen des TecNet vertraut zu machen. Als sehr ansprechend präsentiert sich das TecNet im Außenbild. Das Corporate Design (Logo TecNet, Innovationsagentur, Farbgebung, Aufbau etc.) als Bestandteil der Marketingaktivitäten ist sehr gelungen, wobei einzig die lange Namensgebung „TecNet Das Netzwerk für Markt- und Technologieinformationen“ ggf. falsche Assoziationen bei den Leistungen hervorruft. Die Unterlagen für Präsentationen sind vorbildlich aufbereitet und vermitteln die hohe Kompetenz im Bereich der Informationsvermittlung - was letztlich auch auf Neukunden ausstrahlt.

Kritisch ist allenfalls zu bemerken, das sich die Gestaltung und Drucklegung des vierseitigen Image-Folders seit dem Jahr 2001 hinzieht (Jahresbericht 2001 nennt Februar 2002 als Drucktermin, inzwischen wurde Juni 2002 genannt). Im zukünftigen Image- 
Folder werden ganz allgemein Unternehmen, Kreditgeber und Investoren angesprochen. Das TecNet wendet sich dieser Zielgruppe, gemäß seiner vertraglichen Pflichten von 2001, zu. Jedoch wird gerade die Zielgruppe der Start-Up-Unternehmen und KMU in allen Verträgen und Äußerungen als besonders relevant bezeichnet. Ein Unternehmensgründer oder Erfinder, vor allem aber „Business Angels“ als Kapitalgeber finden sich in den Werbefoldern nur schwer wieder. In den Success-Stories des TecNet werden vielmehr die Erfahrungen etablierter Unternehmen beschrieben. Wie bereits angemerkt wurde, steht die Frage im Raum, ob ein derart breit adressiertes Kundensegment - bei gegebener Größe des TecNet - zweckdienlich ist.

Auch hinsichtlich der Vortragsveranstaltungen und Pressearbeit wird einer allgemeinen Präsenz gegenüber einer gezielten Kundenansprache der Vorzug gegeben. Es entsteht zudem der Eindruck, dass TecNet auf einigen Veranstaltungen weniger mit seinem Angebot um Kunden wirbt, als vielmehr Weiterbildungsseminare zum eigenständigen Recherchieren von Marktinformationen anbietet. Dieser Eindruck verstärkt sich durch die neue Rubrik „Vorlesungen/Schulungen“ im Jahresbericht 2001 (S. 2). Auch sind Präsentationen vor Mitarbeitern der BÜRGES Förderungsbank und neuer Mitarbeiter der Innovationsagentur eher als interne Informationsveranstaltungen denn als „Öffentlichkeitsarbeit" anzusehen.

Eine Konzeption für eine ,gezielte“ PR- und Medienarbeit ist schwer auszumachen. Dies ist aber nicht dem TecNet, sondern ganz allgemein der Innovationsagentur anzulasten: Einerseits werden dem TecNet vertraglich zahlreiche Marketingaktivitäten auferlegt, andererseits wird von der Innovationsagentur ein eigener Organisationsbereich „Marketing“ aufgebaut. Trotz dieser Befunde hat das TecNet das im Vertrag vom 30. Mai 2001 definierte Arbeitsprogramm (Marketing) erfüllt bzw. es ist absehbar, dass die Anforderungen (Image-Folder) vor Vertragsablauf erfüllt werden. Die o.g. Kritik richtet sich somit nicht an die Arbeitsweise des TecNet, sondern an die Gesamtstrategie der Innovationsagentur und stellt die vertraglichen Anforderungen (Marketing) als zusätzlichen Aufgabenbestandteil an das TecNet in Frage.

Ganz allgemein betonen die Marketing-Aktivitäten des TecNet Effizienzvorteile (Zeitersparnisse, Kostenersparnisse etc.), nicht jedoch den bedeutenden Vorteil Markt- und Technologieinformationen zur Absicherung von Finanzierungsentscheidungen bzw. strategischen Entscheidungen „unabhängig“ zu recherchieren. Hier bietet sich für das TecNet jedoch ein entscheidendes Argument, das bislang kaum kommuniziert wird. Denkbar wäre in diesem Zusammenhang, das ein kapitalsuchendes Unternehmen (HighTech Start-Up, Erfinder etc.) die TecNet-Dienstleistung nicht mehr nur in eigenen Berichten/Anforderungen verwertet, sondern direkt den Kapitalgebern (Banken, VC, Business-Angels) als „unverfälschte“ Informationsgrundlage zur Verfügung stellt. In diesem Segment „unabhängige Recherche und Informationsermittlung“ ist Potenzial zu vermuten, womit sich das TecNet nicht nur als bezahlter Informationsvermittler sondern auch als objektiver Informationsanbieter positionieren kann. 


\section{Auftragsabwicklung}

In der Anfangszeit des TecNet war mit hohen „Startkosten“ (Erfahrungsaufbau) zu rechnen. Die Umorientierung des TecNet, von der Expertenvermittlung hin zur Recherche technologierelevanter Marktinformationen war ein notwendiger Schritt, der aber auch dazu führte, dass eine neue Arbeitsorganisation erforderlich wurde. Neben diesem inhaltlich-aufgabenbezogenen Wandel, führte ein personeller Wechsel in der Bereichsleitung auch zu einem Neuanfang in der Projektakquisition und -bearbeitung. Da die Organisationseinheit TecNet über keine vergleichbar kompetenten Stellvertreter in der Bereichsleitung verfügt, wurde das TecNet in 1999 mit neuen Inhalten und Personen quasi neu gegründet. Die Messlatte der Lerneffekte und Effizienz, die man an vergleichbare Organisationen nach vier Jahren ihrer Tätigkeit richtet, reduziert sich aufgrund der Neustrukturierung des TecNet somit auf die Jahre 2000 und 2001. Innerhalb dieser zwei Jahre wurden folgende produktivitätssteigernde Prozesse in die Arbeit implementiert, die belegen, das TecNet seine Ablauforganisation kontinuierlich verbessert:

- Einführung einer exakten Projektdokumentation (Kosten- und Leistungsrechnung, Kundenservice, juristische Absicherung etc.)

- Erstellung von Projektangeboten (Vorrecherche, Machbarkeitsprüfung)

- Erstellung von Projektendberichten (Produktwahrnehmung, Leistungsnachweis)

- Kostenersatz (Leistungs- und Qualitätsbewusstsein)

Der Arbeitsablauf ist insofern weiter verbesserungswürdig, als dass eine Tendenz zu beobachten ist, dass bei der Auftragsanbahnung Informationen geliefert werden, die der potenzielle Kunde für eigene Recherchen nutzt - ohne die Dienste des TecNet zu beanspruchen. Beispielweise führt die Vorrecherche zu dem Ergebnis, dass es bestimmte Studien bzw. Datenbanken oder Internet-Links gibt, die mit dem potenziellen Kunden auf ihre Relevanz für das eigentliche Projekt diskutiert werden. Der Kunde hat auf diese Weise bereits die für ihn wertvollen Informationsquellen erfahren, ohne dafür zu zahlen. Hier besteht die Gefahr, dass das TecNet, wie bereits in den ersten beiden Jahren seines Bestehens, „umlaufen“ wird. Die Tatsache, dass im Jahr 2001 rund die Hälfte aller Projektanfragen zu keinem Auftrag führten, sowie der lange organisatorische Vorlauf (Vorgespräch, Vorrecherche, Projektangebot) legen ein solches Verhalten, trotz des fairen Preisangebots nahe. Es erfordert von Seiten der Projektleitung große Erfahrung, dem Kunden nur so viel (wertvolle) Information wie nötig, aber bezüglich der Auftragserteilung, so viele Anreize wie möglich zu geben. Hier gibt TecNet selbstkritisch an, dass „phasenweise zu viele Ergebnisse/Informationen bei der Angebotserstellung“ preisgegeben werden.

Im Hinblick auf die Auftragslage entfallen jährlich inzwischen mehr als die Hälfte aller Projekte auf die interne Leistungsverrechnung. Dies ist insofern erstaunlich, als dass das Ziel der Verstärkung von Marketingaktivitäten (2000 und 2001) keinen Einfluss auf die externe Projektakquisition zu haben scheint. Hier liegt der Schluss nahe, dass internen Projekten der Vorzug gegeben wird. Eine solche Präferenz wäre aufgrund des geringeren 
Aufwands und angesichts der Personalsituation nachvollziehbar. An dieser wie an anderer Stelle ist offensichtlich, dass bei externen Aufträgen sowohl ein Anreiz- als auch ein Kapazitätsproblem auftritt. Für TecNet ist es derzeit sicherlich weniger aufwendig, interne Projekte zu bearbeiten (,kurze Wege“, Abstimmungsvorteile etc.) als sich vergleichsweise aufwendigen externen Projekten zuzuwenden.

\section{Finanzierung}

In der Projektabwicklung zeichnet sich im beschriebenen Vorlauf bis zum Vertragsabschluss ein Schlüsselproblem ab: Zunächst ist die Projektleitung des TecNet in der Akquisitionsphase arbeitstechnisch stark gefordert und bemüht dem anfragenden Kunden in kürzester Zeit bestmögliche Ergebnisse (Recherchemöglichkeiten) zu präsentieren. Gleichzeitig ist die Vorrecherche auch für das TecNet von hoher Bedeutung (Renommee), um sich nach Auftragserteilung nicht mit minderwertigen Informationen offenbaren zu müssen. Das bedeutet, dass ein Großteil der eigentlichen Arbeit im Vorfeld eines Vertrags erbracht wird - ohne dass diese Anbahnungskosten z.B. durch andere Projekte gedeckt sind. Bei einer bereitwilligen Präsentation der Ergebnisse der Vorrecherche läuft das TecNet anschließend Gefahr, den Kunden durch eine unfreiwillige Informationspreisgabe zu verlieren. In diesem Fall wurden zwar Lerneffekte erzeugt, der Arbeitsaufwand musste jedoch ohne Ertrag abgeschrieben werden. Kommt es dennoch zu einem externen Auftragsverhältnis, so treten weitere Probleme auf, die den Kostendeckungsgrad negativ beeinflussen: Zum einen wird den Kunden nach Auftragersteilung erst die von diesem Moment anfallenden Kosten (Stundensätze) abverlangt, wobei die Grundpauschale nicht den Vorrechercheaufwand decken kann. Zum anderen verfügt ein Teil des Zielklientels (Unternehmensgründer, jungen High-Tech-Firmen) nur über ein sehr begrenztes Budget, so dass ein finanziell attraktives Projekt sich bei diesen Firmen kaum realisieren lässt. Bei der Verrechnung interner Projekte entfällt die Grundpauschale vollständig. Das Evaluationsteam kann zudem nicht bemessen, inwiefern die verrechneten Stunden dem tatsächlichen Arbeitsaufwand entsprechen - oder ob im täglichen Geschäft eher ,pauschal“ für eine bestimmte Leistungserbringung abgerechnet wird.

Ein Beispiel soll den hohen Anteil der Arbeitskosten verdeutlichen, denen keine Erträge gegenüberstehen: Bemisst man für die Kostendeckung, das einige Recherchen 30 bis 80 Stunden Arbeitsaufwand bedeuten ${ }^{3}$, so müssten diese Projekte (intern, auch ohne Grundpauschale) mit mindesten $€ 2.725,00(30 \mathrm{x} € 90,84)$ bzw. $€ 7.267,20(80 \mathrm{x} € 90,84)$ abgerechnet werden. Im Jahr 2001 wurden das größte Projekte mit $€ 2.725,23$ abgerechnet, der Durchschnitt aller Projekte liegt bei $€ 412,00$ (extern) bzw. $€ 466,00$ (intern), so dass gemessen an den Arbeits- und Überstunden pro Jahr hohe Kosten im Vorfeld der honorierten Recherchen und für andere Aufgabenbereiche anfallen.

\footnotetext{
${ }^{3}$ vgl. Jahresbericht 2001, Punkt 3
} 


\section{Potenzialanalyse und Unternehmensbefragung}

In diesem Kapitel werden die Aktivitäten des TecNet in Relation zum Gründungsgeschehen technologieorientierter Unternehmen in Österreich gestellt. Da es sich bei dieser Zielgruppe um eines der wichtigsten Kundensegmente des TecNet handelt, wird abgeschätzt, welche Marktbedingungen für Informationsbroker in Österreich vorherrschen. Eine schriftliche Befragung gibt Aufschluss über die Zufriedenheit bisheriger TecNet-Kunden, das Marktpotenzial der angebotenen Dienste bei potenziellen Neukunden sowie die Bekanntheit des TecNet in der Wissenschaft und bei Förderinstitutionen.

\subsection{POTENZIALANALYSE}

\subsubsection{Gründungsdaten Österreich}

Die Berichterstattung zum Unternehmensgründungsgeschehen in Österreich erfolgt im Auftrag des Bundesministeriums für Verkehr, Innovation und Technologie (BMVIT). Die österreichischen Gründungsdaten werden durch das Zentrum für Europäische Wirtschaftsforschung (ZEW) und die JOANNEUM RESEARCH Forschungsgesellschaft $\mathrm{mbH}$ ausgewertet.

In Deutschland wurde im Jahr 1991 am ZEW in Kooperation mit der größten Handelsauskunftei CREDITREFORM zunächst mit dem Aufbau umfangreicher Paneldateien zu deutschen Unternehmen begonnen. Für die Analyse des Unternehmensgründungsgeschehens in Österreich wurde die Zusammenarbeit mit CREDITREFORM auf Österreich ausgedehnt. CREDITREFORM transferiert seine Unternehmensrecherchen im Halbjahresabstand vollständig an das ZEW, so dass zum gegenwärtigen Zeitpunkt Informationen zu ca. 305.000 österreichischen Unternehmen zur Verfügung stehen. Mit den einzelnen Datenwellen, die jeweils den gesamten Unternehmensbestand umfassen, gelangen nicht nur neu erhobene Unternehmen in das Gründungspanel Österreich, sondern es werden auch zwischenzeitlich aktualisierte Angaben zu bereits im Datenbestand enthaltenen Unternehmen übernommen.

Die Datenerhebung seitens CREDITREFORM erfolgt dezentral, jedoch nach einem einheitlichen Modus, durch insgesamt acht eigenständige Niederlassungen. ${ }^{4}$ CREDITREFORM führt eine systematische Recherche verschiedener öffentlicher Register (insbesondere des Handelsregisters), der Konkurs- und Vergleichsmeldungen sowie von Tageszeitungen, Geschäftsberichten und veröffentlichten Bilanzen durch. Neben dem Handelsregister stellen die durch Kundenanfragen ausgelösten Recherchen die wichtigste Quelle für die Erfassung von Unternehmensinformationen dar.

\footnotetext{
4 Dabei handelt es sich um Wien, Salzburg, Linz, Villach, Graz, Innsbruck, Bregenz und St. Pölten.
} 
Diese Daten werden im Rahmen der Evaluierung als Basis für die Potenzialanalyse verwendet.

\subsubsection{Analyse des Kundenpotenzials}

Gemäß der von TecNet adressierten Zielgruppe von High-Tech Start-Ups wird untersucht, wie sich die Unternehmensgründungen in technologieorientierten und wissensintensiven Branchen zwischen 1997 und 2000 in Österreich entwickelt haben. ${ }^{5}$ Die Sektorstruktur gibt Anhaltspunkte darauf, welches Kundenpotenzial TecNet gegenüberstand bzw. welche Bedeutung diese Klientel für TecNet beizumessen ist. Die Abgrenzung der hier interessierenden Branchen (FuE-intensive Industrie- und Dienstleistungsbranchen, wissensintensive Dienstleister bzw. nicht-technische Berater) erfolgt nach der branchendurchschnittlichen FuE-Intensität (Anteil der Aufwendungen für FuE am Umsatz).

Zwischen 1997 und 2000 lässt sich für Österreich ein Anstieg des Anteils FuE-intensiver Gründungen feststellen, der im wesentlichen von der Dynamik im Dienstleistungssektor geprägt wird. Während der Anteil der FuE-intensiven Gründungen im verarbeitenden Gewerbe bei rund 1,6\% an allen Gründungen stagniert, steigt der Anteil der FuEintensiven Dienstleistungsgründungen um drei Prozentpunkte (von sechs auf neun Prozent an). Im Vergleich zu Westdeutschland ist der Anteil Österreichs in diesen beiden Segmente mittlerweile sogar höher, so dass die Marktbedingungen für TecNet in Bezug auf den Kundenkreis ,junge Technologieunternehmen“ besonders günstig waren.

Berücksichtigt man den regionalen Wirkungskreis von TecNet, der sich überwiegend auf Wien und Niederösterreich konzentriert, so hat sich das Gründungsgeschehen anhaltend dynamisch entwickelt: Sowohl im verarbeitenden Gewerbe als auch im Dienstleistungssektor sind deutliche Unternehmenszuwächse durch High-Tech Start-Ups festzustellen (vgl. Tabelle 6).

Tabelle 6: $\quad$ Anteil technologieorientierter Gründungen an allen Gründungen in \%

\begin{tabular}{lcccccc}
\hline \multicolumn{1}{c}{$\mathbf{1 9 9 0}$} & $\mathbf{1 9 9 1 / 9 2}$ & $\mathbf{1 9 9 3 / 9 4}$ & $\mathbf{1 9 9 5 / 9 6}$ & $\mathbf{1 9 9 7 / 9 8}$ & $\mathbf{1 9 9 9 / 0 0}$ \\
FuE-intensive Branchen im Verarbeitenden & Gewerbe & & & & \\
\hline Österreich & 1,41 & 1,71 & 1,66 & 1,50 & 1,69 & 1,55 \\
Wien & 0,97 & 1,55 & 1,02 & 1,18 & 1,34 & 1,59 \\
Kernstädte & 1,65 & 1,64 & 2,21 & 1,45 & 1,83 & 1,34 \\
Stadtumland & 1,73 & 1,69 & 1,64 & 2,09 & 2,15 & 1,58 \\
Sonstige Bezirke & 1,48 & 1,92 & 2,00 & 1,45 & 1,86 & 1,71 \\
\hline FuE-intensive Dienstleistungssektoren & & & & & \\
\hline Österreich & 6,08 & 6,42 & 6,68 & 7,53 & 7,71 & 9,00 \\
Wien & 6,57 & 6,47 & 7,26 & 9,07 & 8,82 & 11,73 \\
Kernstädte & 7,93 & 8,03 & 7,72 & 8,35 & 8,79 & 9,33 \\
Stadtumland & 6,39 & 6,96 & 7,45 & 7,43 & 7,40 & 8,25 \\
Sonstige Bezirke & 4,00 & 4,90 & 5,00 & 5,27 & 5,98 & 7,03 \\
\hline
\end{tabular}

5 Die aktuelle Statistik zum Gründungsgeschehen in Österreich endet im Jahr 2000. 
Betrachtet man dieses Bild sektorspezifisch, d.h. Anzahl der technologieorientierten Gründungen im verarbeitenden Gewerbe an allen Gründungen im verarbeitenden Gewerbe, resp. Anzahl der technologieorientierten Gründungen im Dienstleistungssektor an allen Gründungen im Dienstleistungssektor, so zeigt sich: Ende der 90er Jahre sind in Österreich etwa $17 \%$ aller Gründungen im verarbeitenden Gewerbe FuE-intensiven Wirtschaftszweigen zuzuordnen (gegenüber ca. $32 \%$ in Westdeutschland). Im Bezug auf den Bereich der unternehmensnahen Dienstleistungen ist jedoch ein gegenteiliges Bild festzustellen. Der Anteil forschungs- und wissensintensiver Dienstleistungsgründungen ist in Österreich fast durchwegs höher als in deutschen Vergleichsregionen.

Im Großraum Wien hat sich der Anteil zudem von 44 \% (1997/1998) auf knapp $47 \%$ (1999/2000) gesteigert. Erste Prognosen für das Jahr 2001 gehen allerdings davon aus, das sich dieser positive Trend abgeschwächt hat. Diese Beobachtungen decken sich weitgehend mit der Anzahl der von TecNet bearbeiteten Projekten. Für den Zeitraum 1998 bis 2000 ist ein kontinuierlicher Zuwachs von insgesamt 28 auf 50 Projekte festzustellen. Insbesondere die Zunahme der externen Aufträge steht im Einklang mit der positiven Gesamtentwicklung.

In absoluten Zahlen liegt die Anzahl der technologieintensiven Neugründungen in Österreich bei rund 1.500 Firmen pro Jahr. ${ }^{6}$ Es wird geschätzt, dass etwa 500 dieser Neugründungen ihren Unternehmenssitz in Wien und Niederösterreich haben. Knapp 85 $\%$ dieser Gründungen sind den technologieintensiven Dienstleistungen (Fernmeldedienste, Datenverarbeitung und Datenbanken, Forschung und Entwicklung etc.) zuzurechnen. $15 \%$ der Firmen sind Gründungen in den Wirtschaftszweigen des verarbeitenden Gewerbes.

Aufgrund dieser Größenordnung lässt sich abschätzen, dass das TecNet im Großraum Wien/Niederösterreich mit anderen Anbietern um rund 500 High-Tech Start-Ups pro Jahr konkurriert. Der weitaus größte Teil dieser Firmen sind Dienstleistungsgesellschaften. Es bleibt jedoch $\mathrm{zu}$ beachten, dass es sich hierbei auch um Firmen handelt, die als selbständige Töchter von großen Telekommunikationsanbietern, Beratungsgesellschaften etc. gegründet werden. Nicht jede dieser technologieorientierten Unternehmensgründungen fällt damit in die Zielgruppe des TecNet. ${ }^{7}$

\subsection{UNTERNEHMENSBEFRAGUNG}

\subsubsection{Datenerhebung}

Im Rahmen dieser Evaluation wurden insgesamt 44 ehemalige Kunden des TecNet sowie 175 Unternehmen und Wissenschaftseinrichtungen kontaktiert. Die Auswahl der 175 potenziellen TecNet-Kunden erfolgte durch eine geschichtete Zufallsstichprobe (Branche,

\footnotetext{
${ }^{6}$ Durchschnittswerte der Jahre 1990 bis 2000. Unternehmen der Spitzentechnik, der höherwertigen Technik und technologieintensive Dienstleister.

${ }^{7}$ Eine genauere Spezifizierung ist im Rahmen dieser Studie nicht möglich.
} 
Unternehmensgröße, Alter, Region). Alle Unternehmen und Einrichtungen wurden anschließend mit einem Begleitbrief des BMWA sowie einem Fax-Fragebogen von JOANNEUM RESEARCH angeschrieben (Materialien 6-11). Der Fax-Fragebogen hatte in Abhängigkeit vom Statuts des angeschriebenen Unternehmen (TecNet-Kunde/kein TecNet-Kunde) unterschiedliche Fragenblöcke (Materialien 7 und 9).

Bei den Nicht-Kunden des TecNet handelt es sich um eine Zufallsauswahl technologieund wissensintensiver Firmen in ganz Österreich. Die Adressen wurden aus einer aktuellen Marketingdatenbank (HEROLD Marketing) und aus der Gründungsdatenbank Österreich des Zentrums für Europäische Wirtschaftsforschung (ZEW) exportiert. Darüber hinaus wurden zehn Schlüsselpersonen der Forschungsförderlandschaft Österreichs mit einem separaten Fax-Fragebogen angeschrieben und um Informationen sowie eine Stellungnahme gebeten (vgl. Materialien 10 und 11).

Die schriftliche Befragung erfolgte im Zeitraum vom 10. bis zum 23. Juni 2002. In einer Erinnerungsaktion wurden anschließend alle Unternehmen antelefoniert, von denen man bis zum 23. Juni 2002 noch keine Antwort erhalten hatte. Diese Unternehmen wurden nochmals um die Beantwortung der Fax-Fragebögen gebeten. In vielen Fällen wurde der Fragebogen dem Unternehmen wiederholt zugesandt. Die Umfrage wurde am 10. Juli 2002 abgeschlossen.

Allgemein ist festzustellen, dass viele Unternehmen die Thematik mit großem Interesse aufgenommen haben: Von den 175 angeschriebenen Firmen, die bislang noch keine TecNet-Kunden sind, haben $37 \%$ den Fragebogen beantwortet. Bei den 44 kontaktierten TecNet-Kunden lag die Antwortrate bei $36 \%$. Es wurden nicht alle TecNet-Kunden angeschrieben, da einige der High-tech Firmen bereits wieder vom Markt verschwunden sind, oder der ehemals zuständige Ansprechpartner die Firma verlassen hat.

Tabelle 7: $\quad$ Angeschriebene und antwortende Unternehmen

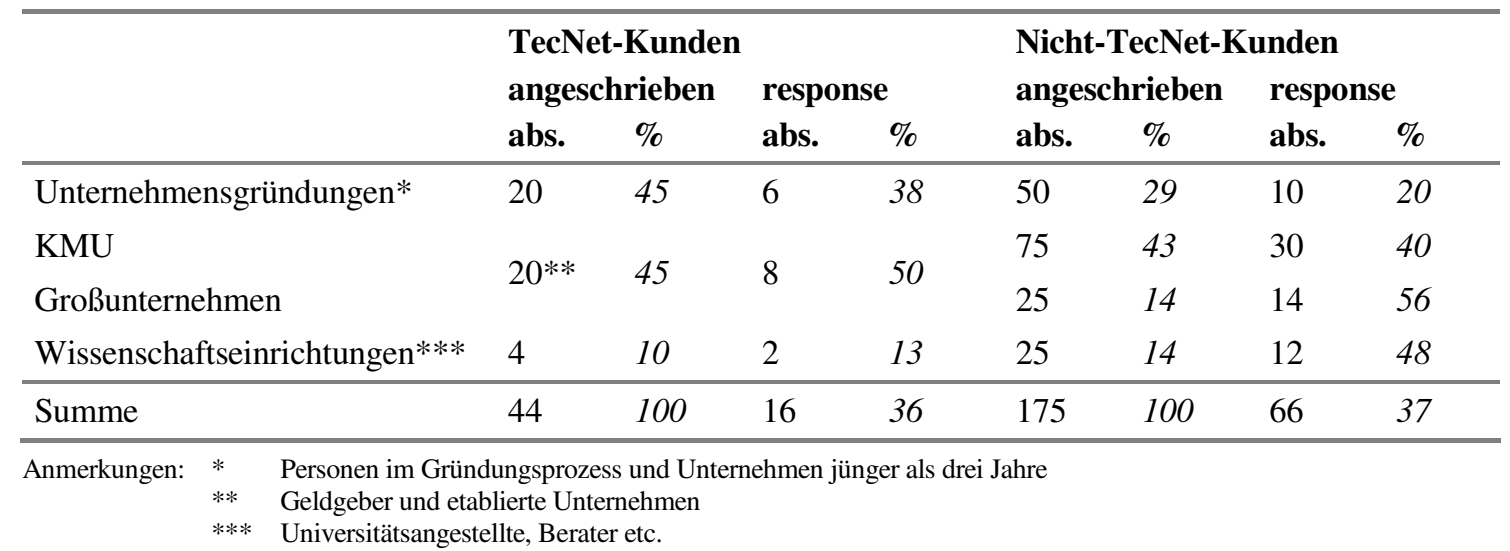

Darüber hinaus konnte nicht jede aus den Unternehmensdatenbanken selektierte Firma mit der schriftlichen und telefonischen Befragung erreicht werden. Bei fünf Unternehmen (ca. $3 \%$ ) konnte der Fragebogen/Brief nicht zugestellt werden, weil das Unternehmen an der angegebenen Adresse nicht aufzufinden war. Ein Unternehmen verweigerte die Auskunft aufgrund eines laufenden Konkursverfahrens. In der telefonischen Erinnerungsaktion zeigten 14 Unternehmen (8\%) kein Interesse und bei 16 Firmen (9\%) 
meldete sich trotz eines zweimaligen Anrufversuchs kein Gesprächspartner. In den übrigen Fällen war man unentschlossen und hat die Rückantwort z.B. von der verfügbaren Zeit abhängig gemacht.

\subsubsection{Ergebnisse der Unternehmensbefragung}

\section{Beurteilung des TecNet durch seine Mandanten}

Von den 44 an TecNet-Kunden entsandten Fragebögen wurden 16 zurückgesendet. Bei diesen Fax-Antworten wurden zwei Fragebögen von unterschiedlichen Abteilungen/ Sachbearbeitern derselben Firma (BÜRGES-Förderungsbank und der Mittelstandsfinanzierungs AG) zurückgesandt. Insgesamt wurden somit 14 verschiedene TecNetKunden befragt.

Nahezu $90 \%$ aller antwortenden Kunden haben weniger als 250 Mitarbeiter und zählen somit zu den kleinen und mittleren Unternehmen in der Volkswirtschaft Österreichs; zehn Mandanten (63\% der antwortenden Kunden) beschäftigen sogar weniger als 50 Mitarbeiter. Trotz ihrer geringen Größe sind immerhin neun Kunden des TecNet (56 \%) als Tochtergesellschaften in einen Konzern oder in ein Großunternehmen eingebunden. Diese Verflechtungen korrespondieren auch mit dem Grad der Internationalisierung. Lediglich vier Unternehmen $(25 \%)$ sind ausschließlich in Österreich mit ihren Produkten vertreten. Alle anderen Firmen bieten ihre Produkte europaweit oder sogar weltweit an.

Das TecNet ist den meisten Kunden (75\%) über andere Einrichtungen der Innovationsagentur bekannt geworden. Es ist anzunehmen, dass vor allem die Bereiche TecMa und SeedCapital auf die Dienste des TecNet hingewiesen haben und es im Zuge dieser Kontakte zu einem Auftragsverhältnis gekommen ist (vgl. Materialien 25, Expertengespräche [2]). Drei Kunden sind über eigene Recherchen auf das TecNet aufmerksam geworden und drei weiteren Kunden wurde das TecNet empfohlen. Es fällt auf, das kein Unternehmen durch Presseartikel, Fachzeitschriften oder einen Werbefolder die Leistungen des TecNet kennengelernt hat (vgl. Tabelle 8).

Tabelle 8: $\quad$ Bekanntheit des TecNet

\begin{tabular}{lll}
\hline Das TecNet ist Ihnen bekannt durch... & abs. & in \% \\
\hline andere Einrichtungen der „Innovationsagentur“ & 12 & 75 \\
Messen/Tagungen/Fachveranstaltungen etc. & 1 & 6 \\
eigene Recherchen (z.B. TecNet-Homepage) & 3 & 19 \\
Empfehlung/Referenzen & 3 & 19 \\
Presse/Fachmagazine/Werbefolder & 0 & 0 \\
Ihre Bank/Venture Capital Gesellschaft & 1 & 6 \\
Berater (Gründungsberater, Consultants etc.) & 1 & 6 \\
\hline
\end{tabular}

Mehrfachantworten möglich

Die Erwartungen, mit denen sich die befragten Firmen an das TecNet wenden, liegen in Informationsbausteinen für unternehmensstrategische Entscheidungen. Vielfach wird die Bereitstellung einer vollständigen Marktrecherche erwartet. Jeweils fünf Kunden geben 
an, sie sich vom TecNet die Recherche von Technologieinformationen bzw. den Zugang zu unbekannten Informationsquellen erhofften (vgl. Tabelle 9).

Tabelle 9: $\quad$ Erwartungshaltung an das TecNet

\begin{tabular}{lll}
\hline Ihre Erwartungshaltung an TecNet war... & abs. & in \% \\
\hline spezifische Informationsbausteine zu erhalten & 10 & 63 \\
die Bereitstellung einer kompletten Marktrecherche & 10 & 63 \\
die Recherche nach Technologieinformationen & 5 & 31 \\
Kontakte zu Experten (Uni, FH etc.) herzustellen & 1 & 6 \\
die Erfüllung von Anforderungen „Dritter“ & 1 & 6 \\
der Zugang zu bislang unbekannten Infoquellen & 5 & 31 \\
\hline
\end{tabular}

Mehrfachantworten möglich

Die Dienstleistungen des TecNet umfassen vier Rubriken: die Bereitstellung (a) von Marktinformationen, (b) von Technologieinformationen, (c) die Vermittlung von Experten, sowie (d) die direkte Beratung durch TecNet-Mitarbeiter. In der Umfrage wurde ermittelt, mit welchen Anteilen diese einzelnen Leistungen in das gesamte Dienstleistungspaket des TecNet an einen Kunden eingehen. Insgesamt sechs Unternehmen geben an, ausschließlich Marktinformationen erhalten haben. Weitere acht Firmen schätzen den Anteil der Marktinformationen auf immerhin noch $50 \%$ bis $80 \%$ der insgesamt erbrachten Dienstleistung. Die verbleibenden Prozente verteilen sich auf die Bereitstellung von Technologieinformationen (Mittelwert $32 \%$ ) sowie auf die unmittelbare Beratung durch das TecNet selbst (Mittelwert $18 \%$ ). Die Auswertung zeigt, dass die Bereitstellung von Marktinformationen das dominierende Produkt des TecNet darstellt.

Die Marktinformationen des TecNet werden bei den Kunden größtenteils zur Abschätzung des Marktpotenzials verwertet (81 \%). Immerhin 44 Prozent der Auftraggeber nutzt die Dienstleistung des TecNet um das Wettbewerbsumfeld einzuschätzen (vgl. Tabelle 10).

Tabelle 10: $\quad$ Nutzung der Marktinformationen des TecNet

\begin{tabular}{lll}
\hline Marktinformationen des TecNet nutzten Sie... & abs. & in \% \\
\hline zur Abschätzung des Marktpotenzials & 13 & 81 \\
zur Erkundung potenzieller Mitbewerber & 7 & 44 \\
zur Präsentation/Vermarktung von Projekten & 2 & 13 \\
für die Erstellung von Business-Plänen etc. & 3 & 19 \\
\hline
\end{tabular}

Mehrfachantworten möglich

Insgesamt wird das Kosten/Nutzen-Verhältnis von zwölf Mandanten des TecNet (75 \%) als ,fair“ bezeichnet. Zwei Kunden beurteilen diese Relationen sogar als ausgezeichnet. Ebenfalls erfreulich ist die hohe Informationsqualität, die das TecNet seinen Kunden offeriert. $63 \%$ der Kunden beurteilen die Qualität als ,gut“; vier Kunden (25\%) vergeben sogar die Bestnote. Die Informationsdienstleistungen des TecNet werden wegen ihrer hohen Informationsqualität auch gewinnbringend bei den Auftraggebern genutzt. 
Sechs Mandanten geben an, dass die Informationen von Ihnen zu $100 \%$ genutzt wurden, d.h. vollständig in Entscheidungsprozesse eingegangen sind. Weitere fünf Unternehmen nutzten die gelieferten Informationen noch $\mathrm{zu}$ mindestens $75 \%$. Diese hohe Nutzungsfrequenz zeigt, dass die Leistungen des TecNet nicht nur angefragt sondern auch verwertet werden.

Die notwendigen Abstimmungsprozesse zwischen Auftraggeber und TecNet verlaufen problemlos. Alle Kunden beurteilen die Gespräche als „gut“ bis „sehr gut“. Der professionelle Gesamteindruck des Projektmanagements gewährleistet somit auch ein zukünftiges Kundenpotenzial: bis auf ein Unternehmen würden die Klienten dieser Befragung die Dienstleistungen des TecNet wiederholt in Anspruch nehmen. Mit fünf Unternehmen bzw. Ansprechpartnern existiert bereits eine regelmäßige Kooperation; in sieben Fax-Antworten wurde eine Kooperation als ,,wünschenswert“ angekreuzt.

Auf die Frage, ob das TecNet seine Projekte denn auch termingetreu erfüllt, antworteten zwölf Firmen (75\%) mit ,ja“" und auch die verbleibenden Unternehmen hatten mit „akzeptabel“ keine Beschwerden vorzubringen.

\section{Die Informationsbeschaffung österreichischer Unternehmen}

An der schriftlichen Befragung zur Bekanntheit des TecNet beteiligten sich 66 technologieorientierte Firmen, die bislang noch keine Dienste des TecNet in Anspruch genommen haben. Nahezu die Hälfte dieser Unternehmen sind kleine und mittlere Firmen mit weniger als 50 Beschäftigten. $17 \%$ aller antwortenden Unternehmen sind Unternehmensgründungen. Der Großteil dieser Firmen ist eigenständig und im Unterschied zu den Großunternehmen des Samples nicht Teil einer Unternehmensgruppe.

Der Grad der Internationalisierung ist hoch. Nur zehn Prozent der befragten Unternehmen bieten ihre Produkte ausschließlich in Österreich an. Alle anderen Firmen vertreiben ihre Produkte und Dienstleistungen auch in anderen europäischen Ländern (44 \%) oder sogar weltweit (46\%).

Abbildung 5: Unternehmensgröße und Alter

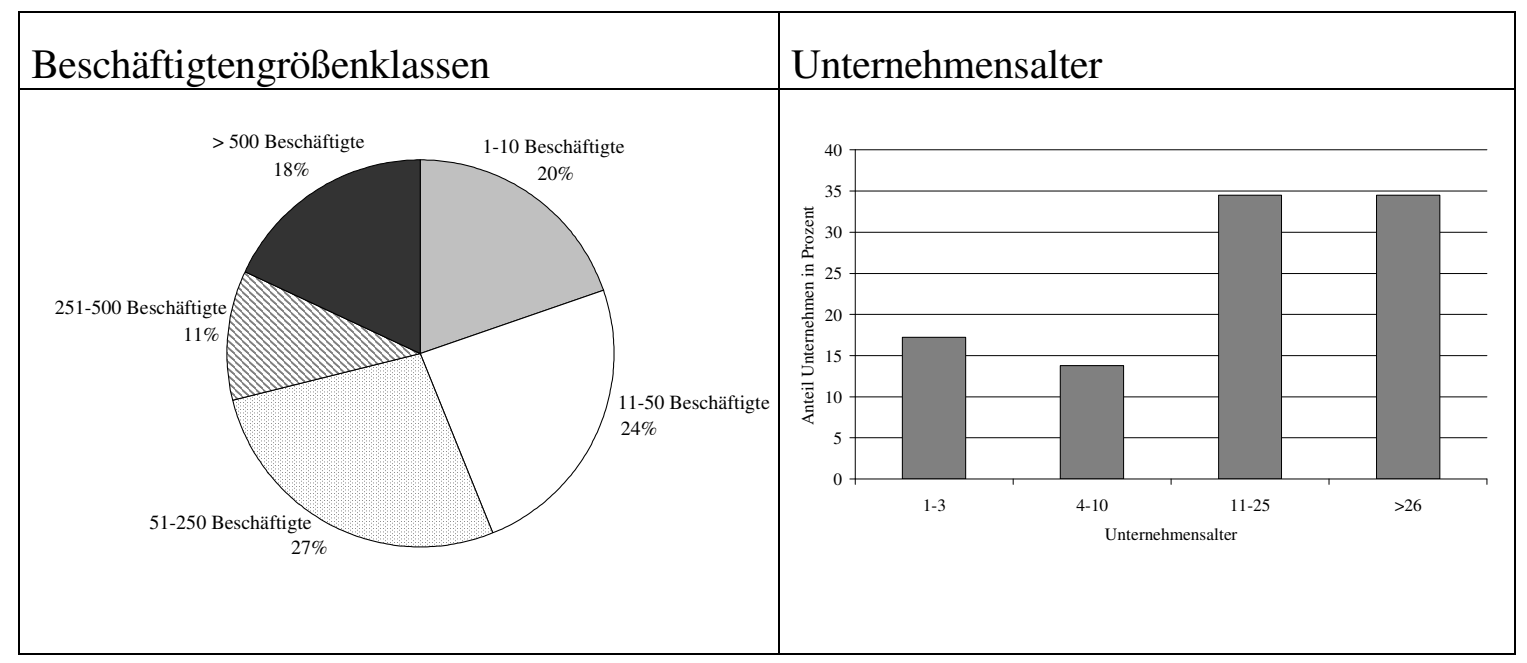


Ein Ziel der Befragung war es, die allgemeine Bekanntheit des TecNet zu ermitteln. Da das TecNet eine Organisationseinheit der Innovationsagentur ist, wurde sowohl nach der Bekanntheit der Innovationsagentur, als auch nach der Bekanntheit des TecNet gefragt (vgl. Tabelle 11).

Tabelle 11: Bekanntheit der Innovationsagentur und des TecNet

\begin{tabular}{|c|c|c|c|c|c|}
\hline \multirow{2}{*}{\multicolumn{2}{|c|}{$\begin{array}{l}\text { Bekanntheit... } \\
\text { (Sitz in Wien) }\end{array}$}} & \multicolumn{2}{|c|}{ der Innovationsagentur } & \multicolumn{2}{|c|}{ des TecNet } \\
\hline & & abs. & $\%$ & abs. & $\%$ \\
\hline \multicolumn{2}{|c|}{ Unternehmensgründungen* } & $2(2)$ & $14(25)$ & 0 & 0 \\
\hline \multicolumn{2}{|c|}{ KMU } & $3(1)$ & $21(13)$ & $2(1)$ & $25(33)$ \\
\hline \multicolumn{2}{|c|}{ Großunternehmen } & $4(0)$ & $29(0)$ & $4(0)$ & $50(0)$ \\
\hline \multicolumn{2}{|c|}{ Wissenschaftseinrichtungen $* * *$} & $5(5)$ & $36(63)$ & $2(2)$ & $25(77)$ \\
\hline \multicolumn{2}{|l|}{ Summe } & $14(8)$ & $100(100)$ & $8(3)$ & $100(100)$ \\
\hline merkungen: & $\begin{array}{ll}* & \text { Personen im Grün } \\
* * & \text { Geldgeber und et } \\
* * * & \text { Universitätsanges }\end{array}$ & $\begin{array}{l}\text { lierte U } \\
\text { llte, Be }\end{array}$ & amen jüng & ahre & \\
\hline
\end{tabular}

Die Auswertungen zeigen, dass beide Einrichtungen den meisten Unternehmen bzw. Wissenschaftseinrichtungen unbekannt sind: dies gilt für die Innovationsagentur bei $77 \%$ und für das TecNet bei $86 \%$ der Befragten. Dabei ist festzustellen, dass von acht Firmen, denen das TecNet ein Begriff ist, immerhin sieben Firmen auch die Innovationsagentur kennen. Umgekehrt geben allerdings nur sechs von 14 Unternehmen an, das ihnen zwar die Innovationsagentur, nicht aber dass TecNet bekannt ist. Diesen Angaben entsprechen der Erwartung, dass sich die Innovationsagentur stärker als Unternehmen etabliert hat, als das noch junge TecNet als „Marke“ bzw. als Leistungsträger dieses Unternehmens.

Da die Innovationsagentur ihren Unternehmenssitz in Wien hat, ist sie, wie auch das TecNet, überwiegend denjenigen Firmen/Forschungseinrichtungen bekannt, die ebenfalls in Wien ansässig sind. Hier ist persönlichen Kontakten bzw. der räumlichen Nähe eine wichtige Marketing-Funktion beizumessen. In dieser Befragung findet sich in den Textfeldern (,,bekannt durch...?“) überwiegend der Eintrag ,,persönliche Kontakte“ und „Medien“ sowie „Innovationspreis“. Beim TecNet finden sich als Texteinträge: „IT/Medien“, ,Internet“, „Homepage“ und „Vorträge“.

Auch wenn die Ergebnisse dieser Umfrage wegen des geringen Stichprobenumfangs nicht repräsentativ für alle Unternehmen in Österreich sind, so zeigt sich doch, dass beide Einrichtungen vor allem von der Wissenschaft und größeren Unternehmen wahrgenommen werden. Unternehmensgründer und KMU hingegen sind mit dem Angebot der Innovationsagentur und des TecNet wenig vertraut.

Ein anderer Fragenblock der Kurzbefragung fokussiert die Quellen der Informationsbeschaffung österreichischer Unternehmen. Hier wird der Frage nachgegangen, über welche alternativen Informationsquellen wie z.B. Kunden, Bankberater, Informationsbroker etc. sich eine Firma Informationen beschafft.

Die Umfrageergebnisse zeigen, dass sich der weitaus größte Teil der Firmen seine Marktinformationen aus erster Quelle, das heißt von Zulieferern und Kunden beschafft. Insgesamt 54 Unternehmen (82\%) wählen somit die Direktansprache, wobei sie auf ihren 
Vertrieb (53\%) und auf eigene Datenbanken bzw. Recherchen (52\%) zurückgreifen (vgl. Tabelle 12).

Tabelle 12: $\quad$ Recherche von Marktinformationen

\begin{tabular}{lll}
\hline Marktinformationen beschaffen Sie durch... & abs. & in \% \\
\hline Zulieferer und Kunden (Direktansprache) & 54 & 82 \\
Ihren Vertrieb & 35 & 53 \\
geschulte Mitarbeiter/eigene Abteilung & 23 & 35 \\
eigene Datenbank-/Internet- Recherchen & 34 & 52 \\
externe Berater/spezialisierte Firmen & 11 & 17 \\
Teilnahme an Messen/Tagungen/Verband etc. & 45 & 68 \\
Experten Ihrer Bank/Venture Capital Gesellschaft & 5 & 8 \\
\hline
\end{tabular}

Mehrfachantworten möglich

Eine besondere Bedeutung bei der Beschaffung von Marktinformationen wird auch der Teilnahme an Messen und Fachveranstaltungen beigemessen. Mehr als zwei Drittel aller befragten Firmen gibt an, dass sie sich auf diesen Marktplätzen Informationen über das Angebot ihrer Mitbewerber verschafft. Dagegen spielen Experten der Bank eine untergeordnete Rolle. Von ihren Kapitalgebern bzw. Kreditinstituten erfahren lediglich fünf Unternehmen ( $8 \%$ ) Informationen und Trends, die ihren Markt betreffen.

Bei der Analyse der Beschaffung von Technologieinformationen zeigt sich, dass - wie bereits bei den Marktinformationen festgestellt - auch hier die Fachmessen und Produktpräsentationen von großer Bedeutung sind. Insgesamt 50 der antwortenden Unternehmen (76 \%) geben an, dass sie Technologieinformationen über Messen, Fachtagungen oder Fachzeitschriften recherchieren.

Tabelle 13: Recherche von Technologieinformationen

\begin{tabular}{lll}
\hline Technologieinformationen beschaffen Sie durch... & abs. & in \% \\
\hline eigene Datenbank-/Internet Recherchen & 33 & 50 \\
Kooperationspartner (Unis, andere Firmen etc.) & 44 & 67 \\
Teilnahme an Messen/Fachtagungen, Zeitschriften & 50 & 76 \\
spezialisierte Mitarbeiter (Ingenieure, Techniker) & 40 & 61 \\
externe Berater/spezialisierte Firmen & 13 & 20 \\
Patentamt, Patentanwälte & 8 & 12 \\
eigene Abteilung für Research/Forschung & 19 & 29 \\
\hline
\end{tabular}

Mehrfachantworten möglich

Eine ebenso bedeutende Informationsquelle sind Kontakte zu Kooperationspartnern, die mit den, für die Firmen relevanten, Technologien in Verbindung stehen. Über zwei Drittel der Befragten beschafft sich Technologieinformationen über Kooperationspartner, zu denen Universitäten, Fachhochschulen aber auch andere Unternehmen zählen.

Das TecNet kann seine Dienstleistungen am besten dort anbieten, wo seine Kunden mit Hemmnissen der Informationsbeschaffung konfrontiert sind. Die Faxumfrage zeigt, dass 
insbesondere eine systematische Recherche zu den größten Problemen der Unternehmen zählt. $42 \%$ der Befragten geben an, dass sie Rechercheprobleme haben, weil das Informationsangebot unübersichtlich sei. Diese führt häufig zu einer unsystematischen Suche z.B. in Datenbanken und im Internet und ist für viele Firmen extrem zeitaufwendig. Vielen Unternehmen (36\%) fehlen für diese Aufgaben die entsprechenden Ressourcen. Der Suchaufwand $(27 \%)$ wird als hoch eingeschätzt und ist im Hinblick auf ihren ursprünglichen Informationsbedarf nur selten erfolgreich.

Tabelle 14: $\quad$ Hemmnisse der Informationsbeschaffung

\begin{tabular}{lcc}
\hline $\begin{array}{l}\text { Die größten Probleme ergeben sich bei Ihren eigenen } \\
\text { Informationsrecherchen... }\end{array}$ & abs. & in \% \\
\hline weil wichtige Infos nicht veröffentlicht werden & 26 & 39 \\
weil Ihre Kapazitäten nicht ausreichen & 24 & 36 \\
weil die Konkurrenz Ihre Pläne erkennen kann & 1 & 2 \\
weil Datenbankinformationen häufig veraltet sind & 15 & 23 \\
weil das Informationsangebot unübersichtlich ist & 28 & 42 \\
weil spezifische Infoquellen zu teuer sind & 16 & 24 \\
durch die hohen ,Suchkosten“ (Aufwand) & 18 & 27 \\
\hline
\end{tabular}

Mehrfachantworten möglich

Knapp $40 \%$ der befragten Unternehmen ist der Ansicht, dass die für sie wichtige Informationen überhaupt nicht veröffentlicht werden und für $23 \%$ sind Informationsrecherchen problematisch, weil die Daten aus ihrer Sicht veraltet sind. Qualitativ hochwertige Informationen erscheinen immerhin einem Viertel der antwortenden Unternehmen als zu teuer. Dieses Antwortverhalten zeigt, dass sich dem TecNet gerade bzgl. der Aktualität und des Kostenbewusstseins gute Vermarktungschancen bieten.

Im Fragenblock der Hemmnisse werden auch die Leistungen von Informationsbrokern und somit indirekt das Leistungsangebot des TecNet - abgefragt. Dabei zeigt sich, dass Informationsbrokern eine wichtige Rolle im „Informationsdschungel“ beigemessen wird.

\section{Tabelle 15: Bedeutung von Informationsbrokern}

\begin{tabular}{lll}
\hline Informationsbroker... & abs. & in \% \\
\hline sollten mehr auf sich aufmerksam machen & 32 & 48 \\
sind eine Alternative zu teuren „Consultants“ & 9 & 14 \\
helfen vor allem Unternehmensgründern/Start-Ups & 6 & 9 \\
verfügen über zu geringes technisches Know-how & 10 & 15 \\
werden im Internet-Zeitalter immer entbehrlicher & 18 & 27 \\
gewährleisten keine bessere „Informationsqualität“ & 10 & 15 \\
kennen Ihre Geschäftsfelder/Markt nicht gut genug & 14 & 21 \\
\hline
\end{tabular}

Mehrfachantworten möglich

Nahezu die Hälfte aller befragten Firmen wünschen sich, dass „Infobrokern“ mehr auf sich aufmerksam machen. Hier ist $\mathrm{zu}$ vermuten, dass bei vielen Unternehmen Unsicherheiten über das Dienstleistungsangebot, die Recherchemöglichkeiten und die 
Kosten bestehen. Zwar sind die Firmen in der Lage, zwischen „Consultants“ (externen Beratern) und Informationsdienstleistern zu unterscheiden, das Spektrum der Produkte eines Infobrokers ist ihnen jedoch kaum bekannt. Für diese Einschätzung spricht auch, dass $27 \%$ der Befragten den Informationsbroker wegen verbesserter InternetTechnologien in Zukunft für immer entbehrlicher halten.

Vielfach besteht die Vorstellung, dass ein Informationsbroker lediglich im Internet „surft“, kostenlose Daten sammelt und diese dann verkauft. Das Leistungsbewusstsein, dass ein seriöser Infobroker, wie das TecNet, qualitativ hochwertige Datenbanken (gerade) auch außerhalb des Internets abfragt, Analysen von Experten beschafft und zielgerichtet auswertet, ist vielfach noch nicht ausgeprägt. Weitere Belege für diese Einschätzung ergeben sich aus daraus, dass $15 \%$ der antwortenden Firmen die Auffassung vertreten, dass Informationsbroker über ein zu geringes technisches Knowhow verfügen, um effizient recherchieren zu können. $21 \%$ sind der Meinung, dass man die Geschäftsfelder bzw. den Markt ihres Unternehmens nicht gut genug kennt.

\section{Kunden und Nicht-Kunden im Vergleich}

Anlass der Unternehmensbefragung war es u.a. festzustellen, welchen Stellenwert externe Informationsdienstleistungen bei privaten Firmen einnehmen. Die Einschätzung, ob sich das Dienstleistungsangebot des TecNet überholt hat, oder ob dieses Angebot auf ein beständiges oder sogar zunehmendes Interesse stößt, ist für die Beurteilung der Zukunftsperspektiven des TecNet elementar. Aus diesem Grund wurden sowohl die bisherigen Kunden als auch die potenziellen zukünftigen Mandanten des TecNet nach der Relevanz von externen Informationsdienstleistungen für ihre Unternehmung gefragt.

Tabelle 16: Relevanz „externe Informationsdienstleistungen“

\begin{tabular}{lllll}
\hline Externe Informationsdienstleistungen... & \multicolumn{2}{c}{ TecNet-Kunden } & \multicolumn{2}{c}{ Nicht TecNet-Kunden } \\
& abs. & in \% & abs. & in \% \\
\hline sind für Sie heute nicht mehr relevant & 0 & 0 & 13 & 20 \\
werden für Sie zunehmend wichtig & 10 & 63 & 11 & 17 \\
sind eine Alternative zu eigenen Recherchen & 8 & 50 & 14 & 21 \\
sind eine Ergänzung zu eigenen Recherchen & 9 & 56 & 32 & 48 \\
sind eher für Gründer/Start-Ups von Bedeutung & 0 & 0 & 10 & 15 \\
sollten kommerziellen Firmen überlassen bleiben & 0 & 0 & 3 & 5 \\
\hline
\end{tabular}

Insgesamt $48 \%$ der Unternehmen, die noch keine Leistungen des TecNet in Anspruch genommen haben, sehen die Informationsdienste als eine Ergänzung ihrer eigenen Recherchen. Das TecNet wird von diesen Firmen als Spezialist betrachtet, den man beauftragt, wenn man bereits eine Vorstellung von Markt- bzw. Technologieinformationen hat. Die Unternehmen betrachten die Leistungen eines externen Informationsdienstleisters in der Regel als ,,add-on“ (Mehrwert) und seltener als Alternative (21\%) zu eigenen Recherchen.

Die mangelnden Kenntnisse über die Dienste und Produkte eines externen Informationsdienstleisters spiegeln sich auch in der Einschätzung der künftigen Relevanz für ein

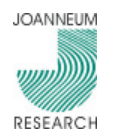


Unternehmen wider. Während die meisten Kunden des TecNet die Leistungen für zunehmend wichtig halten (63\%), beurteilen lediglich $17 \%$ der Nicht-Kunden ein solches Angebot für zunehmend relevant. Insbesondere Finanzdienstleister (Kreditinstitute, Förderbanken etc.) profitieren vom TecNet-Angebot und zählen z.T. schon zu den Stammkunden. Die meisten der ehemaligen Kunden, so wurde bereits ausgeführt, würden bzw. beauftragen das TecNet auch heute wieder. Kurz: Wurde erst einmal die Leistung durch das TecNet erbracht, haben die Kunden eine konkrete Vorstellung vom gelieferten Produkt und seiner Qualität. Es muss angenommen werden, dass sich im Zuge der geleisteten Dienste auch die zuvor skeptischen Einschätzungen der Kunden ändern.

Im abschließenden Fragenblock der Erhebung wurden die Unternehmen aufgefordert, ihre eigenen Informationsmöglichkeiten mit denen eines Informationsbrokers zu vergleichen (Tabelle 17).

\section{Tabelle 17: $\quad$ Vorteile von Informationsbrokern}

\begin{tabular}{lcccc}
\hline $\begin{array}{l}\text { Informationsbroker haben im Unterschied zu } \\
\text { Ihrem Unternehmen... }\end{array}$ & \multicolumn{2}{c}{ TecNet-Kunden } & \multicolumn{2}{c}{ Nicht TecNet-Kunden } \\
& abs. & in \% & abs. & in \% \\
\hline weitreichendere Ressourcen & 8 & 50 & 16 & 24 \\
spezifische Rechercheerfahrung/-techniken & 11 & 69 & 23 & 35 \\
Zugriff auf kostspielige Analysen/Berichte & 13 & 81 & 16 & 24 \\
besseren Zugang zu Experten-Netzwerken & 5 & 31 & 21 & 32 \\
mehr Zeit, um sich Recherchen zu widmen & 8 & 50 & 29 & 44 \\
aktuelle Informationen verfügbar & 2 & 13 & 4 & 6 \\
bessere Kontakte zur Wissenschaft & 2 & 13 & 9 & 14 \\
\hline
\end{tabular}

Auch hier zeigt sich, dass deutliche Unterschiede zwischen den Kunden des TecNet und den Nicht-Kunden bestehen: Während von den Kunden konkrete Leistungen (Zugriff auf kostspielige Analysen/Berichte: $81 \%$, spezifische Rechercheerfahrung: $69 \%$ ) des TecNet als Vorteil gegenüber ihrer Firma angesehen werden, dominiert bei den Nicht-Kunden die Vorstellung, dass Informationsbroker vor allem „mehr Zeit“ (44\%) mitbringen. Dieses Antwortverhalten ist als weiteres Indiz zu deuten, dass vielen Unternehmen nur eine diffuse Vorstellung vom Gesamtangebot eines Informationsbrokers haben.

Bei den Angaben der befragten Unternehmen erstaunt, dass nur ein geringer Anteil (13\% TecNet-Kunden, $6 \%$ Nicht-TecNet Kunden) der Meinung ist, dass Informationsbroker über aktuellere Informationen verfügen als ihr eigenes Unternehmen. Im Allgemeinen wird von Informationsbrokern erwartet, dass sie zwar umfassender, nicht aber aktueller recherchieren als das Unternehmen selbst.

\subsubsection{Ergebnisse der Expertenbefragung}

Neben den an anderer Stelle dokumentierten Gesprächen mit Programmmanagement und Ministerien wurde seitens des Projektteams versucht, einen Eindruck zu gewinnen, welche Positionierung „TecNet“ in der österreichischen Förderlandschaft einnimmt. Dies geschah mit folgenden Hintergedanken: TecNet firmiert einerseits als „Netzwerk“. In 
seinem Selbstverständnis definiert das TecNet den Netzwerkgedanken als Kooperation mit Landesorganisationen, dem Wifi oder AplusB-Zentren. Andererseits sind Unternehmensgründer eine wesentliche Zielgruppe des Programms, wobei TecNet ja nicht den gesamten Gründungsprozess begleitet, sondern eine bestimmte Hilfestellung anbietet. Folglich war die Einschätzung von TecNet durch das Management verschiedener Gründerprogramme ein Mittel, die Einbindung in die Förderlandschaft Österreichs und die Bedeutung von TecNet für Unternehmensgründer zu beurteilen.

Es wurden Österreichweit 13 einschlägige Programme identifiziert und 19 Programmmanager per email angeschrieben (vgl. Materialien 10 und 11). Insgesamt beantworteten sechs angeschriebene Personen den Fragebogen, mit zwei Personen kam es zu längeren telefonischen Gesprächen.

Insgesamt entsteht der Eindruck, dass sowohl die Innovationsagentur als auch „TecNet“ den Programmmanagern bekannt sind. Diese „Bekanntheit“ erstreckt sich von einer bloßen Geläufigkeit des Programms bis hin zu intensiver Kooperation. Gemessen an den jeweiligen Aufgaben wird TecNet überwiegend als „willkommener Netzwerkpartner“ beurteilt.

Auf die Frage, welchen Stellenwert das TecNet aus Sicht des Befragten in der Gründungs- und Förderlandschaft einnimmt, wird TecNet einhellig als „interessante Dienstleistung im Kontext mit Gesamtpaketen“, als interessante "Zweit-Anlaufstelle“ eingeschätzt. Als positiv werden die High-Tech-Kompetenzen des TecNet bewertet. Wenn es zu Kooperationen kommt, dann als „Ergänzung zur Beurteilung von High-Tech Projekten“ bzw. als „Ergänzung im High-Tech-Bereich.“ Die Qualität der gelieferten Informationen in diesen Kooperationen wird als hoch eingeschätzt. Betont wird, dass das Angebot von TecNet vor allem für Start-Ups interessant ist, für die TecNet die Einschätzung ihrer Marktchancen erleichtert, weniger für etablierte Unternehmen. Bemängelt wird (und das sind Einzeleindrücke der Befragten) der „geringe Bekanntheitsgrad" von TecNet, der schwierige Zugang und die hohen Kosten; skeptisch wird beurteilt, in wie weit Hilfestellungen bei sehr spezifische Markt- und Technologieinformationen gegeben werden kann.

\subsection{FAZIT}

Der Anteil technologieorientierter Unternehmensgründungen ist in Österreich zwischen 1997 und 2000 angestiegen und wird im vor allem von der Dynamik des Dienstleistungssektors geprägt. Die Marktbedingungen des TecNet waren in diesen Jahren besonders günstig, weil das allgemein positive Gründungsklima an den Kapitalmärkten dazu führte, dass sowohl Unternehmensgründer als auch Kapitalgeber die Dienste des TecNet in Anspruch nahmen. Seit Ende 2000 hat die Gründungseuphorie, die nicht zuletzt von den Erwartungen der Investoren (Business Angels, Kreditinstitute und VC-Gesellschaften) beflügelt wurde, merklich nachgelassen. Es ist daher damit zu rechnen, dass sich der positive Gründungstrend bei technologieorientierten Unternehmen abschwächt. Die Potenzialanalyse dieser Studie geht jedoch davon aus, dass in Österreich nach 2001 auch 
weiterhin mindestens 1.300 technologieintensive Unternehmen pro Jahr gegründet werden. Diese Unternehmen bilden neben den etablierten Firmen das Marktpotenzial des TecNet. Der Standort Wien kommt dem TecNet zugute, da der Großraum Wien die höchsten Gründungsraten im gesamten österreichischen Bundesgebiet aufweist. Angesichts dieser Prognosen und in Anbetracht eines mäßigen Wettbewerberumfeldes sind die zukünftigen Vermarktungschancen der TecNet-Dienstleistungen als gut einzuschätzen.

In der schriftlichen Befragung dieser Evaluation wurden insgesamt 219 Unternehmen, Forschungseinrichtungen und Förderinstitutionen kontaktiert. Von diesen antworteten 82 Gesellschaften, was einem Rücklauf von $37 \%$ entspricht. 16 Firmen sind TecNetKunden $^{8}$; 66 Firmen sind keine Kunden. Über zwei Drittel dieser Firmen sind kleine und mittlere Unternehmen mit weniger als 250 Mitarbeiter die ihre Produkte international vertreiben.

Die Kunden des TecNet geben an, nahezu ausschließlich Marktinformationen angefragt und erhalten zu haben. Diese Marktinformationen wurden von $81 \%$ der Klienten zur Abschätzung ihres Marktpotenzials verwertet. Das Kosten/Nutzen-Verhältnis wird als „fair“ oder sogar ,ausgezeichnet“ bezeichnet. Auch bei der Qualität sind die Kunden des TecNet außerordentlich zufrieden: $63 \%$ der Kunden beurteilen die Informationsqualität als „gut“; vier Kunden (25\%) vergeben sogar die Bestnote. Diese Qualitätsmerkmale veranlassten sechs Mandanten die gelieferten Informationen vollständig in ihren Entscheidungsprozessen zu berücksichtigten. Weitere fünf Unternehmen geben an, dass sie die Informationen noch zu mindestens $75 \%$ verwenden konnten. Diese hohe Nutzungsfrequenz zeigt, dass die Leistungen des TecNet nicht nur angefragt sondern auch verwertet werden. Darüber hinaus wird dem TecNet ein professionelles Management attestiert, welches dazu beiträgt, dass sich die meisten Firmen eine regelmäßige Kooperation wünschen. Bis auf eine Ausnahme, würden alle bisherigen Kunden das TecNet wiederholt mit einem Rechercheauftrag betrauen.

Trotz dieser Kundenzufriedenheit ist das TecNet den meisten technologierorientierten Unternehmen und Kapitalgebern in Österreich unbekannt: von 66 zufällig in Österreich ausgewählten technologieintensiven Unternehmen, Kapitalgebern und Wissenschaftseinrichtungen geben $86 \%$ an, dass sie das TecNet nicht zu kennen. Anhand dieser (nicht repräsentativen) Befragung zeigt sich, dass sich das TecNet noch nicht als „Marke“ bzw. als Leistungsträger der Innovationsagentur etablieren konnte. Insbesondere Unternehmensgründern und KMU ist das TecNet als Institution neu.

Die Auswertungen zeigen, dass sich die meisten Firmen ihre Marktinformationen direkt bei Kunden und Zulieferern, z.B. über ihren Vertrieb beschaffen. Ein weiterer bedeutender Informationspool für Technologie- und Marktinformationen sind Messen und Fachveranstaltungen. Angesichts dieser Umfrageergebnisse wäre dem TecNet - aus Marketinggesichtspunkten heraus - eine verstärkte Teilnahme an Messen zu empfehlen. Die meisten Firmen (42\%) sehen sich bei der Informationsbeschaffung einem unübersichtlichen Informationsangebot gegenüber. Die unsystematische Suche in Datenbanken

${ }^{8}$ Präzise handelt es sich um 16 Faxantworten von 14 verschiedenen Kunden (Unternehmen) des TecNet. 
und im Internet ist für viele Firmen problematisch und zeitintensiv. Viele ,wichtige“ Informationen, so geben $36 \%$ der Firmen an, werden erst gar nicht veröffentlicht. Nahezu die Hälfte aller antwortenden Firmen wünschen sich, dass Informationsbroker mehr auf sich aufmerksam machen. In diesem Zusammenhang ist festzustellen, dass die Leistungen eines Informationsbrokers (qualitativ hochwertige Datenbanken und Studien zu recherchieren und diese in Bezug auf den Kundenauftrag hin zu bewerten), vielfach noch unbekannt sind.

Die mangelnden Kenntnisse über die Dienste und Produkte eines Informationsdienstleisters spiegeln sich auch in der Einschätzung der künftigen Relevanz für die Unternehmen wieder. Während die meisten Kunden des TecNet die Leistungen für zunehmend wichtig halten (63\%), beurteilen lediglich $17 \%$ der Nicht-Kunden ein solches Angebot für zunehmend relevant. Insbesondere Finanzdienstleister (Kreditinstitute, Förderbanken etc.) profitieren vom TecNet-Angebot und zählen z.T. schon zu den Stammkunden. Es ist anzunehmen, dass im Zuge der geleisteten Dienste auch die Akzeptanz der Kunden zum Angebot eines Informationsbrokers zunimmt.

Grundsätzlich betrachten die meisten Unternehmen die Leistungen eines Informationsbrokers als Ergänzung der eigenen Recherchen (56 \% TecNet-Kunden; $48 \%$ NichtTecNet-Kunden). Insbesondere die Nicht-TecNet-Kunden sehen in diesem Angebot keine Alternative $\mathrm{zu}$ eigenen Recherchen. Ein Informationsbroker, so die verbreitete Auffassung, kann zwar umfassender nicht aber aktueller recherchieren als das Unternehmen selbst. 


\section{Programm- und Institutionenvergleich}

In diesem Kapitel wird die Dienstleistung des TecNet zunächst ähnlich gelagerten Maßnahmen des Bundes gegenübergestellt, um eine Duplizität öffentlicher Förderaktivitäten zu prüfen. Anschließend wird das Benchmarking als Instrument der Wettbewerbsanalyse eingesetzt, um Vorteile und Defizite des TecNet mit Referenzorganisationen zu identifizieren. Für den Dienstleistungsvergleich finden sich ein dem TecNet ähnliches Angebot in den „Feasibility“ Fördermaßnahmen des Forschungsförderungsfonds für die gewerbliche Wirtschaft (FFF). Als internationales Referenzbeispiel für das Benchmarking wurde der „RechercheDienst“ des deutschen Fachinformationszentrums Karlsruhe ausgewählt.

Grundidee des Vergleichs, wie auch des Benchmarking ist es, festzustellen, welche Gemeinsamkeiten und Unterschiede bei anderen Programmen bzw. Organisationen bestehen und welche Verbesserungsoptionen sich eröffnen. Diese Verbesserungsmöglichkeiten betreffen zum einen das TecNet als Teil eines umfassenden Maßnahmenbündels der Wirtschafts- und Innovationspolitik; zum anderen geht es um effizientere Organisationsstrukturen und Arbeitsabläufe im Aufgabenbereich des TecNet selbst.

\subsection{DER FORSCHUNGSFÖRDERUNGSFONDS FÜR DIE GEWERBLICHE WIRTSCHAFT (FFF)}

Die Innovationsagentur mbH (Innovationsagentur) und der Forschungsförderungsfonds für die gewerbliche Wirtschaft (FFF) werden von der österreichischen Bundesregierung mit dem Ziel finanziert, innovationsfördernde Rahmenbedingungen für eine international wettbewerbsfähige Wirtschaft und Wissenschaft zu schaffen. Eine bedeutende Aufgabe beider Gesellschaften ist die Förderung junger Technologieunternehmen in der Gründungs- und Wachstumsphase. Diese Förderung leitet sich aus dem wichtigen Beitrag $\mathrm{ab}$, den junge technologieorientierte Unternehmen zur Modernisierung der Wirtschaftsstruktur und für einen qualifizierten Arbeitsmarkt leisten.

Die Maßnahmen des FFF und der Innovationsagentur setzen bei der Förderung zwar an unterschiedlichen Stellen an, verfolgen aber das gemeinsame Ziel junge High-Tech Unternehmen in ihrem Gründungs-, Innovations- und Wachstumsprozess zu begleiten. So fördert die Innovationsagentur, ebenso wie der FFF technologieorientierte Unternehmen vor und während der Gründungs- bzw. Aufbauphase. In der Innovationsagentur finden sich Förderbereiche wie SeedCapital und TecNet; im FFF werden die Programme "StartUp-Förderung" und „Feasibility-Studies“ angeboten. Beide Gesellschaften bieten ihrer Zielgruppe Netzwerke zu Experten sowie Informations- und Beratungsleistungen an, bei denen die Machbarkeit eines neuen Produkts bzw. Prozesses oder das Marktpotenzial im Mittelpunkt stehen. So werden vom FFF Studien (Feasibility Studies) zur technischen Machbarkeit einer Idee gefördert, die explizit auch das wirtschaftliche Umfeld (Märkte) 
berücksichtigen. Diese Studien und Recherchen zu Technologie- und Marktinformationen des FFF überschneiden sich auf den ersten Blick mit dem Angebot der Innovationsagentur (TecNet).

Die Förderung der Recherche nach Markt- und Technologieinformationen erfolgt im Fall des TecNet zwar durch eine institutionelle Förderung, während bei den „Feasibility Studies“ die Recherche nach Markt- und Technologieinformationen durch Projektförderung finanziert wird, der Fördergegenstand ist jedoch derselbe. Inwiefern es sich bei den Leistungen des FFF und des TecNet um ein komplementäres oder substitutives Förderangebot handelt, ist Gegenstand dieses Abschnitts.

\subsubsection{Organisation}

Der Forschungsförderungsfonds für die gewerbliche Wirtschaft (FFF) wurde 1968 mit dem Ziel gegründet, durch Unterstützung industrieller Forschung und Entwicklung die Wettbewerbsfähigkeit der österreichischen Wirtschaft sowohl im industriellen als auch im Dienstleistungssektor aktiv zu unterstützen. Am Sitz des Fonds in Wien sind insgesamt 37 MitarbeiterInnen beschäftigt. Der FFF wird vom Bund finanziert und ist dem Bundesministerium für Verkehr, Innovation und Technologie als Aufsichtsbehörde unterstellt. Er folgt dem Auftrag des Forschungs- und Technologieförderungsgesetzes und unterstützt den Wissens- und Technologietransfer durch innovationsgerichtete Fördermaßnahmen. Darüber hinaus hilft der FFF Unternehmen durch sachkundige Bewertung der Zukunftschancen ihrer Projekte und dient bei Bedarf als Know-howTransferstelle.

Obwohl das TecNet der Innovationsagentur keine technologische Bewertung von Projekten durchführt, so trägt es dennoch zur Bewertung der Finanzierung und damit der Zukunftschancen von Projekten entscheidend bei. Die von TecNet recherchierten und dokumentierten Informationen dienen Unternehmensgründern, Start-Ups und Kapitalgebern als Bewertungsgrundlage ihrer Entscheidungen.

\subsubsection{Ziele und Aufgaben}

Die Förderungsmaßnahmen des FFF zielen darauf hin, die Entwicklung neuer Produkte und Verfahren anzuregen, Produktionsstrukturen zu verbessern, Produktion und Exporte zu steigern und damit die Basis für Vollbeschäftigung und sozialen Wohlstand zu sichern. Der FFF hat in diesem Zielgedanken für Österreichs Wirtschaft mehrere Aktionslinien etabliert, zu denen auch die Initiativen „Start Up-Förderung“ und die „Feasibility Studies“ zählen. Die „Machbarkeitsstudien“, die Bestandteil beider Aktionslinien sind, haben das Ziel, kleinen und mittleren Unternehmen mit einem überschaubaren Kostenaufwand zu hochwertigen Analysen zu verhelfen. Auf diese Weise soll das innovatorische und technologische Potenzial österreichischer Unternehmen, Forschungsinstitute und Erfinder konsequent erschlossen werden.

Das TecNet der Innovationsagentur verfolgt den gleichen Grundgedanken: Unternehmen mit einem überschaubaren Kostenaufwand hochwertige Markt- und Technologieinformationen zur Verfügung zu stellen. 


\subsubsection{Zielgruppe}

Die Zielgruppe des FFF sind ausschließlich kleine und mittlere Unternehmen, während das TecNet über diesen Adressaten hinaus auch etablierte Großunternehmen aus der Industrie oder dem Kreditgewerbe als Kunden hat.

Der FFF fördert Feasibility Studien (Start Up-Förderprogramm) bei technologieorientierten Unternehmen in einem Alter von bis $\mathrm{zu}$ drei Jahren, mit maximal 50 Mitarbeitern, sieben Mio. $€$ Jahresumsatz, einer Bilanzsumme von maximal fünf Mio. $€$ und einer Beteiligungshöchstgrenze durch ein Großunternehmen von $25 \%$. Einen Antrag in der Förderlinie „Feasibility Studies“ können kleine und mittlere Unternehmen in einer Größe von bis zu 250 Mitarbeitern stellen.

Das TecNet steht dagegen prinzipiell allen Unternehmen, unabhängig von Alter, Größe, Umsatz und Beteiligungen mit seinen Recherchedienstleistungen zur Verfügung.

\subsubsection{Produkte}

Die Aktionslinie „Feasibility Studies“ adressiert das „,reiche Ideenpotenzial hinsichtlich neuer Produkte und Innovationen“ der österreichischen Unternehmen. Um die Unsicherheiten über die Machbarkeit solcher Ideen zu minimieren, werden vom FFF Studien zur technischen Machbarkeit dieser Ideen gefördert. Der FFF gibt ein Mindest-Set an Inhalt und Umfang dieser Studien vor, zu dem eine Realisierungsabschätzung, eine Kostenschätzung für die Umsetzung sowie Überlegungen zum wirtschaftlichen Umfeld und zur Rentabilität gehören.

Die Initiative „Start up Förderung“ wirbt mit den Slogans „Mehr Geld für Ihr Unternehmen“, „Wege zur Risikofinanzierung“ und „objektive Technologiebewertung“; sie adressiert technologieorientierte Unternehmen, nicht älter als drei Jahre und mit max. 50 Mitarbeitern. Es geht ,um eine verbesserte Art der Projektfinanzierung bzw. förderung, für technologieorientierte, junge Unternehmen“. Damit wir ein Ziel des Rates für Forschung und Technologieentwicklung unterstützt, eine „Stimulierung bei Unternehmensgründungen im Hochtechnologiebereich $\mathrm{zu}$ bewirken.“ Die Start Up Förderung umfasst eine Reihe von Maßnahmen: Feasibility Studien (analog zur Aktionslinie oben), Technology-Rating „Techrate ${ }^{\circledR}$ “ (Techrate ist als direkte Dienstleistung konzipiert, bietet ein Intensiv-Coaching in ,der heißen Phase der Planung bzw. Umsetzung neuer Technologien“ an und soll dem Unternehmer ermöglichen, die Umsetzungswahrscheinlichkeit einer Idee zu bewerten), den Treffpunkt Venture-Forum (Venture-Capital Meetings zur Zusammenführung von potenziellen Geldgebern und attraktiven Start-Ups) bzw. einen Besserungsschein (Umwandlung von FFF- Darlehen).

Das Dienstleistungsangebot des TecNet ist mit dem Angebot des FFF durchaus vergleichbar. In beiden Förderlinien geht es um eine ,,verbesserte Art der Projektfinanzierung“. Im Fall des TecNet liegt die Förderung in der Verbesserung der Finanzierungsentscheidung in Unternehmen (Finanzierung einer Produktneueinführung etc.) oder bei Kapitalgebern; im FFF ist es die Förderung der Finanzierungsentscheidung eines Technologieprojekts.

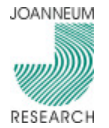




\subsubsection{Dienstleistung und Finanzierung}

Die Dienstleistungen des TecNet und die Inhalte der Feasibility Studies sind in ihrem Ablauf sehr ähnlich, unterscheiden sich jedoch hinsichtlich des Analysegegenstandes: Während beim TecNet die Recherche von technologischen Marktinformationen im Vordergrund steht, konzentriert sich der FFF auf Technologieinformationen. Das TecNet leistet vor allem Informationen zum wirtschaftlichen Umfeld. Es bereitet Marktinformationen auf, überlässt die Beurteilung der Informationen (Realisierungsabschätzung, Kostenabschätzung etc.) aber dem Unternehmen selbst. Eine externe Beurteilung eines Recherchegegenstandes findet nicht statt. In der Machbarkeitsstudie des FFF ist es die Technologie, die geprüft, im Hinblick auf ihre Realisierung und Kosten aber auch extern beurteilt wird. Das TecNet führt eine objektive Markteinschätzung durch, während der Auftragnehmer im Rahmen eines FFF-Programms eine Technologiebewertung praktiziert.

\section{Abbildung 6: Leistungsangebot der Initiative „Feasibility Studies“ und des TecNet}

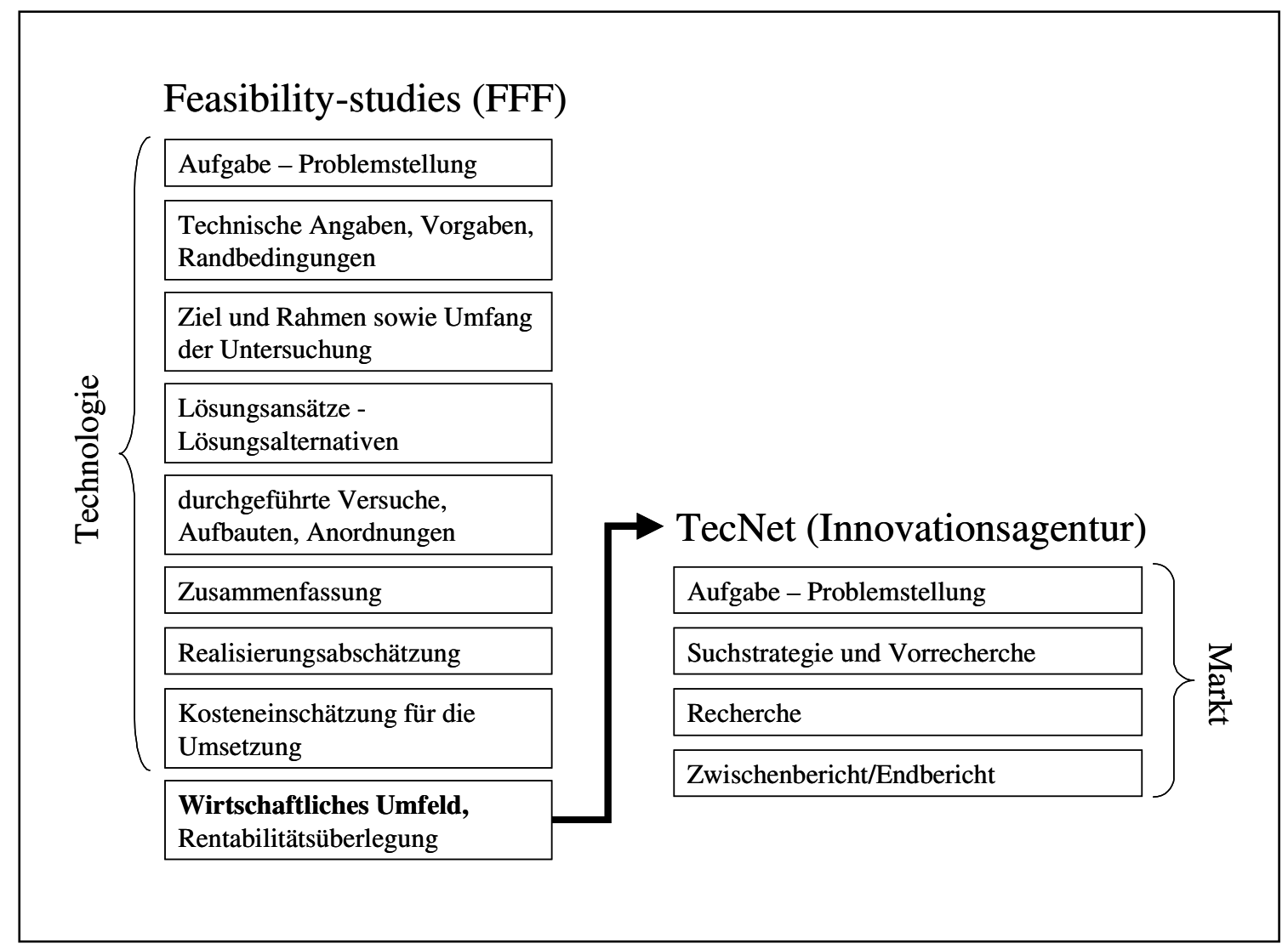

Die Kosten externer ExpertInnen (Universitätsinstitute, ACR, qualifizierte Sachverständige) der Machbarkeitsstudien werden im Programm Feasibility Studies zu $70 \%$ gefördert, wobei die Erstattungsgrenze mit $€ 8.000$ limitiert ist. Durch das Start Up Programm fördert der FFF Sach- und Personalkosten in der Höhe von maximal 50 \% der gesamten Projektkosten.

Die Infrastruktur des TecNet wird vom Bund und anderen Sozialpartnern finanziert. Die Förderung erfolgt hier nicht „direkt“ an ein Unternehmen, sondern ,,indirekt“ über das TecNet, das durch Zuschüsse des Staats seine Dienstleistungen preisgünstig anbieten 
kann. In beiden Förderangeboten sind Unternehmen jedoch verpflichtet, einen Eigenbeitrag für die Recherchen/Studien zu zahlen.

\subsubsection{Auftragsabwicklung}

Die Förderprogramme des FFF müssen von Unternehmen beantragt werden. Die Entscheidung, ob eine Firma in den Genuss der Förderung kommt, hängt davon ab, ob die Fördervoraussetzungen erfüllt sind. Der FFF steht mit seiner Kompetenz den anfragenden und geförderten Unternehmen zur Verfügung und hilft somit z.B. geeignete Einrichtungen/Experten für die Erstellung von Studien zu vermitteln.

Das TecNet steht grundsätzlich allen Unternehmen offen, die auf technologische Marktinformationen angewiesen sind. Die Auftragsvergabe ist zwar nicht wie beim FFF an Voraussetzungen gebunden, kann vom TecNet aber zielgruppenorientiert gesteuert werden. Sowohl das TecNet als auch der FFF müssen auf ihr Angebot durch Marketingaktivitäten, Fachreferate und Messepräsenz aufmerksam machen.

\subsubsection{Fazit}

Die institutionelle Förderung des TecNet und die Förderprogramme Start Up und Feasibility Studies des FFF weisen in ihren Zielen, bei der Zielgruppe sowie in der Finanzierungsform Gemeinsamkeiten auf, die zu der Annahme verleiten, es handle sich um ein substitutives Förderangebot. Beide Förderformen wollen dazu beitragen, Finanzierungsentscheidungen bei innovativen KMU, insbesondere bei technologieorientierten Start Up-Unternehmen durch bessere Informationsgrundlagen abzusichern. Darüber hinaus verlangen sowohl das TecNet als auch der FFF eine finanzielle Eigenbeteiligung ihrer Kunden zu den Gesamtkosten der Informationsbeschaffung.

Ein detaillierter Vergleich der beiden Maßnahmen zeigt jedoch entscheidende Unterschiede im Dienstleistungsschwerpunkt, so dass es sich zweifelsfrei um komplementäre Förderinstrumente handelt. Obwohl die technologiepolitischen Ausgangsüberlegungen die gleichen sind, werden in beiden Maßnahmen unterschiedliche Informationsinhalte erhoben. Während sich TecNet in den vergangenen zwei Jahren auf die Recherche von Marktinformationen konzentriert hat, ist die Förderung des FFF auf die Prüfung und Umsetzung einzelner Technologien ausgerichtet. Das TecNet deckt mit seinem Leistungsangebot somit die ,wirtschaftlichen Perspektiven“ eines Finanzierungsprojekts ab, während der FFF mit seinen Feasibility Studien die „technische Realisierung“ auslotet. Die Prüfung des wirtschaftlichen Umfeldes als Bestandteil der Machbarkeitsprüfung ist beim FFF lediglich eine von mehreren Positionen der Studie. Das TecNet hat sich hingegen auf detaillierte Marktrecherchen spezialisiert und gewährleistet Informationen für einen umfassenden Marktüberblick.

Eine Kooperation des TecNet mit Forschungsinstitutionen, die Studien im Rahmen der FFF-Förderung für Unternehmen durchführen, wäre aus innovationspolitischer Sicht wünschenswert. Durch die Vernetzung erlangen spezialisierte Forschungseinrichtungen nicht nur den Einblick in das technologische Potenzial einer Erfindung, sondern werden auch auf die Bedeutung der Absatzmärkte hingewiesen. Hier sind auf Seiten der 
theorielastigen Forschungsinstitute Lern- und Erfahrungseffekte zu erwarten, weil zukünftig bei der Beurteilung neuer Technologien auch die Marktpotenziale hinterfragt würden. Das Unternehmen selbst profitiert von einer Expertise mit hoher Informationsqualität. Es würden nicht nur technologische Aspekte berücksichtigt, sondern auch wichtige Informationen zu Märkten und Wettbewerbern erhoben. In einer solchen Expertise würden zum einen die FuE-Kosten der Erfindung abgeschätzt, und zum anderen die Verwertungs- und Marktchancen beurteilt. Die Kausalkette von der „Invention zur erfolgreichen Innovation“ würde somit um eine Entscheidungshilfe, die Unsicherheiten reduziert, bereichert. Die Kombination der Technologieinformationen der Programme „Feasibility Studies“ bzw. „Start Up- Förderung“ mit den technologiespezifischen Marktinformationen des TecNet verbessern Rentabilitätsrechnungen und helfen bei der Kalkulation des ROI (Return-on-Investment).

\subsection{DAS DEUTSCHE FACHINFORMATIONSZENTRUM KARLSRUHE (FIZ)}

Die Innovationsagentur mbH (Innovationsagentur) wie auch das Fachinformationszentrum Karlsruhe - Gesellschaft für wissenschaftlich-technische Informationen $\mathrm{mbH}$ (FIZ) in Deutschland sind Unternehmen im Bundesbesitz. Das FIZ-Karlsruhe existiert seit 1977, die Innovationsagentur wurde 1984 gegründet. Beide Gesellschaften haben den öffentlichen Auftrag, die heimische Wirtschaft bzw. Wissenschaft durch Serviceleistungen in ihrer Wettbewerbsfähigkeit zu stärken. Zu diesen Serviceleistungen zählt u.a. der Wissens- und Technologietransfer, der in Österreich wie auch in Deutschland in Form von Informationsdienstleistungen angeboten wird. In der Innovationsagentur werden diese Dienstleistungen durch das „TecNet“ bereitgestellt, im deutschen FIZ ist es der „RechercheDienst“. Die Finanzierung dieser Infrastruktur erfolgt in beiden Fällen mittels institutioneller Förderung.

Das TecNet und der RechercheDienst sind zwei Organisationseinheiten staatlicher Informationsinfrastruktur mit einem sich überscheidenden Arbeits- und Aufgabenbereich. Sie bieten sich für einen Vergleich an, weil sie das gemeinsame Ziel verfolgen Technologieunternehmen durch Informationsdienste ein Hilfsmittel zur Umsetzung ihrer Innovationen an die Hand zu geben. Beide Organisationen konzentrieren sich auf einen weit definierten Adressatenkreis, der sich aus Unternehmen, Wissenschaftseinrichtungen und Beratern zusammensetzt. Die Dienstleistung wird in beiden Fällen durch gezielte Informationsrecherchen in Datenbanken erbracht, die z.T. sogar gemeinsam genutzt werden. Beide Einrichtungen halten Vorträge zu ihrem Leistungsangebot und führen Seminare zu Recherchetechniken durch. Die Akquisition, Durchführung und der Abschluss einer Recherche erfolgt durch qualifizierte Ingenieure und Naturwissenschaftler am Sitz der jeweiligen Einrichtung. Die Angebotspalette der verfügbaren Datenbanken, auf die beide Organisationseinheiten zurückgreifen ist weitgehend identisch. Die Rechercheergebnisse werden dem Kunden elektronisch oder in Druckform übermittelt. Sowohl das TecNet als auch der RechercheDienst rechnen ihre Leistungen zu Marktpreisen ab (vgl. Tabelle 18). 
Das FIZ Karlsruhe wurde im Jahr 2000 durch die Unternehmensberatung Arthur D. Little bewertet und neu organisiert. Der Eigenfinanzierungsgrad erreichte inzwischen über 70 Prozent. Aus dem Verkauf von Informationsdienstleistungen wurden rund $€ 20$ Millionen eingenommen; der Umsatz wurde um knapp 15 Prozent gesteigert. Diese Kennzahlen belegen eine erfolgreiche Unternehmensstruktur. Gleichzeitig bietet sich für diese Evaluierung die Chance, die Arbeitsprozesse und Strukturen des RechercheDienstes (FIZ) mit der Struktur des TecNet (Innovationsagentur) $\mathrm{zu}$ vergleichen. Ein solches Benchmarking beinhaltet den Vergleich von Produkten, Dienstleistungen sowie Prozessen und Methoden, um Leistungslücken zu schließen. Die Messlatte in diesem Vergleich ist nicht die Ertragslage bzw. Kostendeckung, sondern die Effizienz interner Arbeitsabläufe.

Tabelle 18: $\quad$ TecNet und FIZ Informationsdienste im Vergleich

\begin{tabular}{|c|c|c|}
\hline & TecNet (Innovationsagentur) & RechercheDienst (FIZ) \\
\hline Organisationsform & $\begin{array}{l}\text { Bereich/Abteilung der } \\
\text { Innovationsagentur (Wien) }\end{array}$ & $\begin{array}{l}\text { Bereich/Abteilung des } \\
\text { Fachinformationszentrums } \\
\text { Karlsruhe }\end{array}$ \\
\hline Mitarbeiter & $\begin{array}{l}1 \text { Person Bereichsleitung } \\
+0,5 \text { Personen Projektassistenz }\end{array}$ & $\begin{array}{l}1 \text { Person Bereichsleitung } \\
\text { (1 StellvertreterIn aus Projektteam) } \\
+11 \text { ProjektmanagerInnen }\end{array}$ \\
\hline Rechercheschwerpunkt & $\begin{array}{l}\text { (Technologieinformationen) }^{*} \\
\text { Marktinformationen }\end{array}$ & Technologieinformationen \\
\hline Angebotspalette & $\begin{array}{l}\text { Ca. } 1.000 \text { Datenbanken, } \\
\text { Marktstudien (auch Auszüge), } \\
\text { Internet-Informationen, } \\
\text { wissenschaftliche Publikationen, } \\
\text { Vermittlung von Experten }\end{array}$ & $\begin{array}{l}220 \text { Online-Datenbanken von STN } \\
\text { International zum Thema } \\
\text { - Wissenschaft und Technik } \\
\text { - Energie- und Patentdatenbanken } \\
\text { Internet-Informationen }\end{array}$ \\
\hline Fachgebiete & $\begin{array}{l}\text { Technologieunspezifisch } \\
\text { (keine Spezialgebiete) }\end{array}$ & $\begin{array}{l}\text { Technologiespezifisch } \\
\text { (Spezialbereiche) }\end{array}$ \\
\hline Recherchegegenstand & $\begin{array}{l}\text { - Marktdaten/Marktanalysen } \\
\text { - Forschungsberichte } \\
\text { - Fachzeitschriften (Aufsätze) } \\
\text { - Studien } \\
\text { - Fachleute/Experten }\end{array}$ & $\begin{array}{l}\text { - Patente/Patentschriften } \\
\text { - „graue Literatur“ } \\
\text { - Forschungsprojekte/-berichte } \\
\text { - Instituts-/Kongressberichte } \\
\text { - Monografien } \\
\text { - Fachzeitschriften (Aufsätze) }\end{array}$ \\
\hline Produkte & $\begin{array}{l}\text { Recherchen (versch. Infoquellen), } \\
\text { Volltexte/Auszüge (Studien etc.) }\end{array}$ & $\begin{array}{l}\text { Recherchen (Datenbanken), } \\
\text { Datenbanken, } \\
\text { Consulting/Schulung, } \\
\text { Vermittlung von Volltexten, } \\
\text { Software etc. }\end{array}$ \\
\hline
\end{tabular}


Tabelle 18 (Fortsetzung): $\quad$ TecNet und FIZ Informationsdienste im Vergleich

\begin{tabular}{lll}
\hline & TecNet (Innovationsagentur) & RechercheDienst (FIZ) \\
\hline Vorteile für Unternehmen & - gezielte Information & - gezielte Information \\
& - aktuelle Marktlage & - aktueller Stand der Technik \\
& - vermeidet Fehlinvestitionen & - vermeiden von Doppelarbeit \\
& - überzeugt Investoren & - vermeidet Fehlinvestitionen \\
& - Zeit- und Geldersparnis & - beschleunigen der Innovations- \\
& - Wettbewerbsvorteile & prozesse \\
& & - Zeit- und Geldersparnis \\
& & - Wettbewerbsvorteile \\
\hline Zielgruppe/Kundenkreis & - Unternehmen & - Unternehmen \\
& junge High-Tech Unternehmen & - Informationsbroker \\
& und Kreditinstitute/Investoren & - Wissenschaft \\
& - Universitäten & - Universitäten \\
& - Wirtschaftsförderer & - Forschungsinstitute \\
& (SeedCapital, BÜRGES) & - Verwaltung/Behörden/Ämter \\
\hline
\end{tabular}

* Das TecNet recherchiert seit 1999 überwiegend technologiespezifische Marktinformationen.

Technologieinformationen, wie z.B. Patentrecherchen fallen nicht in das Angebot des TecNet

Im Folgenden werden die spezifischen Unterschiede des TecNet und des RechercheDienstes herausgearbeitet, mit dem Evaluationsziel, die jeweiligen Stärken bzw. Schwächen der Organisationseinheiten zu identifizieren.

\subsubsection{Organisation}

Das Fachinformationszentrum Karlsruhe (FIZ) wurde als gemeinnützige Gesellschaft mit dem Auftrag gegründet Fachinformationen und darauf basierende Dienstleistungen für Forschung, Entwicklung, Lehre und deren Anwendungsbereiche in Industrie, Wirtschaft und Verwaltung bereitzustellen. Es hat seinen Sitz in Karlsruhe (Deutschland) und beschäftigt rund 330 Mitarbeiter. Ein Drittel dieser Mitarbeiter hat eine wissenschaftliche Ausbildung oder ein Ingenieurstudium. Darüber hinaus haben sich die meisten Beschäftigten EDV- und Dokumentationsfachwissen als Zusatzqualifikation angeeignet. Sie werten internationale Fachpublikationen aus, bringen Informationen strukturiert in Datenbanken ein, entwickeln Recherche-Anwendungssoftware oder schulen ihre Anwender in Seminaren.

Gesellschafter des FIZ sind die Bundesrepublik Deutschland (80 \%), die Bundesländer sowie zahlreiche außeruniversitäre Großforschungseinrichtungen. Das FIZ ist Teil eines Netzwerks der Wissenschaftsgemeinschaft Gottfried Wilhelm Leibniz e.V., einem Zusammenschluss von derzeit 79 wissenschaftlich, rechtlich und wirtschaftlich eigenständigen Forschungsinstituten in Deutschland. Das FIZ gliedert sich in zwei Ergebniscenter (Informationsdienste und Online-Service) sowie zwei Servicecenter (Finanzen/Controlling, Personal, Recht und Infrastruktur). Das Hauptgeschäftsfeld ist der STN-Online-Service, ein internationaler Datenbankzugang zur Nutzung elektronischer 
Datenbanken über wissenschaftliche, technische und ergänzende Wirtschaftsinformationen.

Abbildung 7: „RechercheDienst“ - Organisationseinheit des FIZ Karlsruhe

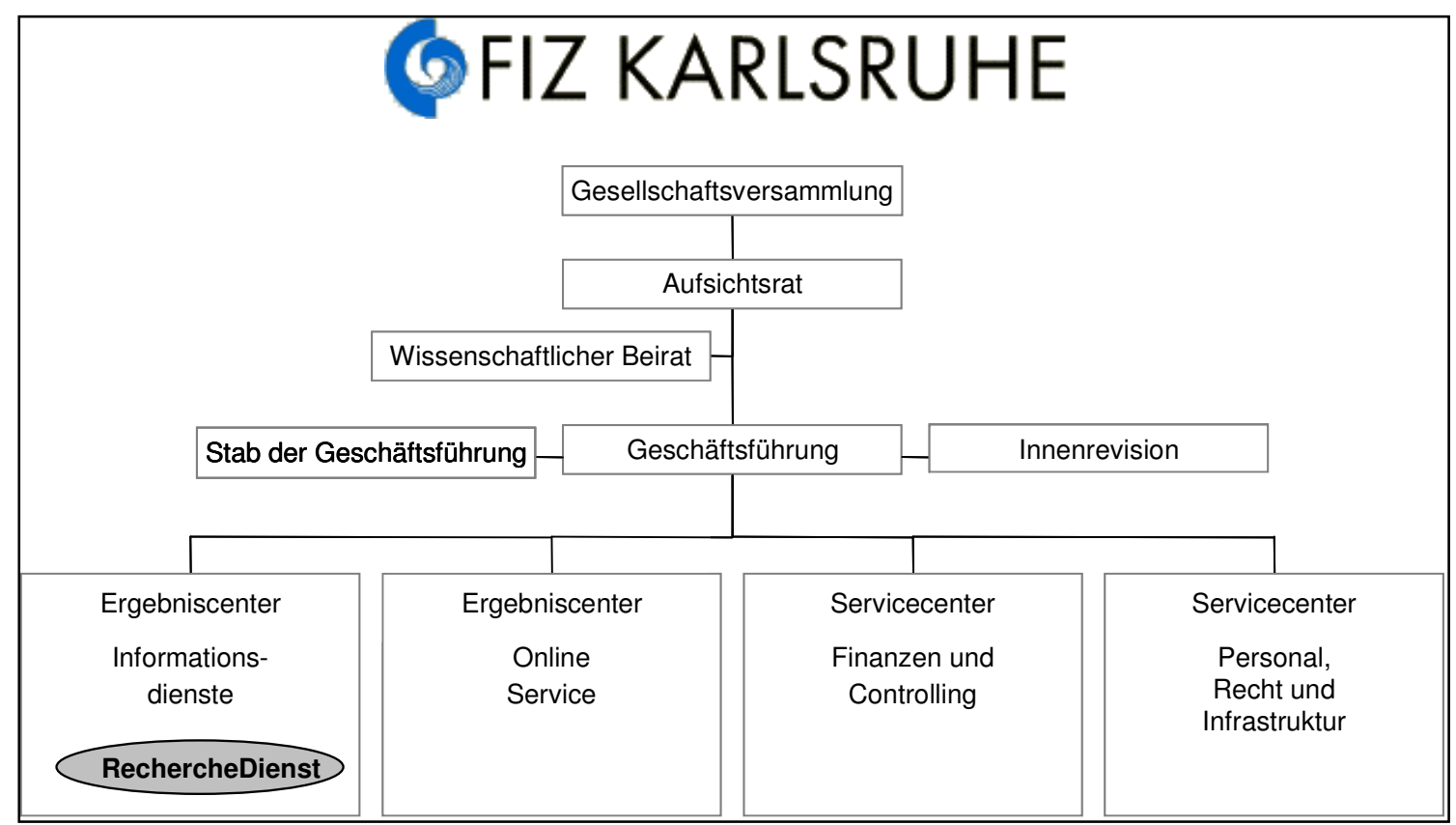

Der „RechercheDienst“ des FIZ ist eine eigenständige Organisationseinheit des Ergebniscenters Informationsdienste und unmittelbar mit dem TecNet vergleichbar. In Deutschland sind in diesem Bereich zwölf hochqualifizierte Mitarbeiter beschäftigt, die auf einzelne Fachgebiete spezialisiert sind. $\mathrm{Zu}$ den insgesamt 14 Fachgebieten zählen beispielsweise die Chemie, die Energie und Umwelt, das Ingenieurwesen, die Luft- und Raumfahrt, Patente und Marken, die Physik etc. Diese Fachgebietsrechercheure, besprechen mit Ihren Kunden die konkrete Anfrage, wählen die relevanten Datenbanken aus und formulieren die Suchstrategie.

\subsubsection{Ziele und Aufgaben}

Unter den Schlagworten „Innovation braucht Information“ versteht sich das FIZ Karlsruhe mit seinem RechercheDienst, ebenso wie das TecNet, als Dienstleister, der weltweit erarbeitetes Wissen so verdichtet und aufbereitet, sodass es selektiv genutzt werden kann und in angemessener Zeit zur Verfügung steht. Die Zielsetzung ist das „Angebot wissenschaftlich-technischer Informationsdienste für Forschung und Lehre, Wissenschaft, Wirtschaft, Technik und Verwaltung“"

Zu den informationspolitischen Geschäftszielen des FIZ zählen:

- Angebot und Förderung der Nutzung elektronischer Fachinformation aus Wissenschaft und Technik und dazugehöriger Wirtschaftsinformation einschließlich der Vermittlung von Volltexten für Forschung, Entwicklung, Lehre und Anwendungsbereiche, auch im Sinne einer Unterstützung und Erweiterung der Tätigkeit wissenschaftlicher Bibliotheken und Verlage 
- Informationsversorgung in Deutschland und damit Beitrag zur Intensivierung des Technologietransfers und der Innovationsförderung

- Informationstransfer ins Ausland zur Darstellung deutscher Forschungs- und Entwicklungsergebnisse sowie die Sicherung des Zugangs zu ausländischer Fachinformation durch bilaterale und internationale Abkommen

- Produktion von Datenbanken

Zu den Aufgabenschwerpunkten zählt:

- Betrieb des Online-Service STN International

- Literaturvermittlung

- Recherchedienst

- Erstellen von Datenbanken und Datenbanksammlungen in elektronischer oder gedruckter Form

- Verlegerdienste

- Internet-Entwicklungen und elektronisches Publizieren

- IT-Dienstleistungen

- Aus- und Fortbildung von Fachleuten für Information und Dokumentation sowie Fachinformatikern

Der RechercheDienst der FIZ bietet eine einmalige oder kontinuierliche Recherche von Fachinformationen zu Wissenschaft und Technik. Die Recherchen erfolgen durch Datenbankabfragen bei STN International und bei Bedarf auch bei anderen Anbietern von Online-Fachinformationen. Diese Fachinformationen werden ergänzt durch Angaben, wie sie auch allgemein im Internet verfügbar sind.

In der Zielsetzung des Dienstleistungsangebots zeigt sich, dass das deutsche FIZ im Unterschied zum TecNet nicht darauf fokussiert ist die Finanzierungsmöglichkeiten von Technologieprojekten zu verbessern. In Deutschland sind die Ziele weiter gefasst. Hier geht es vielmehr um einen nationalen und internationalen Wissens- und Technologietransfer mittels Informationsdienstleistungen. Während das TecNet Finanzierungsentscheidungen bei Kreditinstituten, Förderbanken, VC-Gesellschaften und anderen Kapitalgebern, aber auch bei investitionsbereiten Unternehmen durch Marktinformationen verbessern helfen will, bestehen die Ziele des RechercheDienstes in der Diffusion von Technologieinformationen. In Deutschland sprechen vor allem ordnungspolitische Aspekte dafür, dass ein staatliches Informationsangebot grundsätzlich allen Nachfragern offen steht. Technologietransfer und Innovationen sollen durch Informationsdienstleistungen gefördert werden, u.a. mit dem Ziel die Kommunikation zwischen Wissenschaft und Wirtschaft zu verbessern. Im Unterschied zu diesem unspezifischen Förderansatz orientiert sich das TecNet vorrangig an den Informationsbedürfnissen der Wirtschaft und von Förderbanken. 
Tabelle 19: $\quad$ Auftrag und Ziele des TecNet und des RechercheDienstes

\begin{tabular}{lll}
\hline TecNet (Innovationsagentur) & RechercheDienst (FIZ) \\
\hline - Verbesserung und Verstärkung der & - & Angebot/Förderung/Nutzung elektronischer \\
& Finanzierung von Technologieprojekten & Fachinformationen für Forschung, \\
- & Hilfsmittel für Technologieunternehmen zur & Entwicklung, Lehre und Anwendungsbereiche \\
Umsetzung von Innovationen & - & Informationsversorgung in Deutschland und \\
- & Informationen und Expertengutachten zur & Beitrag zur Intensivierung des Wissens- und \\
Einschätzung des Marktpotenzials von neuen & Technologietransfers \\
Produkten und Technologien & - & Informationstransfer vom/ins Ausland \\
- Chancenausgleich für junge High-Tech & - & Produktion von Datenbanken \\
Unternehmen/Unternehmensgründungen & & \\
\hline
\end{tabular}

Das FIZ ist eine Informationsplattform, das seinen Kunden eine systematische Informationsinfrastruktur zur Verfügung stellt. Der RechercheDienst recherchiert, weitgehend unabhängig von der Zielsetzung und Strategie des Kunden, nach einzelnen Begriffen, Volltexten oder Patentschriften. Das FIZ eröffnet mit seinen Diensten lediglich den Zugang zu Informationen, die Hintergründe, der Zweck, der Nutzen und die Verwendung der Informationen bleiben jedoch weitgehend unbekannt.

Das TecNet recherchiert demgegenüber stärker kundenorientiert. Es berücksichtigt die Ziele seiner Kunden und recherchiert in Bezug auf diese Ziele: Marktinformationen für einen Business-Plan mit dem Ziel Kapitalgeber zu überzeugen, Marktinformationen für etablierte Unternehmen mit dem Ziel Marktpotenziale zu erkunden, Marktinformationen für Investoren um das Risiko einer Investition zu kalkulieren.

\subsubsection{Zielgruppe}

Die Kunden des RechercheDienstes kommen aus allen Bereichen der Wirtschaft, aus der Verwaltung, den Hochschulen und Forschungseinrichtungen. Hierzu zählen die klassischen Informations- und Dokumentationsabteilungen der Großindustrie, Anwender in kleinen und mittleren Unternehmen bis hin zu selbständigen Beratern. Sämtliche Informationsdienstleistungen stehen auch allen interessierten Bürgern und Bürgerinnen zur Verfügung.

Das FIZ-Karlsruhe kooperiert mit Chemical Abstracts Service in Ohio (USA) und der Japan Science and Technology Cooperation (JST) in Tokio. Gemeinsam wird das „Scientific and Tecnical Information Network“ (STN International) betrieben. STNInternational ist ein Online- Informationsnetzwerk für öffentlich zugängliche Datenbanken aus/für Wissenschaft und Technik. Als STN Servicezentrum Europa ist das FIZ insbesondere für die Geschäftsabwicklung in Europa verantwortlich. 


\subsubsection{Produkte}

STN International und der RechercheDienst decken mit ihrem Angebot alle naturwissenschaftlichen und technischen Fachgebiete ab. In den Datenbanken finden sich Literaturhinweise, Volltexte, numerische und Faktendaten sowie Patentinformationen und ergänzende Wirtschaftsinformationen zu:

- Agrar- und Ernährungswissenschaften

- Astronomie und Astrophysik

- Biowissenschaften und Biotechnologie

- Chemie und verwandte Gebiete

- Computertechnologien

- Energieforschung und -technologie

- Elektrotechnik und Telekommunikation

- Informatik und Informationswissenschaften

- Ingenieurwissenschaften und Bauwesen

- Kernforschung und Kerntechnik

- Konstruktion

- Luft- und Raumfahrt

- Materialwissenschaften

- Mathematik

- Medizin

- Metallurgie

- Patentinformationen

- Pharmazie

- Physik

- Umweltforschung und -technologie.

Der RechercheDienst hilft Unternehmen oder Erfindern beispielsweise beim gewerblichen Schutz einer Erfindung, indem eine Patentrecherche feststellt, ob eine Erfindung überhaupt patentwürdig ist.

Ein wesentliches Merkmal in dem sich das TecNet vom RechercheDienst unterscheidet, liegt im Rechercheschwerpunkt. Während der RechercheDienst reine ,Technologieinformationen anbietet“, hat sich das TecNet auf ,technologierelevante Marktinformationen“ spezialisiert. Dieser semantische Unterschied ist bedeutend, weil das TecNet Informationen über Technologien und Märkte recherchiert, während der RechercheDienst konstruktionsrelevante Technologieinformationen aufbereitet.

\subsubsection{Dienstleistung und Finanzierung}

Der Kunde des FIZ-Karlsruhe kann auf mehreren Wegen zu den von ihm gesuchten Informationen gelangen. Zum einen kann er als Gast in kostenlosen Datenbanken recherchieren bzw. eine Recherche (Datenbankabfrage) üben. Zweitens kann er als registrierter Nutzer in kostenpflichtigen Datenbanken selbständig recherchieren. Drittens kann er den RechercheDienst des FIZ in Anspruch nehmen und Informationen kostenpflichtig durch Fachreferenten recherchieren lassen. 


\section{Online-Recherche}

Entscheidet sich ein Kunde zur Eigenrecherche, dann sind zunächst juristische Formalitäten in Form eines Online-Nutzungsvertrags zu lösen. Dieser Vertrag enthält Bestimmungen zu den Rechten und Pflichten des Kunden bei Zugriff auf die FIZDatenbanken, regelt das Copyright usw. Für die Nutzung wird eine Jahresgebühr in Höhe von $€ 30,00$ erhoben. Jede Recherche in einer Fachdatenbanken (Patent-, Bio-, Chemiedatenbanken etc.) wird nach einer „Anschaltzeit" abgerechnet. Diese Zeit (Stunden/Minuten) gibt an, wie teuer es für den Kunden ist, auf eine Spezialdatenbank zuzugreifen. Die Kosten liegen bei $€$ 0,28 bis zu $€$ 6,25 die Minute. Darüber hinaus wird die Ausgabe der Information als „Print“ oder als „Display“ separat berechnet. Gedruckte Ausgaben (Print) sind i.d.R. geringfügig teurer als reine Bildschirmausgaben (Display). Hier liegen die zusätzlichen Kosten bei $€ 0,20$ und über $€ 40,00$. Darüber hinaus kann man kostenpflichtige „Suchläufe“ bzw. „Frequenzsuchen“ (täglich/wöchentlich/monatlich) in Auftrag geben. Die Recherchekosten berechnen sich wie folgt:

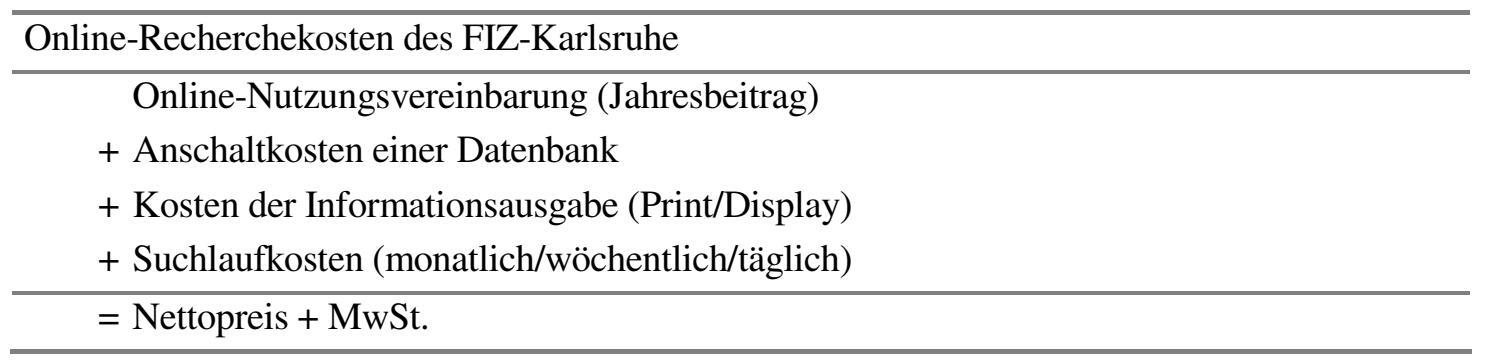

\section{RechercheDienst}

Kunden, die in der Abfrage von Datenbanken wenig versiert sind, oder die keinen OnlineNutzungsvertrag abschließen wollen, greifen bei der Informationssuche auf den RechercheDienst zurück. Das Dienstleistungsangebot des RechercheDienstes setzt sich aus vier Produkten zusammen. Zu diesen zählen (a) die fachliche Auskunft/Kurzauskunft, (b) die Standardrecherche, (c) die Spezialrecherche sowie eine (d) Abo-Recherche.

Für eine einfache fachliche Auskunft (Kurzrecherche) fallen beim RechercheDienst des FIZ $€ 55,00$ als Grundpauschale an. Eine Standardrecherche berücksichtigt mehrere Kriterien und kostet $€ 120,00$. Eine Spezialrecherche schließt eine Vorabinformation des Kunden über erste Rechercheergebnisse mit weiteren Selektions- und/oder Änderungsmöglichkeiten ein und kostet Pauschal $€ 280,00$. Alle Beträge erhöhen sich um die Kosten für die Informationsausgabe (Display oder Print) und sind Nettokosten. Abgerundet wird das Informationsangebot mit einer AboRecherche, die im Anschluss an die Ausführung einer Recherche erfolgt. Nach Abstimmung mit dem Kunden wird die Suchstrategie/formulierung festgelegt, ebenso die Suchfrequenz (wöchentlich/14-tägig/monatlich) und die "Strategieüberprüfungstermine" (stimmt die Suchformulierung noch mit dem benötigten Informationsbedarf überein?). Für die AboRecherche ist der Abschluss einer Online-Nutzungsvereinbarung zwingend. Mindestlaufzeit einer AboRecherche ist ein Jahr mit einer Kündigungsfrist von drei Monaten.

Grundsätzlich setzt sich ein Rechercheprojekt aus folgenden Kosten zusammen:

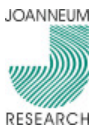




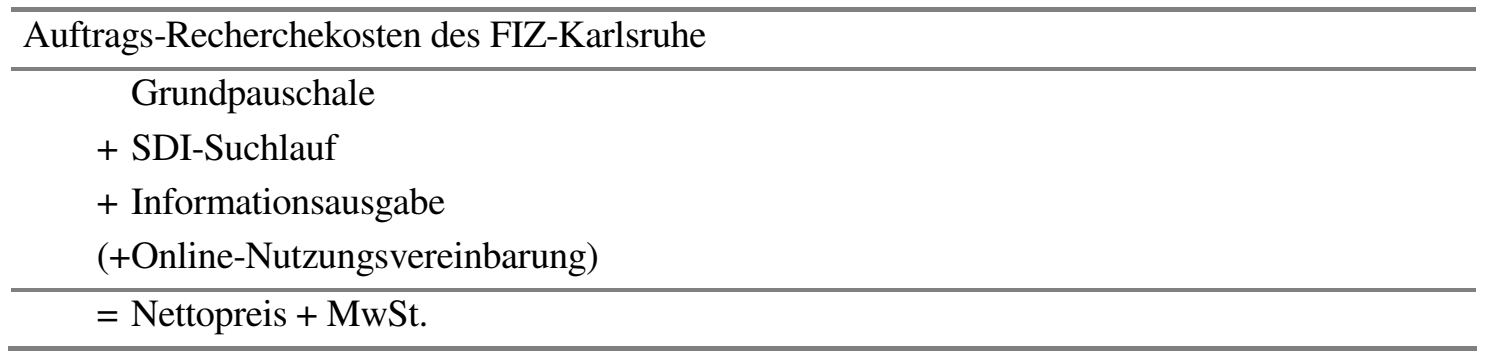

Da das FIZ-Karlsruhe im öffentlichen Auftrag tätig ist, werden dem akademischen Bereich erhebliche Rabatte (bis zu $90 \%$ ) und Festpreise eingeräumt.

Das TecNet unterscheidet sich vom RechercheDienst durch ein anderes Verrechnungssystem. Beide Gesellschaften verlangen eine Grundpauschale (Fixkosten) sowie aufwandsbezogene Kosten (variable Kosten). Im Vergleich der beiden Informationsanbieter wird bei den variablen Kosten im TecNet jedoch der Personalaufwand berechnet, während der RechercheDienst überwiegend die „Datenbankanfragen“ (Zugriffszeit) abrechnet.

Tabelle 20: $\quad$ Kosten der Informationsrecherche im Vergleich

\begin{tabular}{|c|c|c|}
\hline & TecNet (Innovationsagentur) & RechercheDienst (FIZ) \\
\hline „Online“-Nutzungsvereinbarung & - nicht vorhanden - & $30,00 €$ (Jahresbeitrag) zzgl. MwSt. \\
\hline $\begin{array}{l}\text { Kurzrecherche/fachliche } \\
\text { Auskünfte }\end{array}$ & $\begin{array}{l}\text { kostenlos im Rahmen der } \\
\text { Vorrecherche }\end{array}$ & $\begin{array}{l}\text { 55,00€ (Pauschale) zzgl. MwSt. } \\
\text { + Online-Kosten }\end{array}$ \\
\hline Standardrecherche & \multirow{2}{*}{$\begin{array}{l}€ 181,68 \text { (Pauschale für externe } \\
\text { Kunden) + € 90,84 Stundenlohn } \\
\text { + variabler Anteil für Studien etc. } \\
\text { (alle Preise inkl. MwSt.) }\end{array}$} & $\begin{array}{l}\text { 120,00 } €(\text { Pauschale }) \text { zzgl. MwSt. } \\
\text { + Online-Kosten }\end{array}$ \\
\hline Spezialrecherche & & $\begin{array}{l}\text { 280,00 €(Pauschale) zzgl. MwSt. } \\
\text { + Online-Kosten }\end{array}$ \\
\hline $\begin{array}{l}\text { AbonnementRecherchen } \\
\text { (AboRecherche) }\end{array}$ & - nicht vorhanden - & $\begin{array}{l}\text {... wie Standard-/Spezialrecherche } \\
\text { Abo-Pflege: nach Aufwand }\end{array}$ \\
\hline
\end{tabular}

Das TecNet recherchiert nach Arbeitsaufwand (Stundensätze), während der RechercheDienst jede einzelne Abfrage (Zugriffszeit) in Rechnung stellt. Ein Suchlauf wird dem Kunden beim RechercheDienst mit einem eigenen Preis berechnet.

Der RechercheDienst bevorzugt es, seine Datenbanken als eigenständige Produkte anzubieten. Jede einzelne Datenbank wird mit ihren Preisen (Anschaltzeit, Display, Print, Suchlauf, Frequenzlauf, MwSt.) in einer Liste geführt und ist im Internet abrufbar. Das TecNet erstellt dagegen individuelle Angebote und publiziert keine Preise im Internet oder auf seinen Informationsfoldern. Die Kostenstruktur ist hier weniger transparent. Während sich die Kosten beim RechercheDienst für interessierte Kunden unmittelbar abschätzen lassen, sind die Kosten für Informationsrecherchen im TecNet zunächst nicht kalkulierbar.

Ein für die Kostendeckung bedeutender Unterschied zwischen TecNet und dem RechercheDienst ergibt sich aus dem Verhältnis des individuellen Arbeitsaufwands zur Abrechnung: Während beim TecNet das Prozedere „Anfrage-VorrechercheAbstimmung-Auftrag-Suchstrategie-Recherche-(Zwischenbericht-Recherche)-Endber- 
icht“ abläuft, beschränkt sich der RechercheDienst auf „Anfrage-Auftrag-SuchstrategieRecherche-Output"“.

Die Abstimmung und Definition der Suchstrategie ist beim RechercheDienst der wesentliche Arbeitsaufwand des Rechercheurs. Die eigentliche Recherche und den Output übernimmt der Computer. Im TecNet zieht sich der Arbeitsaufwand von Beginn der Anfrage bis zur Erstellung eines individuellen Endberichts durch. Die Recherche wird hier nicht „mechanisch“ der EDV überlassen. Die Datenbank- oder Studienergebnisse werden im TecNet gesichtet, beurteilt und in den Kontext der Ziele bzw. des Auftrags des Kunden gestellt.

\subsubsection{Auftragsabwicklung}

$\mathrm{Zu}$ den wichtigsten Serviceleistungen des FIZ zählen Datenbankzugänge, die ein Kunde „online“ abrufen kann. Das FIZ stellt eine elektronische Plattform mit z.T. selbstentwickelter Recherchesoftware zur Verfügung, die eine komfortable Informationssuche ermöglicht. Darüber hinaus werden Anwender durch die Vermittlerdienstleistungen des RechercheDienstes betreut und Recherchen als kostenpflichtige Serviceleistungen angeboten. Die Auftragsabwicklung im FIZ lässt sich wie folgt beschreiben (vgl. Abbildung 8):

1. Kundenanfrage (telefonisch, Email oder persönlich)

2. Der Kunde hat die Wahl, ob er

... selbständig ,online“ recherchieren möchte (Nutzungsvertrag)

... den Recherchedienst des FIZ beauftragt

3. Durchführung der Recherche („online“ oder RechercheDienst)

4. Output ("Display" oder "Print")

5. Erfolgsunabhängige Rechnungserstellung

Das FIZ bietet seinen Kunden

- den Online-Zugang zu den bei den STN-Servicezentren aufliegenden Datenbanken der verschiedenen Datenbankhersteller,

- einen RechercheDienst, sowie

- die Online-Bestellung von Dienstleistungen Dritter (z.B. Online-Ordering von Volltexten) an.

Das Produkt „FIZ AutoDoc“ ist z.B. ein automatischer Volltextvermittlungs-Service mit dem sich elektronische und gedruckte Dokumente über Internet beschaffen lassen. Mit diesem Vermittlungsdienst wird ein zentraler Service für den Kunden auf der einen Seite und ein Abrechnungsservice für die Volltextlieferanten auf der anderen Seite angeboten. Zeitschriftenartikel und Patente werden automatisch vermittelt, wobei die Daten aus STNDatenbanken direkt ins System übernommen werden können. Studien, Bücher, Forschungsberichte usw. können über „FIZ AutoDoc“ direkt zum Kauf bestellt werden. 


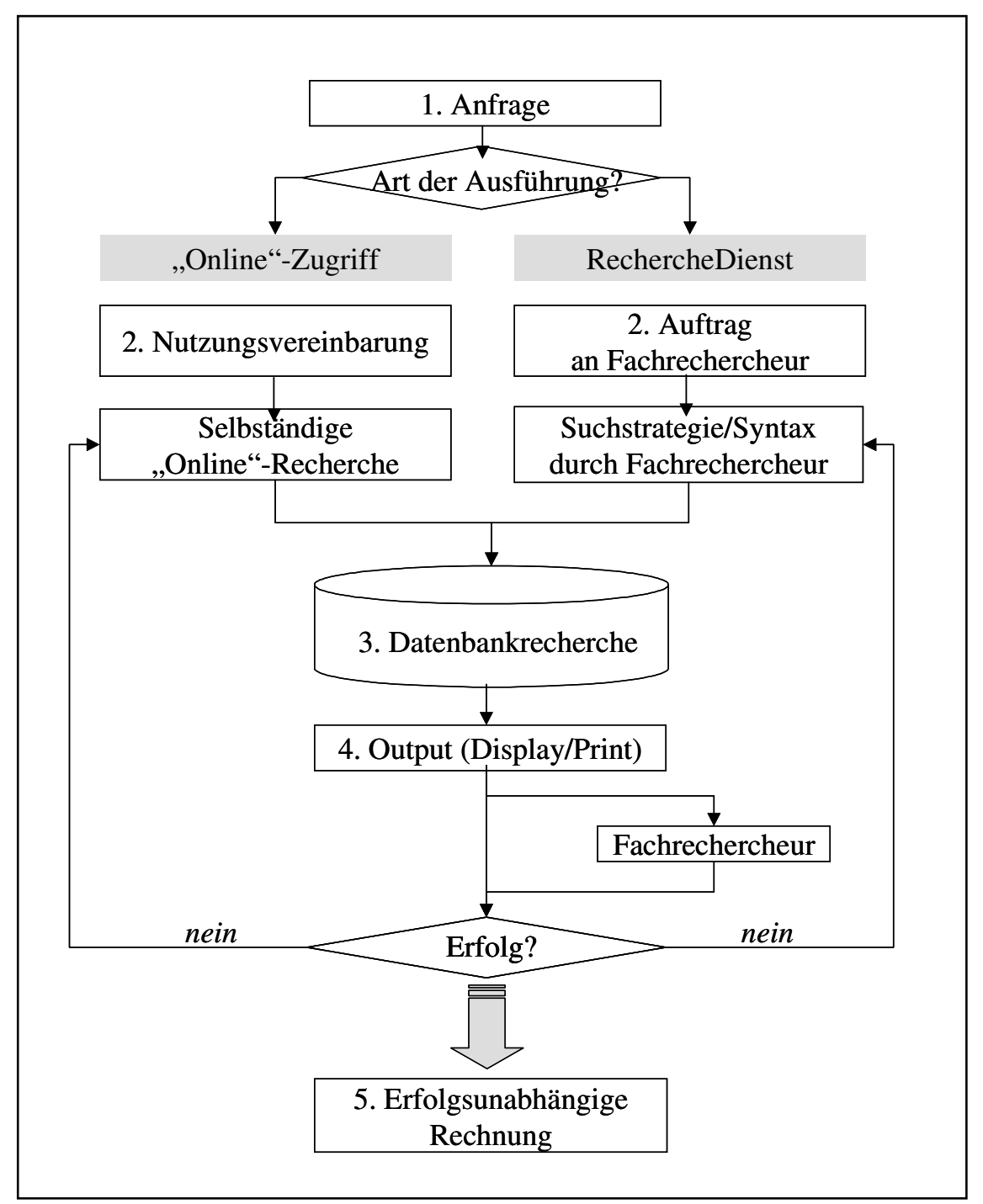

Das Angebot des TecNet ist kundenspezifischer, aber auch aufwendiger. Während beim RechercheDienst häufig eine gezielte kostenpflichtige Datenbankabfrage durchgeführt wird, sammelt das TecNet Informationen aus mehreren Datenbanken und Informationsquellen (Studien), verdichtet diese und erstellt zusätzlich einen kompletten Bericht. Im Unterschied $\mathrm{zu}$ dieser umfassenden Serviceleistung wird am FIZ lediglich eine automatische Datenbankrecherche gestartet und das unmittelbare Datenbankergebnis angezeigt bzw. ausgedruckt.

Nimmt der Kunde den RechercheDienst in Anspruch, so werden die Online-Abfragen durch die Experten am FIZ in Karlsruhe ausgeführt.

Während das TecNet mehrere Informationsquellen berücksichtigt, bemüht sich der Fachrechercheur des RechercheDienstes durch eine einzige Abfrage das gewünschte Resultat zu erzielen. Ein Kunde des FIZ kann auf die Datenbanken auch ohne den RechercheDienstes zugreifen und in verschiedenen Datenbanken nach den gewünschten Informationen suchen. Da allerdings jede Abfrage in den Datenbanken des FIZ kosten- 
pflichtig ist, profitiert der Kunde von einem Rechercheexperten (RechercheDienst), der, wie ein Bibliothekar, über das Know-how verfügt in welcher der zahlreichen Datenbanken die Informationen verfügbar sind. Der Fachrechercheur hat die Qualität und Strukturen seiner Fachdatenbanken als Wissensschatz im Kopf und ruft Informationen gezielt ab. Im Allgemeinen kann er Fachinformationen abrufen ohne über mehrere Datenbanken zu ,surfen“. Die eigentliche Recherchetätigkeit beschränkt sich (weitgehend) auf den Abfragesyntax.

Das TecNet dagegen ,screent“ mehrere Datenbestände und recherchiert in verschiedenen Informationsquellen. Hier beschränkt sich die Recherche nicht auf den Abfragesyntax, sondern es wird im Kontext recherchiert. Das TecNet verschafft sich durch eine Vorrecherche zunächst einen Überblick über das Aktionsfeld des Kunden und den dort verfügbaren Informationsquellen. Bei einer ausreichenden Informationslage, wird dem Kunden ein Angebot unterbreitet und die Recherche durchgeführt. Dieses Verfahren ist im TecNet erforderlich, weil

- Märkte komplexer sind als spezifische Technologien (Komplexität);

- Fachrecherchen nicht auf mehrere Personen verteilt sind (Arbeitsteilung problematisch);

- Märkte im Unterschied zu Technologien kaum klassifiziert sind (Systematisierung);

- Rechercheergebnisse nicht nur kundengerecht aufbereitet, sondern an den Zielen des Kunden ausgerichtet werden (Kontext).

Der Rechercheprozess im TecNet ist aufwendiger, weil einzelne Internet-Seiten bzw. Datenbanken in der Vor- und Hauptrecherche wiederholt abgefragt werden. Hier entsteht Redundanz. Ferner recherchiert das TecNet so lange, bis die vereinbarte Informationsdienstleistung von (subjektiv) angemessener Qualität und Güte ist. Die Ansprüche an die eigenen Leistungen sind dabei hoch - und der Rechercheprozess umso aufwendiger.

\subsubsection{Fazit}

Das österreichische TecNet und der deutsche RechercheDienst sind zwei eigenständige Organisationseinheiten staatlicher Informationsinfrastruktur. Beide Dienste recherchieren wissenschafts- und unternehmensrelevante Informationen in Datenbanken mit dem Ziel, Prozesse der Wissens- und Entscheidungsfindung zu verbessern. Der staatliche Auftrag an das TecNet lautet, durch ein Informationsangebot die Finanzierungsmöglichkeiten von Technologieprojekten zu verbessern sowie Technologieunternehmen bei der Umsetzung von Innovationen behilflich zu sein. Der RechercheDienst verfolgt ganz allgemein das staatliche Ziel, elektronische Fachinformationen für eine bessere Informationsversorgung in Deutschland bereitzustellen.

Das TecNet recherchiert „technologierelevante Marktinformationen“ ohne sich auf eine spezifische Technologie zu spezialisieren. Dagegen konzentriert der RechercheDienst seine Informationsrecherchen auf bestimmte naturwissenschaftliche und technische Fachgebiete. Dieser Fokus spiegelt sich auch in der Organisations-, Ablauf- und 
Preisstruktur des RechercheDienstes wieder und gewährleistet ein differenziertes Angebot.

Ausgangspunkt des deutschen Dienstleistungsangebots ist das Hauptprodukt des FIZ Karlsruhe: umfangreiche, internationale Technologiedatenbanken. Auf diesen Produkten aufbauend, hat sich der RechercheDienst als Unterstützungsservice für informationssuchende Kunden etabliert. Qualifizierte Experten sind beim RechercheDienst für die Recherche in technologiespezifischen Fachdatenbanken verantwortlich. Aufgrund identischer Abfragestrategien und angesichts einer fachübergreifenden Zusammenarbeit, können sich die Rechercheure im Bedarfsfall gegenseitig vertreten und auch Fachvertreter aus anderen Abteilungen des FIZ hinzuziehen. Der Kunde zahlt grundsätzlich für das Hauptprodukt: den Datenbankzugriff, die enthaltene Information und den Datenbankoutput. Er hat allerdings die Wahl, ob er Datenbankzugriffe selbständig (Online-Service) ausführt, oder durch die Experten des RechercheDienstes ausführen lässt. Die Dienstleistung des RechercheDienstes wird dann gesondert in Rechnung gestellt. Diese systematische Ablauforganisation wird von einem detaillierten Preissystem flankiert, das Interessenten und Kunden die Informationskosten transparent macht. Weil das Betreiben komplexer Datenbanksysteme wegen fortlaufend zu aktualisierender Inhalte und der erforderlichen technischen Pflege/Wertung sehr kostenintensiv ist, wird vor allem die Informationsqualität und -bereitstellung mit einem Preis bewertet. Das FIZ arbeitet mit dieser Preisphilosophie effizient, weil der Rechercheaufwand in einzelne Kostenpositionen gesplittet wird. Die Recherchedienstleistung und der Wert der Information werden separat abgerechnet.

Das TecNet vermarktet gegenüber dem RechercheDienst nicht seine Datenbanken sondern seine Recherchedienstleistung als Hauptprodukt. Die Informationsleistung wird marktspezifisch und kundenorientiert erbracht. Sie ist nicht auf eine Spezialdatenbank beschränkt sondern erfordert eine Sichtung mehrerer Informationsquellen. Ein Arbeitsteilung ist aufgrund der Personalstärke nicht möglich. Die Informationsrecherche ist vergleichsweise weniger formalisiert und systematisiert. Darüber hinaus wird nicht nur ein Schlüsselbegriff sondern im Zielkontext des Kunden recherchiert. Während der Recherchegegenstand beim deutschen RechercheDienst sehr spezifisch vorgegeben ist, muss sich das TecNet zunächst die relevante Technologie studieren und sich dann eine zugehörige Markttransparenz verschaffen. Die Marktrecherche unterscheidet sich von der reinen Technologierecherche damit durch höhere Komplexität und Aufwand. Sie ist vor allem weniger spezifisch, so dass sich der „Suchaufwand“, das Eingrenzen des eigentlichen Recherchegegenstandes als schwierig gestaltet. Hier überwiegen die Recherchekosten (Zeitbedarf) die Informationskosten (Datenbankbereitstellung und -output).

Das TecNet unterscheidet bei der Preissetzung zwar zwischen externen Kosten z.B. für Studien, nicht aber zwischen Recherchekosten und Informationskosten. Diese, beim RechercheDienst gesplitteten Positionen, werden beim TecNet pauschal in Form einer Recherchepauschale und einem Stundensatz abgegolten. Die Kostenstruktur belastend kommt hinzu, dass vor einer Auftragsvergaben Vorrecherchen durchgeführt werden. Da das TecNet diese aufwendigen Vorrecherchen nicht kalkuliert, Informationskosten 
(Datenbankzugriffe) nicht separat berechnet und vor der Auftragsvergabe Informationsquellen preisgibt, ist der Deckungsbeitrag gering. Zudem werden einzelne Arbeitsschritte nach einer Auftragsvergabe wiederholt, so dass es zu Redundanz kommt. Auch wenn man argumentiert, dass somit die Kosten der Vorrecherche im eigentlichen Projekt berücksichtigt werden, ist dieser Gedankengang nicht stichhaltig: Da der eigentliche Aufwand in der Suche der Informationen liegt, die nach erfolgreicher Vorrecherche in Teilbereichen abgeschlossen ist, werden in der Hauptrecherche nur noch die erfolgreichen Suchschritte wiederholt und berechnet. Die nicht erfolgreichen Rechercheschritte sind für das TecNet ,sunk costs“, die zwar entstanden, nicht aber auf den Kunden abgewälzt werden. Hier besteht ein wesentlicher Unterschied zu anderen Informationsbrokern wie z.B. dem RechercheDienst. Das Preisgefüge ist hier unmittelbar an einen Auftrag geknüpft. Jede Datenbankabfrage hat ihren Preis, auch wenn sie Informationen liefert, die der Kunde nicht für seine Ziele verwenden kann. Unsicherheiten über den Rechercheerfolg werden nicht durch eine Vorrecherche aufgefangen. In diesem Punkt wäre vom TecNet nach einer zweijährigen Erprobungsphase und mit der aufgebauten Erfahrung zu wünschen, dass es Rechercheaufträge in ihren Erfolgsaussichten zukünftig auch ohne Vorrecherche einschätzen kann. 


\section{Entwicklungsperspektiven}

In der vorliegenden Evaluation wurden zunächst diejenigen Objekte (Ziele, Produkte, Methoden, Prozesse etc.) ausgewählt die im Auftrag des BMWA analysiert, begutachtet und verglichen werden sollten. In einem zweiten Schritt erfolgte die Datenerhebung (Primärinformationen) in Form einer Sichtung der Vertragsgrundlagen, einer schriftlichen Befragung sowie in Form mehrerer Expertengespräche. Im dritten Evaluationsschritt wurden die Entwicklung sowie die Potenziale der wichtigsten Zielgruppe des TecNet analysiert. Eine anschließende Unternehmensbefragung von TecNet-Kunden, Technologieunternehmen, Wissenschaftseinrichtungen und Förderinstitutionen gab Aufschluss über die Zufriedenheit, Wahrnehmung und Leistungsmerkmale von TecNet. Den Abschluss bildete ein programmatischer sowie ein institutioneller Vergleich. Dabei war es wichtig festzulegen, welche Ähnlichkeiten zur Gewährungsleistung der Vergleichbarkeit gegeben sein sollten. Anhand der Vertragsbedingungen, Befunde und Leistungsindikatoren konnte die Zielerreichung und Effizienz beurteilt werden, die sich als Ergebnis in den einzelnen Fazits und der Zusammenfassung wiederfinden.

An dieser Stelle werden - quasi als Synthese der Ergebnisse - Entwicklungsperspektiven aufgezeigt, die als Diskussionsgrundlage und Anregung dienen sollen, um die Stärken des TecNet weiter auszubauen bzw. um Leistungslücken zu schließen. Die folgenden Szenarien wurden unter der Prämisse entwickelt, dass der Entwurf zum „Austria Wirtschaftsförderung - Gesetz“, der gerade politisch debattiert wird, zumindest in groben Zügen auch verabschiedet wird.

\section{Szenario: TecNet als Querschnittsbereich}

- TecNet wird als interne Dienstleistung für andere Bereiche der Austria Wirtschaftsservice GmbH (in der ja die Innovationsagentur, FGG, Bürges, ERP aufgehen wird) definiert.

- Akquisition externer Aufträge fällt aus; Konzentration auf interne Projekte

- TecNet positioniert sich als ,interne Servicestelle“

- Klar definiertes Angebot für die Gruppe FGG, Bürges, ERP, für Patentverwertung und für den Bereich „Start-Up“ ist zu entwickeln.

- Transparente Leistungsverrechnung muss Bedingung sein.

\section{Szenario: TecNet fokussiert}

- TecNet wird auf „Start-Ups” und „Start-Up“-Geldgeber fokussiert.

- Organisatorisch wird TecNet Bestandteil des Start-Up Bereichs der Austria Wirtschaftsservice $\mathrm{GmbH}$

- Kundengruppen eines fokussierten TecNet sind weiterhin interne Organisationseinheiten der Innovationsagentur (z.B. SeedCapital, TecMa), extern aber in erster Linie ErfinderInnen und GründerInnen. 
- Marketingaktivitäten werden auf GründerInnen abgestimmt (Zusammenarbeit mit dem Gründerservice von Banken, Ausbau der Zusammenarbeit mit AplusB)

- Entwicklung von Leistungsbildern: Business-Plan-Unterstützung, Marktchancenabschätzung u.ä.

\section{Szenario: TecNet startet durch}

- TecNet wird personell und finanziell so ausgestattet, das mit der bestehenden Ziel- und Zielgruppendefinition ein vernünftiges Arbeiten möglich ist (Arbeitsteilung, Organisation)

- Es kommt zu einer Produkt- und Preisdifferenzierung

- TecNet ist bestrebt, in der Zusammenarbeit mit Unternehmen (zumindest) marktnahe Preise zu setzen.

- Die Gruppe GründerInnen und ErfinderInnen wird weiterhin durch öffentliche Mittel unterstützt.

- TecNet baut den Ruf des High-Tech Spezialisten weiter aus. 
Materialienanhang zum

Abschlussbericht

\section{Evaluierung von TecNet - Netzwerk für Markt- und Technologieinformationen der Innovationsagentur}

Wien, 31.Juli 2002

Ansprechpartner:

Dr. Andreas Fier/ Mag. Klaus Zinöcker Institut für Technologie- und Regionalpolitik

Joanneum Research Forschungsgesellschaft mbH Wiedner Hauptstr. 76

A-1040 Wien

Telefon: +43 (1)581 7520 - 2811

Fax: $\quad+43$ (1)581 $7520-2820$

Email: andreas.fier@joanneum.at

klaus.zinöcker@joanneum.at 


\section{MATERIALIENVERZEICHNIS}

Materialien 1: Angebot: Aufbau einer Patentverwertungsagentur und eines Netzwerkes für Technologiebewertung (10. Juli 1997)

Materialien 2: Vertrag: Aufbau einer Patentverwertungsagentur und eines Netzwerkes für Technologiebewertung (10. Juli 1997)

Materialien 3: Angebot: Weiterführung und Ausbau von TecNet - Das Netzwerk für Markt und Technologieinformationen (29. Mai 2001)

Materialien 4: Vertrag: TecNet - Das Netzwerk für Markt und Technologieinformationen (29. Mai 2001)

Materialien 5: Evaluationsunterlagen: Schriftliche Befragung des TecNet

Materialien 6: Anschreiben: TecNet Evaluation - Umfrage bei Unternehmen und Wissenschaftseinrichtungen

Materialien 7: Fax-Fragebogen: Nicht-TecNet-Kunden

Materialien 8: Anschreiben: TecNet Evaluation - Umfrage bei TecNet-Kunden

Materialien 9: $\quad$ Fax-Fragebogen: TecNet-Kunden

Materialien 10: Anschreiben: TecNet Evaluation - Umfrage bei Förderinstitutionen

Materialien 11: Fax-Fragebogen: Förderinstitutionen

Materialien 12: Jahresbericht: Jahresbericht des TecNet 2000

Materialien 13: Jahresbericht: Jahresbericht des TecNet 2001

Materialien 14: Projekte: Auftragnehmer und Erträge

Materialien 15: Anschreiben: Kundenanschreiben des TecNet

Materialien 16: Recherchebericht: Muster eines Rechercheberichts

Materialien 17: Rechercheabrechung: Muster einer Rechercheabrechnung

Materialien 18: Werbefolder: Wissen_ist_Markt

Materialien 19: Werbeinformationen: Werbeinformationen des TecNet

Materialien 20: Success-Stories: Erfolgsbeispiele

Materialien 21: Vorträge: Vortragsfolien/Präsentationen

Materialien 22: Studie: KPMG - Wirtschaftpolitische Aktivitäten in Europäischen Regionen

Materialien 23: Informationsquellen: Informationsquellen \& Datenbanken

Materialien 24: Informationsquellen: Die fünf bedeutendsten Datenbanken des TecNet

Materialien 25: Expertengespräche: Evaluation und Expertengespräche

Materialien 26: Förderlinien: Adressen und Ansprechpartner von Förderinstitutionen 
Materialien 1: Angebot: Aufbau einer Patentverwertungsagentur und eines Netzwerkes für Technologiebewertung (10. Juli 1997)

Materialien 2: Vertrag: Aufbau einer Patentverwertungsagentur und eines Netzwerkes für Technologiebewertung (10. Juli 1997)

Materialien 3: $\quad$ Angebot: Weiterführung und Ausbau von TecNet-Das Netzwerk für Markt und Technologieinformationen (29. Mai 2001)

Materialien 4: Vertrag: TecNet-Das Netzwerk für Markt und Technologieinformationen (29. Mai 2001)

Materialien 5: $\quad$ Evaluationsunterlagen: Schriftliche Befragung des TecNet

Materialien 6: $\quad$ Anschreiben: TecNet Evaluation-Umfrage bei Unternehmen und Wissenschaftseinrichtungen

Materialien 7: Fax-Fragebogen: Nicht-TecNet-Kunden

Materialien 8: $\quad$ Anschreiben: TecNet Evaluation - Umfrage bei TecNet-Kunden

Materialien 9: Fax-Fragebogen: TecNet-Kunden

Materialien 10: Anschreiben: TecNet Evaluation-Umfrage bei Förderinstitutionen

Materialien 11: Fax-Fragebogen: Förderinstitutionen

Materialien 12: Jahresbericht: Jahresbericht des TecNet 2000

Materialien 13: Jahresbericht: Jahresbericht des TecNet 2001

Materialien 14: Projekte: Auftragnehmer und Erträge

Materialien 15: Anschreiben: Kundenanschreiben des TecNet

Materialien 16: Recherchebericht: Muster eines Rechercheberichts

Materialien 17: Rechercheabrechung: Muster einer Rechercheabrechnung

Materialien 18: Werbefolder: Wissen_ist_Markt

Materialien 19: Werbeinformationen: Werbeinformationen des TecNet

Materialien 20: $\quad$ Success-Stories: Erfolgsbeispiele

Materialien 21: Vorträge: Vortragsfolien/Präsentationen

Materialien 22: Studie: KPMG - Wirtschaftpolitische Aktivitäten in Europäischen Regionen

Materialien 23: Informationsquellen: Informationsquellen \& Datenbanken

Materialien 24: Informationsquellen: Die fünf bedeutendsten Datenbanken des TecNet

Materialien 25: Expertengespräche: Evaluation und Expertengespräche

Materialien 26: Förderlinien: Adressen und Ansprechpartner von Förderinstitutionen

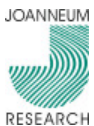

